\title{
Perspectives on the Role of Enzymatic Biocatalysis for the Degradation of Plastic PET
}

\author{
Rita P. Magalhães ${ }^{1,2}\left(\mathbb{D}\right.$, Jorge M. Cunha ${ }^{1,2}$ and Sérgio F. Sousa ${ }^{1,2, * \mathbb{D}}$ \\ 1 UCIBIO_Applied Molecular Biosciences Unit, BioSIM-Departamento de Biomedicina, Faculdade de \\ Medicina, Universidade do Porto, 4200-319 Porto, Portugal; ritaprata1@hotmail.com (R.P.M.); \\ jorgemcunha97@gmail.com (J.M.C.) \\ 2 Associate Laboratory i4HB-Institute for Health and Bioeconomy, Faculdade de Medicina, Universidade do \\ Porto, 4200-319 Porto, Portugal \\ * Correspondence: sergiofsousa@med.up.pt
}

Citation: Magalhães, R.P.; Cunha, J.M.; Sousa, S.F. Perspectives on the Role of Enzymatic Biocatalysis for the Degradation of Plastic PET. Int. J. Mol. Sci. 2021, 22, 11257. https://doi.org/ 10.3390/ijms222011257

Academic Editor: Sergio Marti

Received: 24 September 2021

Accepted: 16 October 2021

Published: 19 October 2021

Publisher's Note: MDPI stays neutral with regard to jurisdictional claims in published maps and institutional affiliations.

Copyright: (c) 2021 by the authors. Licensee MDPI, Basel, Switzerland. This article is an open access article distributed under the terms and conditions of the Creative Commons Attribution (CC BY) license (https:// creativecommons.org/licenses/by/ $4.0 /)$.

\begin{abstract}
Plastics are highly durable and widely used materials. Current methodologies of plastic degradation, elimination, and recycling are flawed. In recent years, biodegradation (the usage of microorganisms for material recycling) has grown as a valid alternative to previously used methods. The evolution of bioengineering techniques and the discovery of novel microorganisms and enzymes with degradation ability have been key. One of the most produced plastics is PET, a long chain polymer of terephthalic acid (TPA) and ethylene glycol (EG) repeating monomers. Many enzymes with PET degradation activity have been discovered, characterized, and engineered in the last few years. However, classification and integrated knowledge of these enzymes are not trivial. Therefore, in this work we present a summary of currently known PET degrading enzymes, focusing on their structural and activity characteristics, and summarizing engineering efforts to improve activity. Although several high potential enzymes have been discovered, further efforts to improve activity and thermal stability are necessary.
\end{abstract}

Keywords: PET; plastic; biodegradation; plastic degradation; PETase; MHETase; LCC; cutinase; Ideonella sakaiensis; biocatalysis

\section{Introduction}

Synthetic plastic materials are long-chain polymers with high durability and resistance, typically derived from fossil-fuel by-products [1]. Massive plastic production and usage began in 1950 [2,3], when 2 million tons were produced annually [4]. Production has tripled in the last 20 years [5]. According to a recent Plastics Europe [6] report, 359 million tons of plastic were produced worldwide in 2018. In 2019, this number grew to 368 million tons [6], and is expected to surpass 34 billion tons by 2050 [2], cumulative, of which 12 billion are expected to be deposited in landfills or contaminated natural environments [7]. The industries of packaging and construction amount to over $60 \%$ of plastic demand [6]. Most plastics produced are single-use, poorly discarded products, resulting in accumulation in various ecosystems [8] with serious consequences for soil, marine, and terrestrial species [9-12].

The most common strategy for plastic elimination is landfill deposition $[3,13]$. This is a hazardous strategy with countless disadvantages [14]. Degradation of plastic waste is slow, and its accumulation leads to environmental contamination of groundwater, marine, and terrestrial environments. Incineration is another harmful and widely used strategy, as it leads to the spreading of toxic and volatile wastes, which are dangerous to the environment and human beings [14,15].

Industrial plastic recycling is the conversion of plastic waste into reusable materials, typically by mechanical or chemical processing. However, currently employed tactics are not sufficient to satisfy the recycling needs of the ever-growing plastic industry [3]. 
Mechanical recycling does not change the chemical structure of the polymer, and implicates previously collecting, sorting, shredding, grinding, melting, and washing of the material [16-18]. These methods have several disadvantages-they are not applicable to temperature-sensitive or multilayer plastics [19], often result in material deterioration, and sorting brings in economical and applicability issues [20]. Furthermore, mechanical recycling is hazardous for the environment and constitutes a public health danger, since it is a common source of toxic organic compounds [21]. Chemical recycling typically leads to the polymer's degradation into its monomeric building blocks [18], via hydrolysis, methanolysis, and glycolysis strategies. However, it normally involves a high cost and high energy process, consuming active catalysts [22]. In addition, chemical methods are environmentally harmful, as they constitute a source of volatile organic compounds [23,24]. Biodegradation, the cleavage of plastic polymers into monomers by microorganism produced enzymes [25], is an environmentally friendly strategy [26] that has been gaining more attention. These microorganisms can use degradation products as a carbon or energy source through excretion of extracellular enzymes and metabolites [27-29]. These enzymes typically degrade the polymers with the assistance of water molecules and are known as hydrolases $[30,31]$. The by-products of enzymatic processing can be used for different applications, besides being naturally up taken by cells [13,24]. Spontaneous biodegradation is often preceded by oxidizing agents or UV-light [32]. Recent advances have placed plastic biodegradation at the top of promising recycling strategies, even if several limitations still remain to be solved [33].

Polyethylene terephthalate (PET) is one of the most used and synthesized plastics [9]. PET, derived from crude-oil [34], is composed of repeating units of terephthalic acid (TPA) and ethylene glycol (EG) monomers [35], and exists as an amorphous as well as a semicrystalline synthetic polymer [36]. It is typically produced by condensation of TPA and EG or bis(2-hydroxyethyl)terephthalic acid (BHET) and EG [37]. The main components and degradation products of PET are represented in Figure 1. The physiochemical properties of PET result in diverse applications in the textile, packaging and bottle producing industries [9]. PET bottle production in 2016 was of 485 billion unities [6]. In 2021, the estimate of production increased to 583 billion bottles [35]. PET fibers are extremely attractive for their high durability, elasticity, strength, and resistance to chemicals [38]. Unfortunately, the same characteristics that make PET an attractive material also result in difficult degradation and consequent accumulation in the environment [39]. Several strategies have been described for PET mechanical [21,40] and chemical [23,24,41,42] recycling, but the high cost and environmental impact of these methods lead to an urgent need for the development of more effective and greener alternatives.

PET is a high molecular weight polyester $[43,44]$, which presents a stable backbone and crystallinity, which inhibits natural breakdown $[34,45]$. As a semi-crystalline polymer with a glass transition temperature $\left(\mathrm{T}_{\mathrm{g}}\right)$ of $76{ }^{\circ} \mathrm{C}$ [37], PET presents amorphous and crystalline regions [46]. The $\mathrm{T}_{\mathrm{g}}$ is the temperature at which a reversible transition between a more tightly packed crystalline state and a more rubber-like amorphous state happens [47]. When PET is mostly in an amorphous stage, the polymeric chain is more readily available for enzymatic mediated biodegradation [48]. This is due to the increased mobility of PET chains in an amorphous stage in comparison with the crystalline state [49].

PET is by far the most studied polymer in terms of biodegradation, and the one with the largest number of degrading enzymes identified and characterized [50]. The first enzyme with PETase-like activity, secreted by Thermobifida fusca, was described by Müller et al. [51] in 2005. Since then, several lipases, cutinases, and esterases have been characterized [52-58]. In 2016, Yoshida et al. [52] identified a bacterium named Ideonella sakaiensis that secretes a consortium of PET degrading enzymes-IsPETase and IsMHETase. The discovery of specific PET hydrolases reinforced the interest for microbial PET degradation and inspired the identification, characterization, and enhancement of multiple enzymes. 

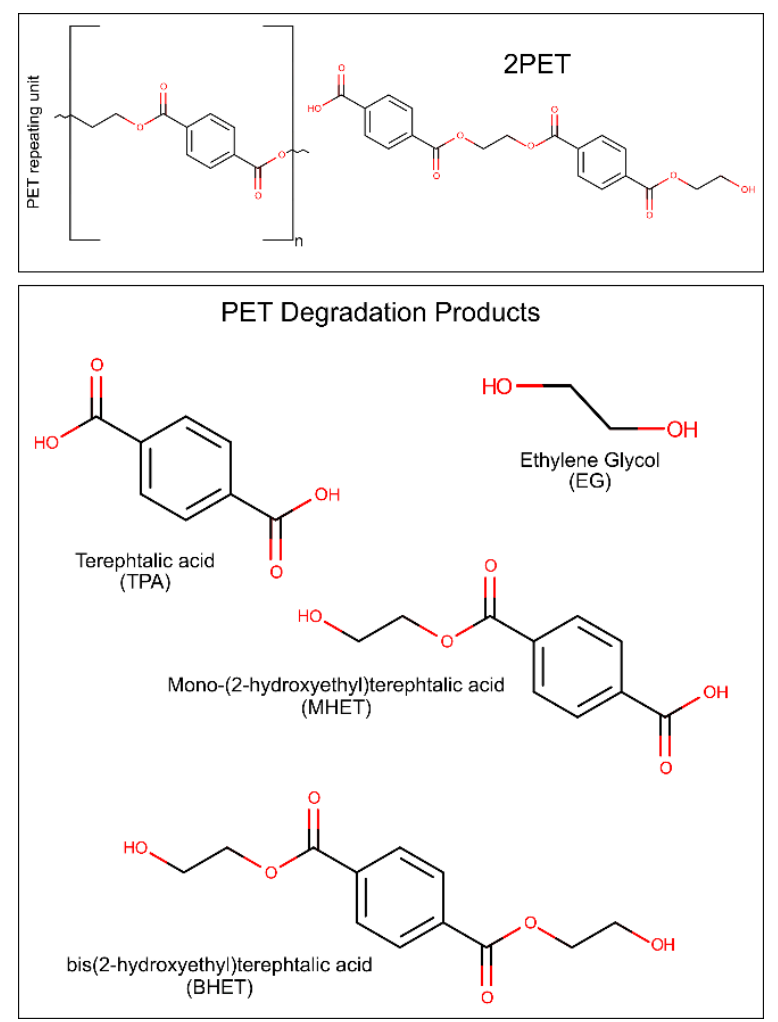

Figure 1. Scheme of the PET repeating unit, a molecule of 2PET, and the main PET degradation products.

The growing usage of enzymes in biodegradation is partially made possible by the evolution of diverse, but complementary, scientific fields. Even though enzymes are natural catalysts, their efficiency, stability, and catalytic turnover is often insufficient to be feasibly applicable [59]. Thus, diverse enzyme engineering approaches to improve catalytic, stability and productivity properties have been developed. Ultimately, the goal of enzymatic genetic engineering is to modify the amino acid sequences through gene alteration [60].

Some of the most employed genetic engineering methods are directed evolution, DNA shuffling, saturation mutagenesis, fusion, site-directed mutagenesis, and truncation [61]. In directed evolution, no data on the protein structure and function are required, and it works by building a library of randomly generated mutants. On the other hand, in rational enzymatic design or semi-rational design, information on protein structure and function is required $[60,61]$.

Enzymatic engineering through rational design, which implies introducing specific and pre-defined changes in the amino acid sequence by site-directed mutagenesis, has been growing as an emerging family of engineering techniques [60,62].

Since rational design implies knowledge of protein function and structure, it frequently begins with computer aided design [60]. The rapid increase of high-resolution protein structures available on the PDB database [63], and the improvement of structureprediction tools, such as the recently released AlphaFold [64], allows for molecular and atomistic perspectives on the structure and function of enzymes. This pipeline combines computational [65] and experimental [66] techniques to improve enzymatic thermal stability (through, for example, introduction of additional disulfide bonds), filling protein voids, increase enzymatic turnover and efficiency, or attribute new functions to the enzyme.

At this stage, several PET degrading enzymes have been discovered, and as a lot of work has been done on structural solving and characterization, the emerging trend for the future of the field is the engineering and improvement of these found enzymes. Structure resolution and atomistic understanding of an enzyme is tightly related with the ability to 
perform rational design, and several of the efforts employed on PET enzymes follow this line of thought, as mutagenesis is used both to confirm amino acid roles, and to improve enzymatic characteristics.

This review presents an overview of the current paradigm of PET enzymatic biodegradation in academic research, with a particular structural, mutagenic, and mechanistic perspective. Special attention is dedicated to the most promising PET degrading enzymes. In addition, we provide insight into several promising enzymes with PET degrading potential, which have been the subject of preliminary characterization in recent years, but that still require further biochemical and structural studies to confirm their full potential and explore/modulate their activity. Some microorganisms with the ability to utilize PET as an energy source are also highlighted. Finally, several enzymes with polyesterase activity but no specific PET activity are also discussed.

\section{Enzymes Involved in PET Degradation}

To date, more than 24 different enzymes with PET degrading ability have been identified. All of these enzymes are hydrolases, catalyzing the breaking of the PET polymer into TPA, EG, BHET, and (mono-(2-hydroxyehyl)terephthalic acid (MHET).

Hydrolases are enzymes that catalyze the breaking of chemical bonds through a reaction with water. They constitute a particularly versatile and ample class of enzymes, with an ability to act on diverse substrates of different sizes and complexity, such as proteins, carbohydrates, lipids, and nucleic acids. Hydrolases can be characterized by an enzyme commission number EC 3 and can be classified into 13 different subclasses, according to the specific types of bonds that they cleave [31]. In fact, this class of enzymes can act on extremely different bonds, with very different strengths [67]. The rates of uncatalyzed hydrolysis reactions of known substrates for hydrolases at $25^{\circ} \mathrm{C}$ have been shown to span over 17 orders of magnitude, with activation free energies in solution differing from 21 to $44 \mathrm{kcal} / \mathrm{mol}$ [68]. Hence, the challenge posed by nature to the different hydrolases can be quite diverse [31], and the intrinsic stability of PET, makes its enzymeassisted hydrolysis certainly a tough challenge. However, despite the challenge, different hydrolases present in different organisms have been shown to exhibit at least some PET degrading ability. For that, these enzymes employ a variety of strategies, some still not fully uncovered. Hydrolases involved in PET degradation act on ester bonds and are part of class 3.1 [69]. Furthermore, most PET degrading enzymes belong to the 3.40.50.1820 superfamily, according to the CATH database [70], since they share a conserved catalytic domain and assume the typical alpha/beta hydrolase fold. Breaking of PET bonds is typically accomplished by a catalytic triad involving a serine, a histidine and a negatively charged residue, usually an aspartate or a glutamate [71]. During the reaction, the product is commonly stabilized by an oxyanion hole made up of two or three residues [72].

The fact that PET exhibits a glass temperature $\left(\mathrm{T}_{\mathrm{g}}\right)$ of $76^{\circ} \mathrm{C}$, which is important to make the polymeric chain more accessible for enzymatic mediated biodegradation, constitutes an additional challenge for PET degrading enzymes, as such, enzymes must also be able to function at such high temperatures. Enzymatic melting temperature $\left(T_{m}\right)$ is the point when there is an equilibrium between protein folding and unfolding and is strongly associated with stability and catalytic ability [73]. As evidenced by Figure 2, most PET degrading enzymes exhibit $T_{m}$ lower than the $T_{g}$ of PET, even after engineering efforts. While enzymatic thermal stability is a complex problem, PET degrading enzymes use disulfide bonds as a stability strategy. Joo et al. [74] proposed a classification system for PET degrading enzymes that divides them in two major groups: enzymes with one disulfide bond belong to Type I, and enzymes with an additional bridge are Type II molecules. The second group is further divided into Type IIa and IIb depending on the specific amino acid composition of the enzymatic binding site. We followed this classification system when enough information on the enzyme and organism of origin was available. 


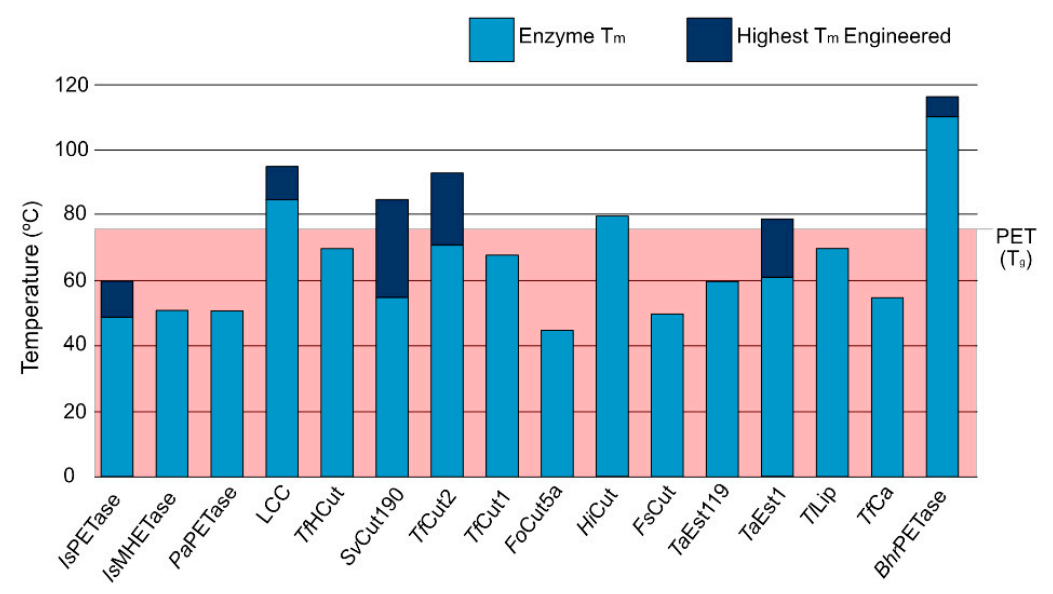

Figure 2. Melting temperature values for PET degrading enzymes and the highest Tm obtained through various engineering efforts in comparison with the glass temperature of PET.

The graphical scheme in Figure 3 summarizes the general information known on the main PET degrading enzymes, described in detail in the following sections. Figure 4 presents a structural representation of the most important PET degrading enzymes currently known, illustrating some of the features that characterize these enzymes, such as their catalytic triads, oxyanion hole residues, and presence of disulfide bonds. These characteristics are further explored throughout this review.

\begin{tabular}{|c|c|c|c|c|c|}
\hline Enzyme & Organism & $\begin{array}{l}\text { UniProt } \\
\text { Code }\end{array}$ & $\begin{array}{l}\text { Preferred } \\
\text { Substrates }\end{array}$ & $\begin{array}{c}\text { Major } \\
\text { Products }\end{array}$ & $\begin{array}{c}\text { Number of } \\
\text { Disulfide Bonds }\end{array}$ \\
\hline IsPETase & Ideonella sakaiensis & A0A0K8P6T7 & $\mathbf{0}$ & $\Delta$ & 2 \\
\hline IsMHETase & Ideonella sakaiensis & A0A0K8P8E7 & $\Delta$ & $\mathbf{\square}$ & 5 \\
\hline PaPETase & Pseudomonas aestusnigri & A0A0K8P6T7 & $\mathbf{0}$ & $\Delta$ & 2 \\
\hline LCC & unknown & G9BY57 & $\mathbf{0}$ & $\Delta \mathbf{E}$ & 1 \\
\hline TfHCut & Thermomonospora fusca & Q6A014 & C & $\mathbf{\square}$ & 1 \\
\hline SvCut190 & Saccharomonospora viridis & WOTJ6 & $\mathbf{0}$ & $\Delta \mathbf{\Delta}$ & 1 \\
\hline TfCut2 & Thermobifida fusca & E5BBQ3 & $\mathbf{0}$ & $\Delta \mathbf{\square}$ & 1 \\
\hline TcCut2 & Thermobifida cellulosilytica & E5BBQ3 & $\mathbf{0}$ & $\Delta \mathbf{\square}$ & 1 \\
\hline TcCut1 & Thermobifida cellulosilytica & E9LVH8 & C & $\Delta \mathbf{D}$ & 1 \\
\hline FoCut5a & Fusarium oxysporum & ХOBTD8 & $\mathbf{0}$ & $\Delta \mathbf{E}$ & 1 \\
\hline HiCut & Humicola insolens & A0A075B5G4 & $\mathbf{0}$ & $\Delta \mathbf{\Delta}$ & 1 \\
\hline FsCut & Thermomonospora fusca & Q6A014 & $\mathbf{0}$ & $\Delta \mathbf{\Delta}$ & 1 \\
\hline CaLipB & Candida antarctica & P41365 & $\mathbf{0}$ & $\mathbf{\Delta}$ & 3 \\
\hline TaEst1 & Thermobifida alba & D4Q9N1 & C & $\mathbf{\square}$ & \\
\hline Thip & Thermomyces lanuginosus & M4TP71 & $\mathbf{0}$ & $\Delta \mathbf{\Delta}$ & 3 \\
\hline TfCa & Thermobifida fusca & P86325 & $\mathbf{0}$ & $\mathbf{A}$ & 1 \\
\hline EsEstB & Enterobacter sp. HY1 & A0A4Y5UXE5 & $\mathbf{0}$ & $\Delta \mathbf{\Delta}$ & \\
\hline BhrPETase & HR29 & A0A2H5Z9R5 & $\mathbf{0}$ & $\Delta \mathbf{\Delta}$ & 1 \\
\hline BsEstB & Bacillus subtilis & A5HLW9 & $\mathbf{0}$ & $\Delta \mathbf{E}$ & \\
\hline ScSub1 & Streptomyces scabies & & $\mathbf{0}$ & $\mathbf{\square}$ & 2 \\
\hline PmCut & Pseudomonas mendocina & A4Y035 & C & & \\
\hline TcCut0390 & Thermomonospora curvata & D1A2H1 & $\mathbf{0}$ & & \\
\hline TcCut1278 & Thermomonospora curvata & D1A9G5 & $\mathbf{0}$ & & \\
\hline ThEst & Thermobifida alba & H6WX58 & 0 & $\Delta \mathbf{\square}$ & \\
\hline (1) PET & MHET $\square$ TPA $\triangle$ B & BHET $\oslash \mathrm{EG}$ & & & \\
\hline
\end{tabular}

Figure 3. General scheme summarizing the main PET degrading enzymes, their organisms of origin, UniProt Code, confirmed substrates and major products, and number of disulfide bonds. 


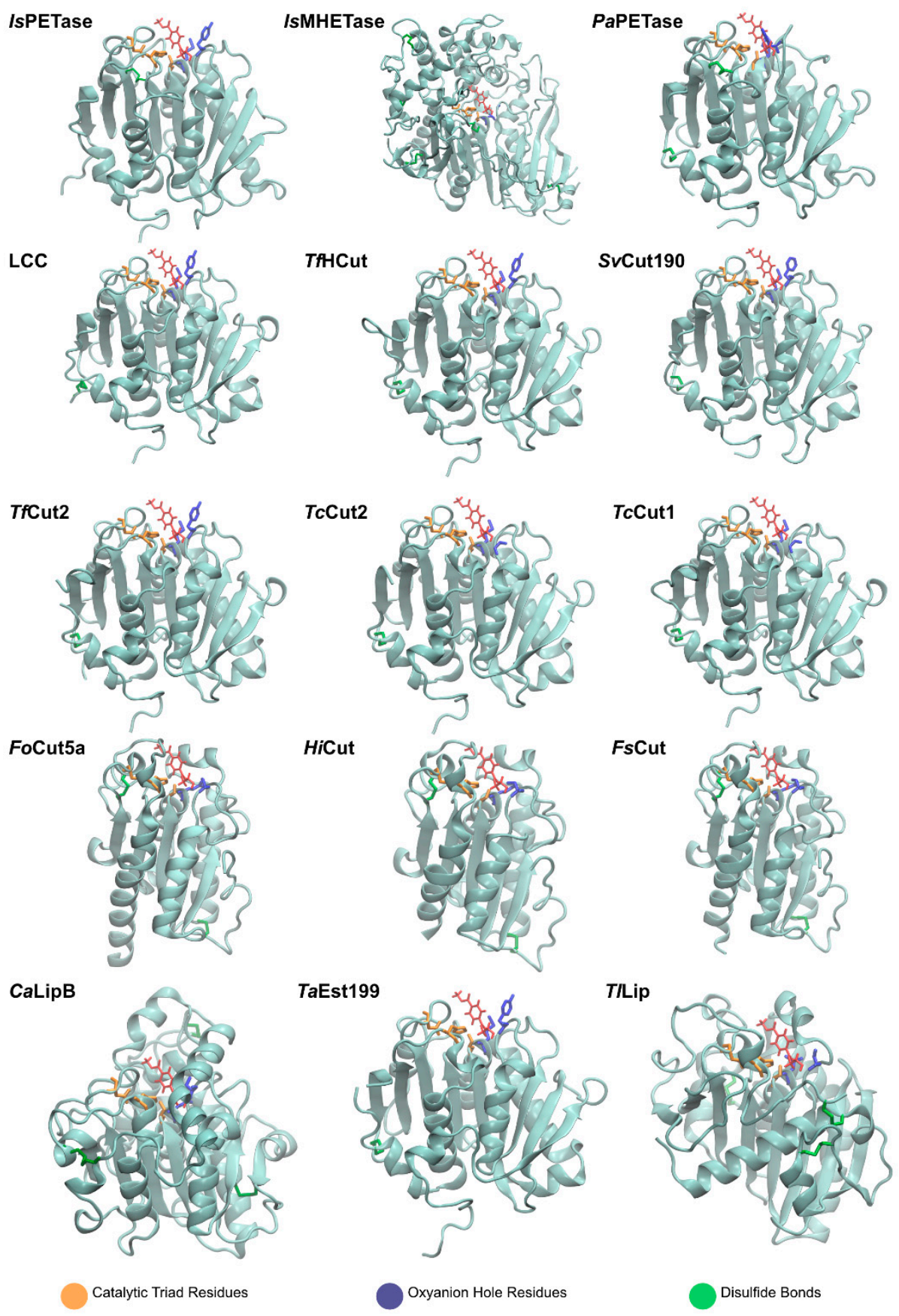

Figure 4. Main PET degrading enzymes known.

2.1. Ideonella sakaiensis PETase (IsPETase)

2.1.1. Discovery

IsPETase is a PET hydrolyzing enzyme first identified by Yoshida et al. [52] in 2016. This enzyme is responsible for degrading PET to MHET as a major product and EG, TPA, and BHET as secondary products [52,75]. The IsPETase producing bacterium, Ideonella sakaiensis 201-F6, is capable of assimilating PET as a major energy and carbon source. It was identified from a novel microbial consortium formed on PET film, isolated, and characterized as depending nutritionally on PET. Once it was confirmed that I. sakaiensis hydrolyzed PET to MHET, TPA, and BHET, two enzymes were identified as responsible, IsPETase and IsMHETase. IsPETase was determined to have higher activity against PET film and BHET than the previously identified enzymes TfHCut, LCC and FsCut by 120, 5.5 and 88 times 
(respectively) at $30^{\circ} \mathrm{C}$ and $\mathrm{pH}$ 7. However, activity was lower against aliphatic esters when compared with the mentioned enzymes, indicating IsPETase preference for PET [52].

\subsubsection{Structure}

IsPETase is composed of 290 amino acid residues, exists as a functional monomer [74], and its three-dimensional structure has been explored by several structural, experimental, and computational studies [74-76]. The enzyme presents a canonical $\alpha / \beta$-hydrolase fold with nine mixed $\beta$-strands that make up a central $\beta$-sheet, surrounded by seven $\alpha$ helixes [74]. At the active site, a conserved catalytic triad composed of a Serine (Ser160), Histidine (His237) and Aspartate (Asp206) residues is found in a broad active site cleft. Ser160 is inserted in a nucleophilic elbow with a sharp turn conformation [75] at the beginning of $\alpha 4$ [77]. The catalytic Asp206 residue is in the loop region between $\beta 7$ and $\alpha 5$, while His237 is found in the loop region between $\beta 8$ and $\alpha 6$ [77].

IsPETase presents a highly polarized surface charge, creating a dipole across the macromolecule and resulting in an isoelectric point of 9.6 [75]. Several PET degrading enzymes present charged surfaces, as evidenced by Figure 5 .

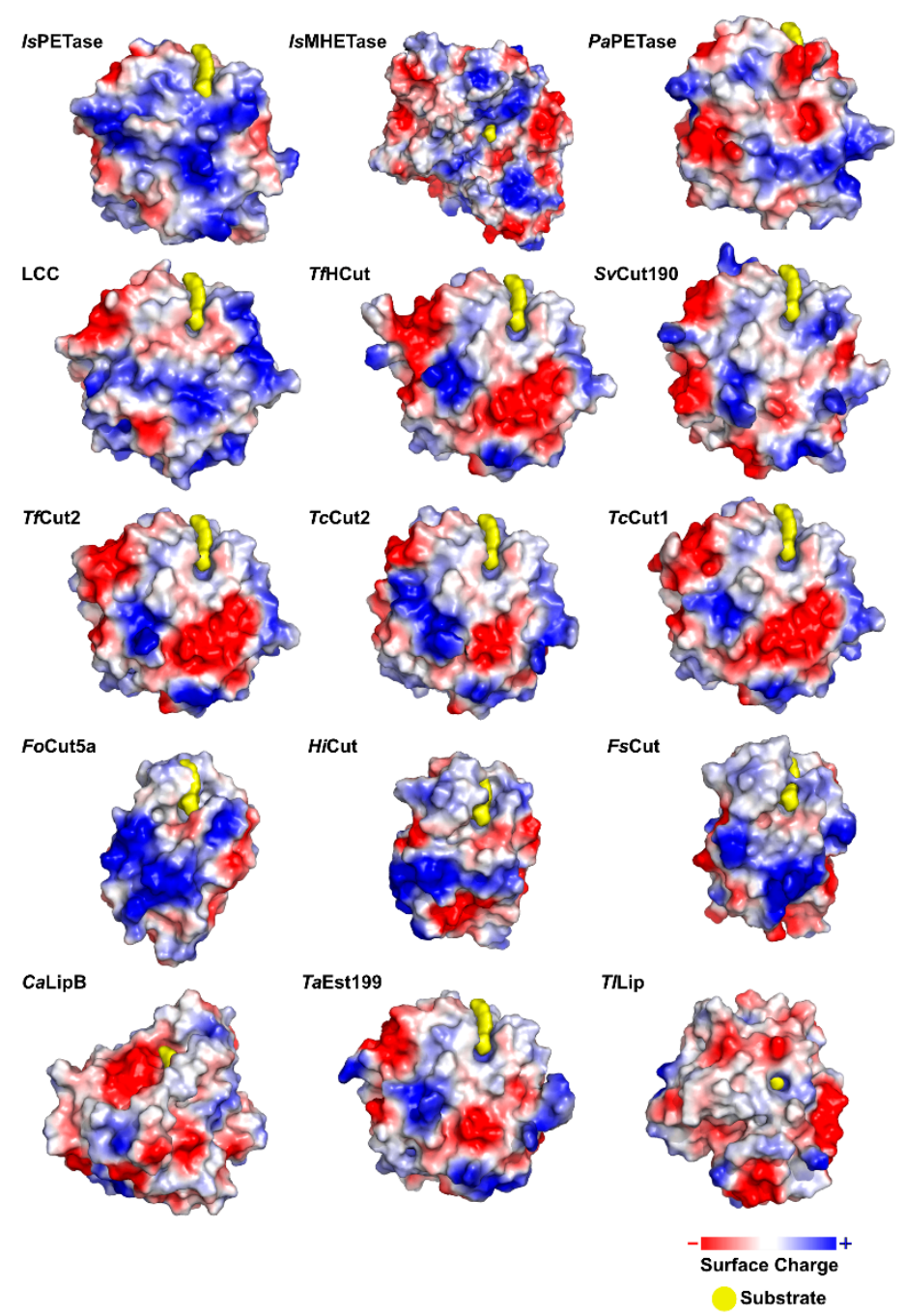

Figure 5. Graphical representations of the charged surfaces of the most important PET degrading enzymes.

The enzyme presents two disulfide bridges: DS1 (Cys203-Cys239) and DS2 (Cys273Cys289). DS2 is conserved amongst all known PET hydrolyzing enzymes, located near the C-terminal and distant from the catalytic center [74]. This bond does not directly intervene 
or contribute to hydrolysis, but has a critical role in structural integrity of the enzyme. DS1 is specific to IsPETase. Located near the active center, it has an essential role in catalytic activity and active site integrity. In other known PET hydrolases, this bond corresponds to highly conserved alanine residues [76]. In IsPETase, the bridge connects $\beta$-strand 7 to a loop connecting $\beta$-strand 8 and helix $\alpha 5$. This loop, anchored to the DS1 bond, contains the histidine catalytic residue. For its specificity and location, DS1 is thought to be one of the structural motifs responsible for the increased IsPETase activity when compared to other PET degrading enzymes [76,78].

A conserved Tryptophan residue (Trp185) assumes several conformations, termed A, B, and C, and is often referred to as the wobbly tryptophan, as represented in Figure 6. Even though the presence of this amino acid residue has been observed before in similar enzymes, its different conformations only appear in IsPETase, since in other known structures it always assumes the $\mathrm{C}$ conformation [76]. The flexibility of Trp185 was further confirmed by induced fit Docking and molecular dynamics studies [75]. In its vicinity is a serine (Ser214) residue (histidine in homologous enzymes), which allows for the A and B Trp185 conformers to be accommodated in the catalytic site [76].

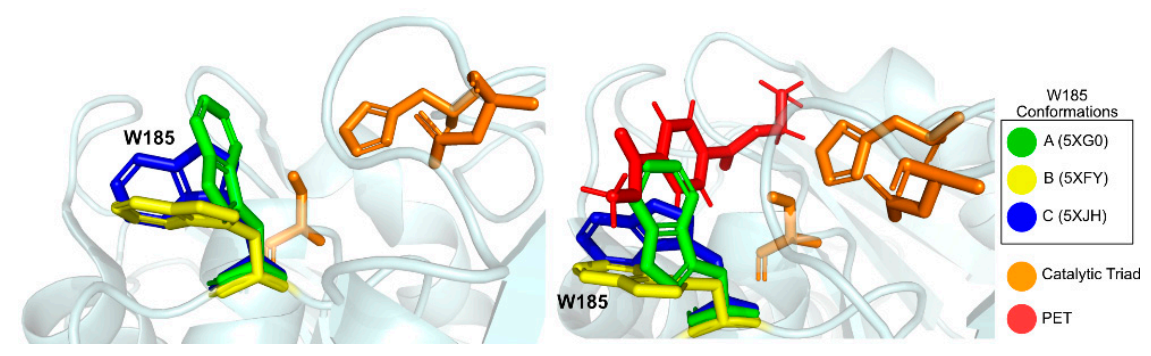

Figure 6. Representation of several conformations of residue Trp185, the wobbly tryptophan. Catalytic triad is represented in orange. Conformation A (PDB: 5XG0) is represented in green; conformation B (PDB: 5XFY) is represented in yellow; conformation C (PDB: 5XJH) is represented in blue. In the right side figure, HEMT, a PET model molecule (PDB: $5 \mathrm{XH} 3$ ), is represented, evidencing the optimal position of Trp185 in position C for catalysis.

Currently, there are 25 IsPETase structures available in the Protein Data Bank [63]. The structures, available in apo and complexed, mutated, and wild type form, are summarized and characterized in Table 1.

Table 1. Crystallographic structures of IsPETase.

\begin{tabular}{|c|c|c|c|c|c|}
\hline PDB Code & Resolution (Å) & Ligand & Mutations & Year of Deposition & Ref. \\
\hline $5 \times G 0$ & 1.58 & Free & - & 2017 & [76] \\
\hline $5 X F Y$ & 1.40 & Free & S131A & 2017 & [76] \\
\hline 5XFZ & 1.55 & Free & R103G/S131A & 2017 & [76] \\
\hline $5 \mathrm{ZH} 3$ & 1.30 & HEMT & R103G/S131A & 2017 & [76] \\
\hline $5 \mathrm{XH} 2$ & 1.20 & $p \mathrm{NP}$ & R103G/S131A & 2017 & [76] \\
\hline $5 \mathrm{XJH}$ & 1.54 & Free & - & 2017 & [74] \\
\hline 5YNS & 1.36 & Free & R280A & 2017 & [74] \\
\hline 6EQD & 1.70 & Free & - & 2017 & {$[75]$} \\
\hline $6 \mathrm{EQH}$ & 1.58 & Free & - & 2017 & [75] \\
\hline $6 \mathrm{EQG}$ & 1.799 & Free & - & 2017 & [75] \\
\hline 6EQF & 1.70 & Free & - & 2017 & [75] \\
\hline
\end{tabular}


Table 1. Cont.

\begin{tabular}{|c|c|c|c|c|c|}
\hline PDB Code & Resolution (Å) & Ligand & Mutations & Year of Deposition & Ref. \\
\hline 6EQE & 0.92 & Free & - & 2017 & [75] \\
\hline 6ANE & 2.02 & Free & - & 2017 & [79] \\
\hline $5 \mathrm{YFE}$ & 1.39 & Free & - & 2017 & [78] \\
\hline 6ILW & 1.575 & Free & - & 2018 & [34] \\
\hline 6ILX & 1.45 & Free & W159F & 2018 & {$[34]$} \\
\hline 6QGC & 2.0 & Free & - & 2019 & [80] \\
\hline $6 \mathrm{IJ} 3$ & 1.40 & Free & S121D/D186H & 2019 & [81] \\
\hline $6 \mathrm{IJ} 4$ & 1.86 & Free & S121D/D186H & 2019 & [81] \\
\hline $6 \mathrm{IJ} 5$ & 1.72 & Free & P181A & 2019 & [81] \\
\hline $6 \mathrm{IJ} 6$ & 1.95 & Free & S121E/D186H/R280A & 2019 & [81] \\
\hline $6 \mathrm{KY} 5$ & 1.63 & Free & $\begin{array}{l}\text { S214H/I168R/W159H/S188Q/R280A/ } \\
\text { A180I/G165A/Q119Y/L117F/T140D }\end{array}$ & 2019 & [82] \\
\hline $6 \mathrm{KUO}$ & 1.90 & Free & N246D & 2019 & [83] \\
\hline 6KUQ & 1.91 & Free & $\mathrm{A} 248 \mathrm{D} / \mathrm{R} 280 \mathrm{~K}$ & 2019 & [83] \\
\hline $6 \mathrm{KUS}$ & 2.00 & Free & S121E/D186H/S242T/N246D & 2019 & [83] \\
\hline
\end{tabular}

The first IsPETase structures were determined by Han et al. [76] in 2017. The first apostructure of IsPETase was solved at $1.58 \AA$ (PDB: 5XG0) and revealed the main structural findings already described. Despite several efforts, the authors were unable to obtain co-crystallized structures between wild type (WT) IsPETase and different ligands. For that reason, an inactive S160A (catalytic serine) and R159G double mutant was produced, and complexed structures were obtained with substrate (PDB: 5XH3) and product (PDB: 5XH2) analogues. Mutated apo-form enzyme structures were also resolved and used as a control to verify that the overall enzymatic fold and structure were unaffected by the point mutations necessary for obtaining the complexes.

Joo et al. [74] have solved and published several IsPETase structures since 2017 [81,83]. The group's apo-form enzyme was solved at $1.54 \AA$ (PDB: $5 \mathrm{XJH}$ ) and was used to perform molecular docking studies with 2-HE(MHET)4, a 4-MHET molecule mimicking a 4-moieties PET chain [74]. The binding mode of the first moiety at the active site confirmed the presence of the conserved catalytic triad (Ser160, His237, and Asp206) and of an oxyanion hole (Tyr87 and Met161) at appropriate distances for stabilization of the reaction intermediates. The docking of a longer molecule led to the conclusion that the binding site is a mostly hydrophobic, with a long and shallow L-shaped cleft on a flat surface. Furthermore, the binding site is divided into two subsites - subsite I (defined by residues Tyr87, Gln19, Met161, and Trp185) and II (residues Thr88, Ala89, Trp159, Ser238, and Asn241), being that subsite II is further partitioned into subsite IIa, IIb, and IIc. One PET moiety binds the catalytic center in subsite I, while subsite II accommodates the remaining three moieties through mostly hydrophobic interactions. The location of the subsites in relation to the catalytic triad and the substrate are represented in Figure 7 . The role of Trp185 in subsite I was evidenced by the $\pi-\pi$ interactions between this residue and the benzene ring in PET. This stabilization of the first PET moiety is aided by Met161 and Ile208 [74]. 


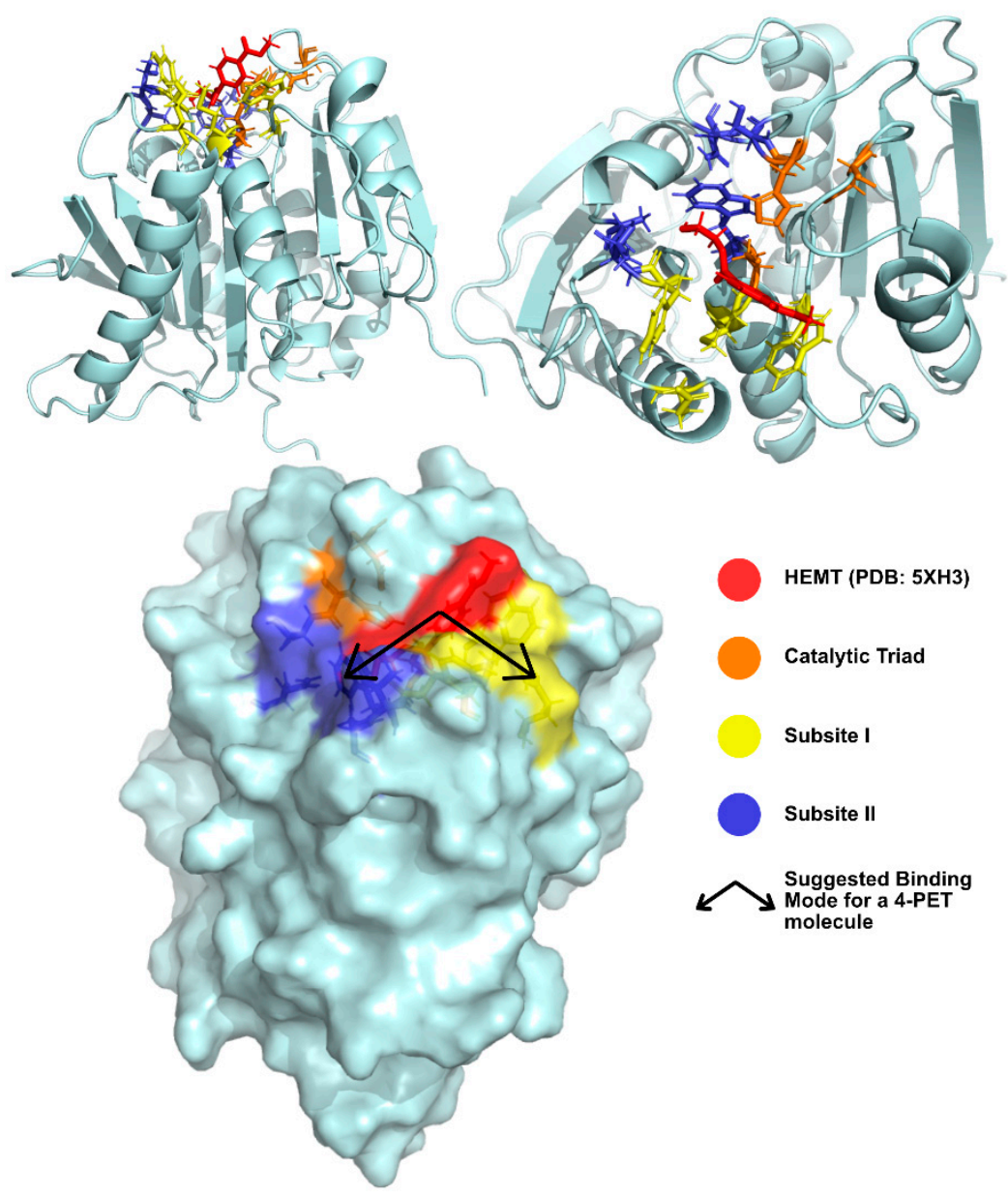

Figure 7. Binding Pocket of IsPETase suggested by Joo et al. [74] from different perspectives. HEMT, a PET model molecule, is represented in red. The catalytic triad is represented in orange. Binding subsites I and II are represented in yellow and blue, respectively.

\subsubsection{Activity}

Several mutagenesis and activity studies confirmed the catalytic and active role of many of the amino acid residues on the structural aspects proposed. IsPETase engineering assays published to date are summarized in Table S1.

Given the $\mathrm{T}_{\mathrm{g}}$ of PET, the polymer is more readily available for enzymatic degradation at higher temperatures [84]. However, the melting temperature $\left(\mathrm{T}_{\mathrm{m}}\right)$ of IsPETase was determined at $48.81{ }^{\circ} \mathrm{C}$ [81]. For that reason, engineering of PETase to increase enzymatic activity should be paired with engineering efforts to raise thermal stability and durability [77].

Mutagenesis of the catalytic triad to alanine residues (S160A, H237A, and D206A) consistently resulted in total loss of enzymatic activity, confirming their critical essential role as the catalytic triad [74,78]. Disulfide bridge DS1 disrupting mutations (C203S and C239S) resulted in very low enzymatic activity and lowered $\mathrm{T}_{\mathrm{m}}$ by over $10^{\circ} \mathrm{C}$, confirming the essential role of the IsPETase specific bridge in both activity and stability [74,76]. Most studies targeting the oxyanion hole and substrate interacting residue Tyr87 with various substrates (PET film, PET bottle and BHET monomers) reported diminished activity and lower amounts of product released $[74,76,83]$, although in one study, a slight activity increase is reported [78]. Engineering of the other oxyanion hole amino acid residue Met161 consistently resulted in lower PETase activity against all tested substrates $[74,76,78,83]$. The wobbling Trp185 has also been the target of engineering studies-replacement of this residue with an alanine results in highly diminished to total loss of activity [74-76,78]. Residue Ser214, thought to influence Trp185 flexibility, has been engineered to a histidine residue (similar to other enzymes with PET hydrolytic activity), in two different studies. Results were 
non-consensual, since the mutation has both led to partly compromised activity [76] and slightly increased activity [78], depending on the study.

Several studies focused on engineering substrate binding residues in an attempt to increase enzymatic degradation activity. Han et al. [76] mutated Ile208, Trp159, Thr88 to alanine residues, with consistent decreases in PETase activity, showing the importance of these residues in catalysis. These findings were also corroborated by Joo et al. [74].

In addition, Joo et al. [74] explored residues from the binding subsite II (W159A, S238A, N241A) that resulted in activity reductions. To simulate TfCut2 binding site, Ser238 was mutated to phenylalanine (S238F) and Trp159 to histidine (W159H), with drastic consequences to IsPETase hydrolytic activity. The observation that Arg280 (located in subsite IIc) presented a protruding shape that seemed to destabilize the binding of PET led the authors to produce a R280A variant. This mutant presented augmented PET degrading activity against PET film by $22.4 \%$ in $18 \mathrm{~h}$ and $32.4 \%$ in $36 \mathrm{~h}$ when compared with the WT enzyme. The resolved structure (PDB: 5YNS) showed that alanine provided a hydrophobic and non-protruding cleft, resulting in an extended subsite IIc. Interestingly, even though Arg280 is $\sim 23 \AA$ away from the catalytic center, this mutation highly affected PETase activity, since it resulted in structural changes that led to better substrate accommodation. This observation suggests that key structural changes away from the active site have the potential to impact enzymatic activity. This finding inspired the authors in subsequent mutagenic studies [81,83].

In 2019 [81], the group published a manuscript applying the same rational protein engineering strategies to explore different mutations, using PET film as a substrate. Residue Pro181 disrupts the enzyme's secondary structure, suggesting a negative effect on enzymatic thermal stability and catalytic efficiency. Therefore, the variant P181A was produced, and resulted in diminished activity at both $30^{\circ} \mathrm{C}$ and $40^{\circ} \mathrm{C}$, besides having a higher $\mathrm{T}_{\mathrm{m}}$ value than WT IsPETase by $0.5^{\circ} \mathrm{C}$. After resolving and inspecting the P181A IsPETase structure (PDB: 6IJ5), it was verified that the mutation led to a collapse of the catalytic site since it shifted catalytic Asp206 away from the His237 residue. The authors, inspired by the structure of TfCut2, an enzyme with higher thermal stability than PET, designed a S121D/D186H double mutant to increase the stability of the $\beta 6-\beta 7$ connecting loop since these residues were thought to form an additional hydrogen bond. Indeed, the $\mathrm{T}_{\mathrm{m}}$ of this variant was of $54.85^{\circ} \mathrm{C}, 6^{\circ} \mathrm{C}$ higher than the $T_{m}$ of WT enzyme. This mutation resulted in an activity increase of 2.3- and 2.0-fold after 24 and $72 \mathrm{~h}$, respectively, at $30^{\circ} \mathrm{C}$, and a 3.4- and 4.4 -fold after 24 and $72 \mathrm{~h}$, respectively, at $40^{\circ} \mathrm{C}$. The effect on enzymatic activity is likely due to the increased thermal stability, which allows the enzyme to remain active longer at higher temperatures. IsPETaseS121D/D186H, inspired by these findings, was designed to be even more stable-which proved to be true, since this variant had a measured $\mathrm{T}_{\mathrm{m}}$ of $56.02{ }^{\circ} \mathrm{C}$ and an increased enzymatic activity of 2.2- and 2.6-fold after 24 and $74 \mathrm{~h}$, respectively, at $30^{\circ} \mathrm{C}$, and 4.7 - and 6.0 -fold at 24 and $72 \mathrm{~h}$, respectively, at $40^{\circ} \mathrm{C}$. Even though these variants increased PETase activity and stability by a fair amount, the authors combined these findings with the previous ones and designed two triple mutants-S121D/D186H/R280A and S121E/D186H/R280A. The measured $\mathrm{T}_{\mathrm{m}}$ for these variants were of 56.41 and $57.62{ }^{\circ} \mathrm{C}$, 7.6 and $8.81{ }^{\circ} \mathrm{C}$ higher than WT IsPETase. The activity of these variants was also higher than those of previously reported enzymes. S121E/D186H/R280A showed a 4.3 and 5.2 higher fold enzymatic activity after 24 and $72 \mathrm{~h}$, respectively, at $30{ }^{\circ} \mathrm{C}$, and a 9.1 and 13.9 higher fold after 24 and $72 \mathrm{~h}$, respectively, at $40{ }^{\circ} \mathrm{C}$.

Recently, the same group published a third mutagenic study on IsPETase [83]. After performing molecular docking experiments with a 2-HE(MEHT) ${ }_{4}$ molecule, several point mutations on substrate binding residues were designed, inspired by conserved and observed residues in other PET hydrolytic enzyme candidates. Most mutations led to slightly (Y87F, I208V, S238T) or drastic (T88L, R90S, I208T, G234N) activity decreases. However, mutations S242T and N246D resulted in significant activity increase. For that reason, efforts to incorporate these mutations into the previously designed triple mutants were developed and a S121/D186H/N246D/280A variant was designed. Unfortunately, this mutated 
IsPETase exhibited a decrease in activity and thermal stability when compared with the previously reported variant. After structural studies, it was discovered that the R280A and N246D mutations were not compatible and could not be employed simultaneously. Therefore, triple mutants incorporating each of these variants were designed, and the results revealed that $\mathrm{N} 246 \mathrm{D}$ was the more beneficial mutation for enzymatic activity and thermal stability. For that reason, a quadruple S121E/D186H/S242T/N246D mutant was produced (PDB: 6KUS). Both the $\mathrm{T}_{\mathrm{m}}$ and hydrolytic activity of these variant were higher than those of previous enzymes. This variant showed a gradual increase of PETase degradation activity up to 20 days, meaning a 58 -fold higher activity than WT IsPETase at $37^{\circ} \mathrm{C}$. These results are remarkable and extremely promising - a true testament to the potential of rational protein design.

Austin et al. [75] produced a S238F/W159H double mutant with the goal of converting the IsPETase binding site into a cutinase-like active site cleft. IsPETaseS238F/W159H resulted in higher enzymatic activity (evidenced by an increase in product release) and crystallinity reduction against PET film when compared with WT enzyme. To understand the increase in activity, the authors performed induced fit docking and theorized that the introduced Phe238 performed stabilizing aromatic interactions with the substrate, as well as with other amino acid residues in the active site.

The best performing IsPETase variant published to date was developed by Cui et al. [82] through a novel computational strategy termed GRAPE (greedy accumulated strategy for protein engineering). GRAPE employs a strategy for optimization of mutations in a cluster manner, creating several functional variants and selecting the most promising ones [82]. It is a rational and efficient way to combat the tardy strategy of exploring point mutations individually and combining them in various manners, requiring experimental testing at each stage. Initially, the algorithm identified 21 stabilizing mutations; however, a variant containing all of these mutations proved to be inactive, indicating some of them were not compatible when employed simultaneously. Then, a clustering method (K-means algorithm) was used to generate promising combinations of mutations. Several variants were tested, but DuraPETase (S214H/I168R/W159H/S188Q/R280A/A180I/G165A/Q119Y/ L117F/T140D) was by far the most successful [82]. It had been shown that WT IsPETase suffers total loss of activity within $24 \mathrm{~h}$ at $37^{\circ} \mathrm{C}$ [81]—DuraPETase, having a much higher $\mathrm{T}_{\mathrm{m}}$ and, therefore, thermal stability, resulted in high activity for longer periods at higher temperatures. Besides exhibiting a 300-fold activity increase in 10 days incubation with crystalline PET at $37^{\circ} \mathrm{C}$, the variant was active and functional for up to 3 days incubation at $60{ }^{\circ} \mathrm{C}$ [82], which is a remarkable achievement. The variants' ability to degrade nano and microplastics, one of the major issues for marine environments [85], was also much higher than that shown by WT IsPETase. DuraPETase also presents less specificity than the WT enzyme and can degrade other plastics such as polybutylene terephthalate (PBT) and polyethylene 2,6-naphthalenedicarboxylate (PEN) [82]. The authors solved the three-dimensional structure of DuraPETase (PDB: 6KY5) at $1.63 \AA$ to understand the mechanistic and structural attributes responsible for the enhanced activity. The overall structure of the variant is similar to WT IsPETase, and so was the PET binding mode predicted through molecular docking studies. The features highlighted as responsible for the increased hydrolytic activity were novel electrostatic interactions (T140D, I168R, W159H, S188Q), improved hydrophobic packing (Q119Y, A180I, S214H, R280A, L117F), reduction of conformational entropy (G165A). L117F and Q119Y provided additional stabilization of the typical binding mode, similar to the role performed by Trp185, Met161, and Tyr87 in the WT enzyme [82].

Liu et al. [34] tested IsPETase enzymatic activity under different conditions. Threepoint mutations (S93M, W159F, and N241F) were shown to increase enzymatic degradability of $p$-nitrophenyl esters, contrary to the highly specific WT enzyme. These mutations change the hydrophobicity and reduce the steric effects responsible for enzymatic specificity. IsPETase typically performs best under mild temperature conditions $\left(35^{\circ} \mathrm{C}\right)$ and loses stability as the temperature increases, accompanied by a decrease in activity. Immobiliza- 
tion of the enzyme by ammonium sulfate precipitation and glutaraldehyde cross-linkage resulted in maximum activity from 35 to $45^{\circ} \mathrm{C}$ and $60 \%$ of activity at $65{ }^{\circ} \mathrm{C}$. Regarding physicochemical factors, IsPETase was most active in buffer containing $20 \%$ glycerol and its activity increased as the buffer salt concentration increased. Activity was proportional to salt concentration from 100 to $500 \mathrm{mM} . \mathrm{Na}_{2} \mathrm{SO}_{4}$ was the highest impacting salt on enzymatic activity. However, IsPETase was inhibited by organic solvents, protease inhibitors, and detergents typically used in the industry and laboratory, such as propanol, ethanol, SDS, Tween 20, PMSF, and Triton X-100. The activity increase under high salt concentrations was suggested to most likely be due to a potential cation binding-site and anion-binding site in the enzyme [56].

In order to enhance IsPETase activity and stability, Chen et al. [86] built a whole cell biocatalyst of PET functionalized on the surface of Pichia pastoris yeast cell. Assays with PET film for $18 \mathrm{~h}$ at $\mathrm{pH} 9$ and $30^{\circ} \mathrm{C}$ resulted in a 36-fold increase in activity when compared with individual IsPETase. Activity was determined by measuring the produced amounts of MHET product. Furthermore, the complex system performed at a stable turnover rate for seven repeated uses under the same conditions, and was further tested with commercial highly crystalline PET bottles, having shown degradation ability. This study presented a promising direction for IsPETase activity increase and wider industrial applicability.

Inspired by the advantages of using microalgae for expression and production of plastic degrading enzymes, Moog et al. [87] employed P. tricornutum for IsPETase production and Kim et al. [88] used C. reinhardtii microalgae. In both studies, IsPETase activity with morphological changes on PET substrate and production of PET degradation metabolites such as TPA was observed. These studies suggest that using microalgae for IsPETase expression and introduction in the environment is an attractive and promising strategy, especially given the natural characteristics of microalgae - their abundance and low maintenance growth in natural aqueous systems with no endotoxin production [88].

\subsubsection{Proposed Mechanism}

The structural and mutagenic findings have led to a catalytic mechanism proposal for PET degradation by IsPETase. It is consensual that the mechanism follows a classical serine hydrolase family mechanism, similar to cutinase [89]. The only difference seems to be the role of Trp185, the wobbly tryptophan near the catalytic center. The movement of this residue is thought to have an essential role in both substrate binding and product release [90]. The current main mechanistic proposals are presented by the same groups that resolved the first known IsPETase three-dimensional structures. While Han et al. [76] focused on a detailed proposal on the role of each amino acid residue in the catalytic triad and the movements of atoms and electrons, Joo et al. [74], inspired by their findings on the enzyme's binding site, proposed a broader mechanistic view, considering the several possible products of PET hydrolysis. Since both proposals fit together to yield a complete mechanism, they will be integrated and explained simultaneously in this work and represented in Figure 8. 


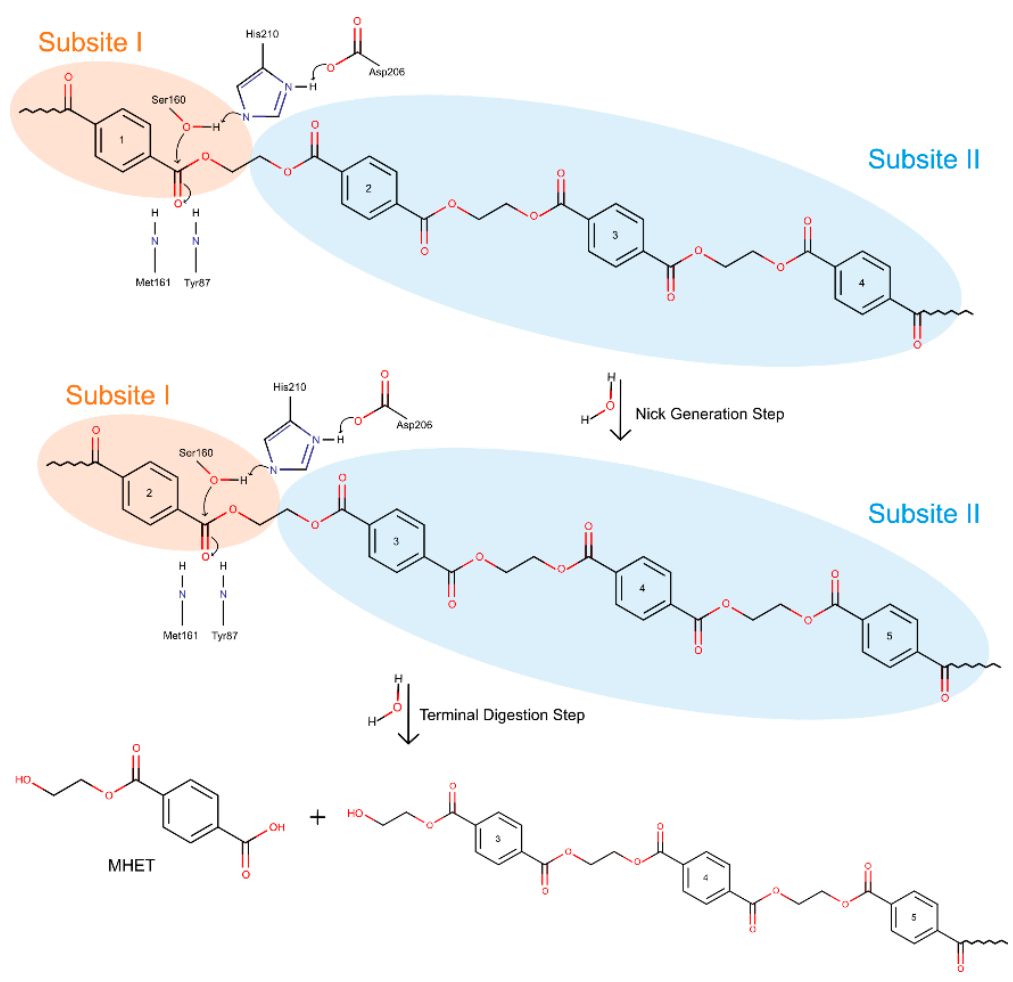

Figure 8. Scheme mechanism of IsPETase.

The presently accepted mechanism initiates with the binding of IsPETase to the substrate. The shallow cleft on apo-form enzyme allows for substrate binding onto the protein surface $[74,76]$. This is the beginning of the nick generation step, in which four moieties bind the protein —one to subsite I, where the catalytic reaction takes place, and the remaining three onto subsite II [74]. Upon binding, the cleavable ester bond is in optimal position to be attacked by Ser160 and stabilized by the oxyanion hole (Met161 and Tyr87) [76] - this cleavage leads to a nick in PET, resulting in two PET chains with distinct terminals released from each subsite: a TPA-terminal from subsite I and a hydroxyethyl terminal (HE terminal) from subsite II [74].

The considered mechanistic proposal suggests the first step to be a charge-relay system between catalytic residues Asp206 and His237. This system allows His237 to deprotonate the hydroxyl group on Ser160, which becomes a stronger nucleophile and attacks the carbonyl group of the PET ester [77]. The attack initiates catalysis and leads to the formation of the first tetrahedral intermediate, stabilized by the oxyanion hole. The intermediate's negative charge is highly unstable, and breaks down, resulting in the release of the first product and in an acyl-enzyme intermediate [76,77]. A second nucleophilic attack by a water molecule (deprotonated by the same His237-Asp206 charge-relay system) to the carbonyl carbon results in the second tetrahedral intermediate. Equally unstable, the intermediate breaks down and releases the second product. This step finishes with the full regeneration of the catalytic triad, so that another catalytic cycle can begin [76,77].

In the terminal digestion step, the two cleaved chains are digested into MHET (and BHET, TPA, and EG, in residual amounts) in different ways depending on the terminals of each molecule. For the HE terminal PET, once the four moieties were bound to subsite I and II, the already described ester bond breakage results in the production of a MHET monomer (first product released) and a HE-PET(n-1). The digestion of this HE-PET molecule follows the same steps as the first ester bond cleavage process [74]. The TPA-terminal PET molecule positions itself in the binding site with the TPA terminal at subsite I and the remaining three PET moieties in subsite II. In this case, the cleavage of the ester bond produces one TPA molecule (first product) and a HE-PET(n-1), which suffers a similar breakage as described previously [74]. The different bindings and terminals generated result in a 
series of PET monomers and dimers that are eventually digested to the final products in a combinatorial manner, leading to the accumulation of four molecules-MHET, TPA, EG, and BHET. Finally, BHET can be degraded into MHET and EG, and the final products of IsPETase activity are MHET, TPA, and EG [74].

The binding mode proposed by Joo et al. [74], of 4-MHET moieties binding IsPETase simultaneously, was recently contested by Wei et al. [91]. The authors performed NMR analysis of amorphous PET at $30^{\circ} \mathrm{C}$ and observed that the polymer does not acquire the conformation necessary to fit the docking predictions presented by Joo et al. [74] and, therefore, reject the binding mode and the overall mechanistic proposal based on it. Furthermore, Wei et al. [91] performed comparative activity assays with IsPETase and LCC at their respective optimal activity temperatures $\left(30^{\circ} \mathrm{C}\right.$ and $70^{\circ} \mathrm{C}$, respectively) for $24 \mathrm{~h}$ and observed a 40-fold higher weight loss of PET material with LCC when compared with IsPETase. This suggests LCC activity on PET is much higher than that of IsPETase, which is in disagreement with the original activity reports by Yoshida et al. [52]. Recently, Joo et al. [92] responded to Wei et al.'s [91] publication, arguing that the docking experiments did not consider temperature and, therefore, it is possible that the 4-MHET moiety polymer acquires the proposed conformation at higher temperatures. Moreover, the authors argued that even though IsPETase has a low thermal stability, several variants with higher melting temperatures have resulted in increased activity, which means that at higher temperatures the proposed binding mode and mechanism are plausible. Even though it is true that at higher temperatures amorphous PET content increases and, consequently, so does conformational freedom, it now seems plausible that the proposed binding mode and mechanism are neither likely nor favorable at a $30{ }^{\circ} \mathrm{C}$ temperature. This apparent low availability of PET chains at lower temperatures might also be the reason for the several reports on lower IsPETase activity than originally described by Yoshida et al. [52]. Similarly, Kawai et al. [93] questioned the classification of IsPETase as a PET degrading enzyme, arguing that PET degradation implies that the amorphous region of the polymer be attacked first, and only then the crystalline region becomes susceptible to hydrolysis. Therefore, since at $30{ }^{\circ} \mathrm{C}$ the amorphous content of PET polymer is residual, IsPETase would not be able to attack the polymer and degrade it in the described manner. Although further and more expansive studies on IsPETase activity on different PET substrates and at different temperatures are necessary, it seems too drastic to claim no PET hydrolytic activity can be attributed to IsPETase, given the exhaustive and multiple studies published, and hereby summarized on this enzyme. In conclusion, IsPETase activity, mechanism of action, and power is still not fully consensual, and future studies will be important to clarify some of the issues raised in recent years regarding IsPETase potential.

\subsubsection{Future Perspectives}

At this stage, knowledge about th IsPETase structure, function, and mechanism is quite extensive. Moreover, with the increasing need for PET degrading alternatives, new studies on this enzyme continue to appear. Several studies have resulted in X-ray structures of IsPETase with different variations, in terms of mutations and bound-molecules, and proposed what specific characteristics led to the augmented IsPETase enzyme when compared with other PET hydrolases. However, and even though several engineered IsPETase variants present higher $T_{m}$ values and preserve activity at higher temperatures, and for longer, these variants are still far from being usable in industrial contexts. Furthermore, the recent doubts cast on IsPETase activity and mechanism posed by Wei et al. [91] and Kawai et al. [93] highlight the need for additional analysis of this enzyme's activity and potential as an effective PET hydrolase. Even so, in line with the generally accepted activity reports, the strategies developed by Son et al. [83] and Cui et al. [82] constitute a promising methodology for the development of more active and durable plastic degrading enzymes. Furthermore, the non-engineering strategies explored by Liu et al. [34] represent an interesting preliminary evaluation of IsPETase as a broader plastic degrading enzyme and introduces valuable information on its performance in industrial settings. 


\subsection{Ideonella sakaiensis MHETase (IsMHETase)}

\subsubsection{Discovery}

IsMHETase is the second PET hydrolyzing enzyme identified in Ideonella sakaiensis 201-F6 by Yoshida et al. [52] in 2016. It is an intracellular enzyme with a molecular weight of $65 \mathrm{kDa}$ and 603 amino acids. According to ESTHER database, IsMHETase is labeled as a tannase that belongs in the $\alpha / \beta$-hydrolase Block $X$ family [94]. This superfamily is comprised not only by fungal and bacterial tannases, but also by feruloyl esterases. Its properties as a PET biocatalyst were firstly described by Yoshida et al. [52] in 2016, when it was shown to degrade MHET to EG and TPA, catalyzing the subsequent step of PET degradation initiated by IsPETase. Later studies confirmed that IsMHETase had no activity on BHET [77].

\subsubsection{Structure}

Studies on the IsMHETase structure show that it exists as a monomer [95], where the catalytic domain adopts an $\alpha / \beta$-hydrolase fold, similar to serine hydrolases, and the lid domain is larger than the average lid domain of $\alpha / \beta$-hydrolases. IsMHETase lid domain is composed of $\sim 240$ amino acid residues, while the average length in $\alpha / \beta$-hydrolases is about $\sim 100$ residues, as evidenced by Figure 9 . The lid domain partially involves the active site and a $\mathrm{Ca}^{2+}$ binding-site, similar to Aspergillus oryzae FaeB (AoFaeB), known to increase lid domain stability [96].

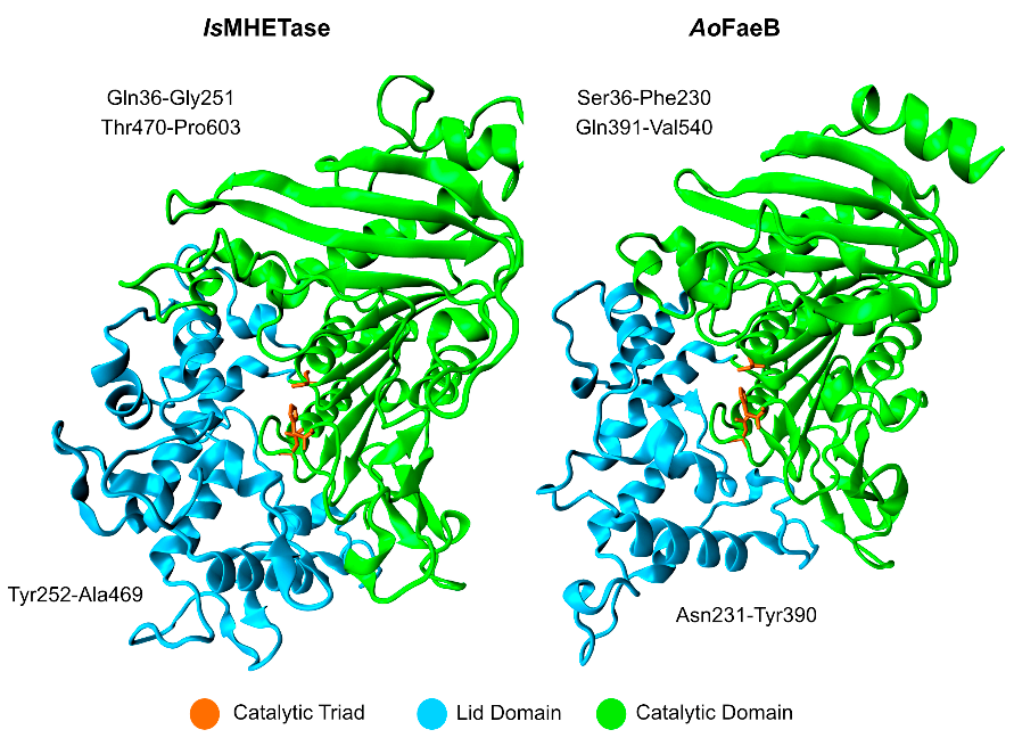

Figure 9. Lid and catalytic domains of IsMHETase in comparison with those of AoFaeB. The catalytic triad for each enzyme is represented in orange.

Contrary to IsPETase, IsMHETase has a more heterogenous and acidic surface, resulting in a lower isoelectric point (5.11) [89]. The enzyme contains five disulfide bonds (Cys51Cys92, Cys224-Cys529, Cys303-Cys320, Cys340-Cys348, and Cys577-Cys599), which are conserved in tannase family members [89]. The one binding Cys224 to Cys529 is in the active site and flanks the catalytic triad, which is formed by Ser225, His528, and Asp492, and the oxyanion hole, which is composed of the backbone amide nitrogen atoms of Gly132 and Glu226. Due to the properties of disulfide bonds, it is most likely that this bond tightens the catalytic triad, increasing IsMHETase stability.

Currently, there are nine three-dimensional structures of IsMHETase, including the WT enzyme in complexed and apo-form. These structures are summarized in Table 2. 
Table 2. Crystallographic structures of IsMHETase.

\begin{tabular}{cccccc}
\hline PDB Code & Resolution (̊̊) & Ligand & Mutations & Year of Deposition & Ref \\
\hline 6QGA & 2.1 & MHETA & - & 2019 & {$[80]$} \\
\hline 6QGB & 2.2 & Benzoic acid & - & 2019 & {$[80]$} \\
\hline 6QG9 & 2.05 & Free & - & 2019 & {$[80]$} \\
\hline GJTU & 2.1 & Free & - & 2019 & {$[95]$} \\
\hline 6JTT & 2.51 & BHET & - & 2019 & {$[95]$} \\
\hline 6QZ1 & 1.7 & Benzoic acid & - & 2019 & {$[89]$} \\
\hline 6QZ2 & 1.9 & Benzoic acid & - & 2019 & {$[89]$} \\
\hline 6QZ3 & 1.6 & Free & - & 2019 & {$[89]$} \\
\hline 6QZ4 & 1.8 & Free & - & 2019 & {$[89]$} \\
\hline
\end{tabular}

The first crystal structures of IsMHETase were determined in 2019 by Palm et al. [80]. The authors showed that IsMHETase binds to MHETA, a non-hydrolyzable substrate analogue of MHET (PDB: 6QGA), through hydrophobic contacts between the phenyl ring and $\alpha / \beta$-hydrolase residues Phe495, Gly132, and Ala494. In a similar way, the lid domain residues Phe415, Leu254, and Trp397 also establish hydrophobic contacts with the phenyl ring. Further studies confirmed the same binding mode with MHET [89,95]. Phe415 undergoes an induced-fit conformational change, pointing in the opposite way (open position) from the active site when the enzyme is in its free form; thus, promoting substrate binding and pointing towards the active site (closed position) when the enzyme is bound to a substrate. Arg411, which is sustained by Ser416, Ser419 and the backbone amide of Gly258, connects the two oxygens of the MHET free carboxylate.

Lastly, Knott et al. [89] solved four structures. Here, the group demonstrated that the carboxylate motif of MHET establishes hydrogen bonds with Arg411 and Ser416. Just like Phe 415, Gln410 also shows a concerted movement, where the side chain pivots towards the active site, when Phe415 is pointing in the opposite way from the active site, as can be seen in Figure 10.

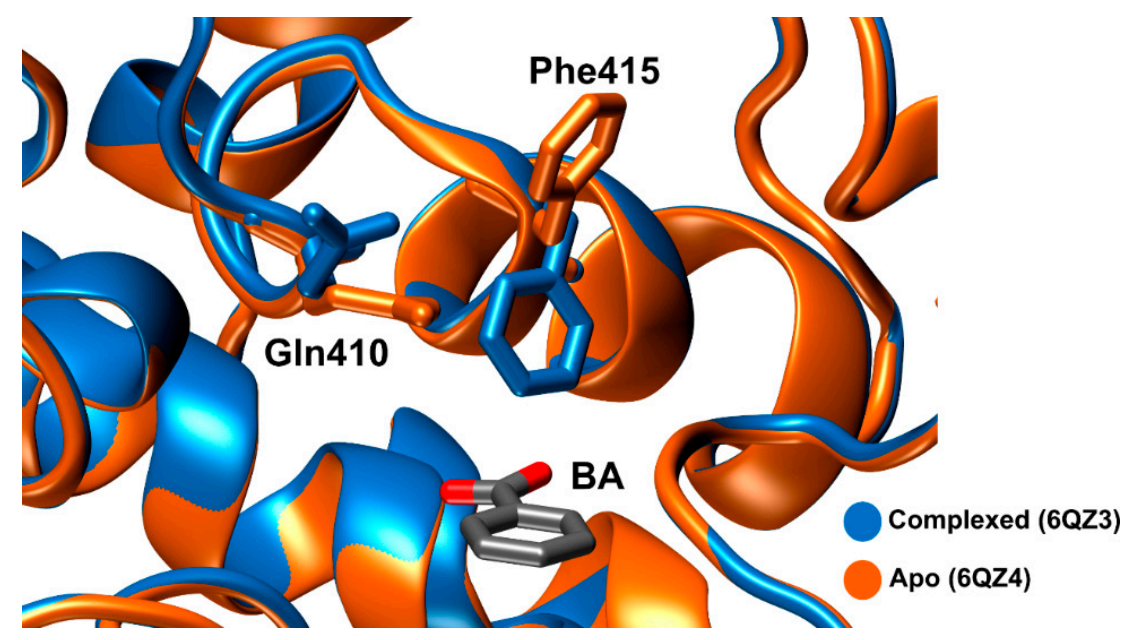

Figure 10. Schematic representation of Phe415 and Gln410 conformational change upon binding to benzoic acid (BA). Apo-form is represented in orange (PDB: 6QZ4) and the complexed form is represented in blue 8PDB: 6QZ3).

\subsubsection{Activity}

IsMHETase has a high affinity and activity towards the substrate MHET, resulting in a $\mathrm{K}_{\mathrm{M}}$ and $\mathrm{k}_{\mathrm{cat}}$ of $7.2 \mu \mathrm{M}$ and $27.6 \pm 2.6 \mathrm{~s}^{-1}$ [75,89]. However, the enzyme is incapable of efficiently degrading the other PET intermediate BHET, resulting in a $\mathrm{k}_{\mathrm{cat}}$ of 
$0.0011 \pm 0.0002 \mathrm{~s}^{-1}$ [80]. The substitution of the catalytic triad by alanine mutants resulted in total loss of enzymatic activity, confirming their essential role in catalysis [80,89]. The oxyanion hole residue Glu226 was mutated to threonine (E226T). The variant resulted in a $\sim 50 \%$ activity reduction [89]. Having five disulfide bonds, IsMHETase was expected to have a relatively high thermostability. However, when analyzing IsMHETase melting temperature, Sagong et al. determined that its $\mathrm{T}_{\mathrm{m}}$ value was only $50.61^{\circ} \mathrm{C}$.

Several engineering efforts to increase IsMHETase performance and stability were conducted. The amino acid residue Trp397, reported to be involved in MHET substrate binding, was replaced by the hydrophobic residue alanine (W397A). The resulting variant manifested an increase of enzymatic activity at high substrate concentration towards MHET and MpNPT (mono-4-nitrophenyl terephthalate) and a slight decrease of substrate affinity of MpNPT [80]. Arg411, reported to establish hydrogen bonds with substrates was replaced by alanine (R411A), positive charged amino acid lysine (R411K) and polar amino acid glutamine (R411Q). As expected, the variants R411A and R411Q have low affinity and activity towards MHET and MpNPT. However, R411K variant has a 1.7-fold activity increase for BHET as a substrate $[80,95]$.

The residue that undergoes induced-fit conformational change, Phe415, has been mutated to alanine, serine, and histidine (F415A, F415S, and F415H, respectively). Interestingly, the variant $\mathrm{F} 415 \mathrm{H}$ exhibits an increased turnover rate towards MHET and has no effect on MpNPT, while F415A resulted in a lower turnover rate and affinity towards MHET and MpNPT and F415S resulted in a lower hydrolysis activity towards MHET [80].

Phe424 is a residue that is located at the inner substrate-binding site and is potentially hindering the optimal BHET binding. Thus, mutagenesis was applied replacing the residue for glutamine, asparagine, histidine, aspartate, glutamate, threonine, valine, leucine, isoleucine, alanine, and serine (F424Q, F424N, F424H, F424D, F424E, F424T, F424V, F424L, F424I, F424A, and F424S, respectively). Overall, all variants resulted in low turnover rates against MpNPT and MHET and, as expected, higher turnover rates and activity towards BHET, where F424N, F424V, F424I, and F424Q display better results. The possibility of IsMHETase having a catalytic tetrad was ruled out when the variant His488 resulted in an unaltered turnover rate [80].

Knott et al. [89] have an interesting approach when it comes to the lid domain and the disulfide bond presented in IsMHETase. The lid domain of IsMHETase (Gly251-Thr472) was removed and replaced by the IsPETase loop residues (Trp185-Phe191), which possibly confer activity against PET. However, the resulting enzyme was unable to degrade PET and the turnover rate value towards MHET was 1000-fold lower when compared to WT IsMHETase. The active site disulfide bond (Cys224-Cys529) was then removed from similar lidless IsMHETase variants, being replaced with tryptophan and serine (C224W-C529S) or histidine and phenylalanine $(\mathrm{C} 224 \mathrm{H}-\mathrm{C} 529 \mathrm{~F})$. These variants conferred almost a total loss of activity against MHET, being the $\mathrm{k}_{\text {cat }}$ values $0.10 \pm 0.06 \mathrm{~s}^{-1}$ and $0.06 \pm 0.03 \mathrm{~s}^{-1}$, respectively. Lastly, the group included disulfide bonds from PETase-like (G489C/S530C) and $A \mathrm{oFaeB}$, giving IsMHETase a total of seven disulfide bonds. The resulting variant had a very low activity towards MHET $\left(\mathrm{k}_{\mathrm{cat}}=0.16 \pm 0.14 \mathrm{~s}^{-1}\right)$. More variants were tested but failed to express.

Besides the described assays, many other engineering efforts have been done on MHETase, as summarized in Table S2.

\subsubsection{Proposed Mechanism}

A proposal for the catalytic mechanism of IsMHETase was made by Knott et al. [89], based on quantum mechanical/molecular mechanical (QM/MM) calculations with 2D umbrella sampling.

The structures determined by the group suggest that the best fitting mechanism for IsMHETase hydrolysis is the one characteristic of the serine hydrolase enzymes. According to this proposal, catalysis involves a two-step reaction, where the formation of an acylenzyme intermediate (acylation) occurs first, and is followed by its hydrolytic release. 
For acylation, His528 is thought to deprotonate Ser225, which becomes a nucleophile and attacks the carbonyl carbon of MHET, resulting in the liberation of EG. This exits the active site within $4 \mathrm{~s}$ of the formation of the acyl-enzyme intermediate (AEI). The minimum free-energy path (MFEP) calculated from the $\mathrm{C}-\mathrm{O}$ bond that was formed between the MHET carbonyl carbon and Ser225 and the broken MHET C-O bond, predicts an acylation free-energy barrier $\left(\Delta \mathrm{G}_{\ddagger}\right)$ of $13.9 \pm 0.17 \mathrm{kcal} / \mathrm{mol}$ and an overall reaction free energy $\left(\Delta \mathrm{G}_{\mathrm{r}}\right)$ of $-5.2 \pm 0.04 \mathrm{kcal} / \mathrm{mol}$. The departure of EG allows a better access for water molecules to interact with charged His528, marking the start of the second step. AEI is subjected to nucleophilic attack by a water molecule, where His528 deprotonates the catalytic water and transfers the proton to the catalytic Ser225, regenerating the former for a new catalytic cycle, thus releasing TPA. The MFEP calculated from the $\mathrm{C}-\mathrm{O}$ bond that was formed between MHET and water and the broken AEI C-O bond predicts a diacylation $\Delta \mathrm{G}_{\ddagger}=19.8 \pm 0.10 \mathrm{kcal} / \mathrm{mol}$ and $\Delta \mathrm{G}_{\mathrm{r}}=2.60 \pm 0.07 \mathrm{kcal} / \mathrm{mol}$. This step is thought to be the rate-limiting step, with a $\mathrm{k}_{\text {cat }}$ of $7.1 \pm 1.1 \times 10^{-2} \mathrm{~s}^{-1}$, while for acylation it is about $1.02 \pm 0.28 \times 10^{3} \mathrm{~s}^{-1}$ [89]. Overall, the reaction is exergonic $(-2.60 \pm 0.08 \mathrm{kcal} / \mathrm{mol})$.

\subsubsection{Future Perspectives}

Many key factors need to be studied for a better understanding of IsMHETase. The enzyme's thermal stability is significantly low when compared with the known necessary values in the industry of plastics, and engineering efforts to solve this issue are essential for further development. Furthermore, confirmation of the catalytic mechanism is needed, which provides needed information for the application of mutagenesis, and lastly, development of new mutant variants is needed to increase the hydrolytic activity of IsMHETase to the plastic-degrading industry standard levels.

\subsection{Pseudomonas Aestusnigri PETase (PaPETase)}

\subsubsection{Discovery}

PaPETase, commonly known as PE-H, is a PET hydrolase produced by the bacterium Pseudomonas aestusnigri, first identified by Bollinger et al. [97] in 2017. P. aestusnigri, a gramnegative rod shaped bacteria, is a novel species isolated from a marine area affected by a large oil spill in the last decade [98]. Motivated by the evidence that these bacteria had polyester degrading activity [99], the bacterial genome sequence [100] revealed a likely hydrolase coding gene [101] that coded for functional PET hydrolase. Wild type PaPETase actively degrades amorphous PET film and BHET to MHET, with no production of TPA.

\subsubsection{Structure}

PaPETase is composed of 304 amino acid residues, with a total molecular weight of $32 \mathrm{kDa}$, plus a signal peptide of 25 amino acids. Bollinger et al. [97] solved two PaPETase structures, summarized in Table 3 . The structure revealed a functional monomer with a conserved $\alpha / \beta$-fold composed of a nine $\beta$-strand central twisted $\beta$-sheet and seven $\alpha$-helices on both sides, as represented in Figure 11.

Table 3. Crystallographic structures of PaPETase.

\begin{tabular}{cccccc}
\hline PDB Code & Resolution (̊̊) & Ligand & Mutation & Year of Deposition & Ref. \\
\hline 6SBN & 1.09 & - & - & 2019 & [97] \\
\hline $6 S C D$ & 1.35 & - & Y250S & 2019 & [97] \\
\hline
\end{tabular}




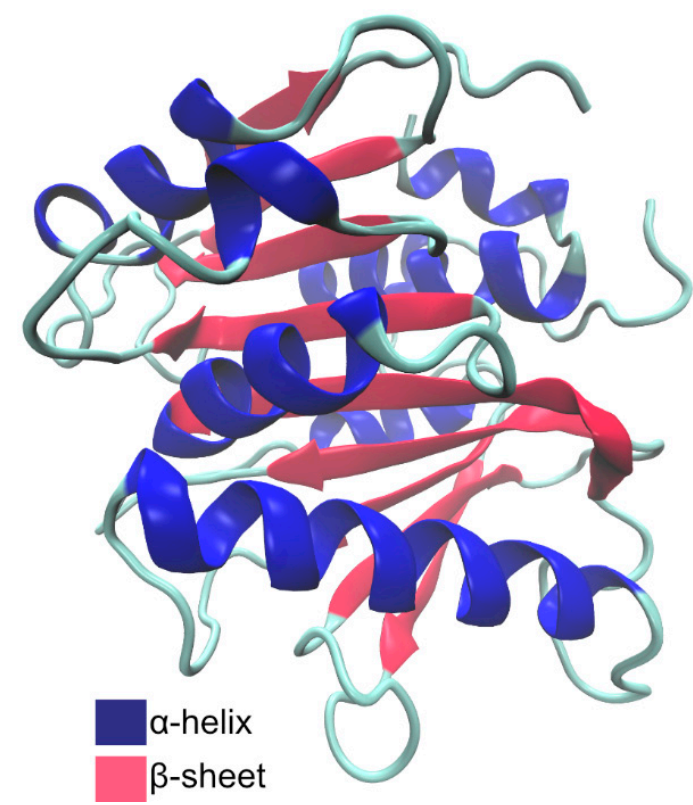

Figure 11. Schematic representation of PaPETase secondary structure motifs.

The catalytic triad (Ser171, Asp217, and His249) is found right below the surface, with Ser171 occupying the traditional position in the nucleophilic elbow. An oxyanion hole composed of Met172 and Phe98 stabilizes the substrate during the reaction. The enzyme has two disulfide bonds-Cys214-Cys251 and Cys285-Cys302, as is common for type II PET hydrolyzing enzymes. Characterization of PaPETase as a Type II PET hydrolase is due to the additional disulfide bond and novel amino acid residues near catalytic histidine residue, not present in Type I PET hydrolyzing enzymes, which are typically cutinases. An extended loop region connecting $\beta 8-\alpha 6$ like the one observed in IsPETase [74] was identified in $P a$ PETase and is defined by residues $254-259$. The amino acid content of this loop defines this enzyme as a type IIa PET hydrolytic enzyme. These relevant structural aspects are represented in Figure 12.

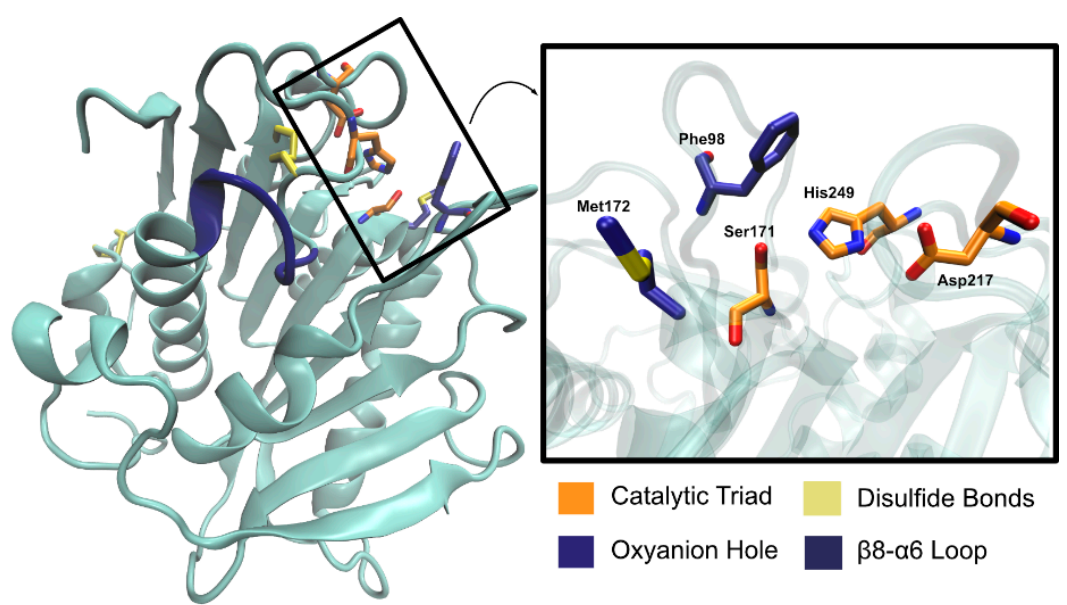

Figure 12. Relevant PaPETase amino acid residues. The catalytic triad, oxyanion hole, and disulfide bond residues are represented in licorice, while the loop is represented in new cartoon.

\subsubsection{Activity}

Bollinger et al. [97] verified that WT PaPETase degraded BHET and amorphous PET to MHET, with almost no TPA production registered. PaPETase degradation of PET resulted in $4.2( \pm 1.6) \mathrm{mg} / \mathrm{L}$ MHET after $40 \mathrm{~h}$ at $30^{\circ} \mathrm{C}$. No hydrolysis on commercial bottle film PET was observed. 
PaPETase and IsPETase are both defined as Type II PET hydrolyzing enzymes. However, $\mathrm{PaPETase}$ is further classified as a type IIa enzyme, while IsPETase belongs in group $\mathrm{IIb}$. This distinction is due to the amino acid content of a structurally conserved extended loop region made up of six amino acid residues. In PaPETase, these are Gly254, Gly255, Ser256, Ile257, Tyr258, and Asn259. On the other hand, the IsPETase loop is made up of Ser242, Gly243, Asn244, Ser245, Asn246, and Gln247. Besides, Tyr250 was identified as the equivalent $\mathrm{PaPETase}$ residue to IsPETase Ser238, a relevant amino acid in IsPETase catalysis. $\mathrm{PaPETase}$ variants containing single-point and entire loop replacements in these positions with IsPETase residues were produced and tested for activity against BHET and PET. Variants G254S, Y258N, N259Q, and the full loop mutation resulted in significantly decreased activity and lower $\mathrm{T}_{\mathrm{m}}$ than WT enzyme by $5-10{ }^{\circ} \mathrm{C}$. PaPETase S256N, I257S, and also resulted in diminished activity but less drastic lowering of $\mathrm{T}_{\mathrm{m}}$, by $1-3{ }^{\circ} \mathrm{C}$. Only Y250S resulted in higher activity against $p$ NPB, BHET, and PET, resulting in a higher production of MHET than wild type enzyme. Particularly, variant Y250S yielded $5.4( \pm 0.6) \mathrm{mg} / \mathrm{L}$ of MHET after $48 \mathrm{~h}$ at $30^{\circ} \mathrm{C}$, higher than the $4.2 \mathrm{mg} / \mathrm{L}$ produced by WT enzyme. Variants Y250S and S256N were active on film PET derived from a commercial bottle, unlike WT $\mathrm{PaPETase}$ enzyme, although with a relative low yield.

Structure determination of variant Y250S (PDB: 6SCD) revealed a more accessible and spacious active site cleft when compared with WT PaPETase (PDB: 6SBN). Two loop regions (the loop connecting $\beta 3-\alpha 2$, composed of residues 98-104, and loop connecting $\beta 4-\alpha 3$, made up of residues 123-128) are mostly responsible for these differences. In the WT enzyme, these loops are parallel to each other in a "closed" conformation, while in the engineered variant they are shifted against each other, which allows for more space in the catalytic site. The loops responsible for the tightening of the active site in the WT enzyme are stabilized by the interaction between Tyr250 and Glu102. Through the disruption of this interaction, variant Y250S induces structural rearrangements, which enlarge the volume of the active site cavity from $153 \AA^{3}$ to $362 \AA^{3}$, resulting in a deeper and more substrate accessible cleft, which contributes to increased hydrolytic activity. All mutations explored in this section are summarized in detail in Table S3.

The enzyme-binding mode was predicted through molecular docking studies with PET tetramer 2-HE(MHET $)_{4}$, MHET, and BHET on both structures. BHET and MHET were predicted to bind in a groove adjacent to the catalytic site, stabilized by interactions with Ser256, Ser248, Asp106, and Ser104. This binding position is not optimal for catalysis, suggesting a necessary conformational change in the active site for interaction between catalytic serine residue and substrate molecules. Furthermore, no valid binding mode for 2-HE(MHET $)_{4}$ was found, due to the narrowness of the active site cleft. Molecular docking with engineered variant Y250S revealed a more favorable binding mode for MHET and BHET, at an appropriate distance for Ser171 attack. Yet again, no valid binding mode for 2-HE(MHET) $)_{4}$ was obtained.

\subsubsection{Proposed Mechanism}

Inspired by their structural and computational studies, Bollinger et al. [97] proposed a mechanism for $\mathrm{PaPETase}$ hydrolysis of PET. The proposal suggests a three-moiety substratebinding mode- one unit would bind the catalytic site adjacent groove, and the other two would bridge the distance to the catalytic site, where the third unit would bind at optimal distance for serine mediated cleavage. The substrate-length dependent catalytic mechanism is similar to the nick and digestion mechanism suggested for IsPETase.

\subsubsection{Future Perspectives}

The similarity to IsPETase and increased activity of $\mathrm{PaPETaseY} 250 \mathrm{~S}$ variant is a strong argument in favor of the proposed binding mode, yet further validation through experimental and computational studies is required. Furthermore, given $\mathrm{PaPETase}$ similarity to IsPETase, reproducing the successful mutations tested on this enzyme could be a promising strategy for enhancing PaPETase activity, complemented with computational strategies 
such as molecular dynamics simulations. However, this mechanistic proposal should be strengthened by experimental evidence on the binding mode (e.g., three-dimensional structures of complexed PaPETase), and computational studies on the active site conformational flexibility through molecular dynamics, in addition to quantum mechanics studies using, for example, a hybrid QM/MM methodologic approach [102-104].

\subsection{LC-Cutinase (LCC)}

\subsubsection{Discovery}

LCC, a cutinase homologue, was first isolated in 2011 from a leaf-branch compost through a metagenomic approach [58]. Even though the source organism for this enzyme remains to be identified, it is presumed to be thermophilic bacteria. This assumption results from the high sequence identity between LCC and bacterial cutinases (in comparison to fungal cutinases), and the temperature of the compost from which LCC was firstly isolated $\left(67^{\circ} \mathrm{C}\right)$ [105]. LCC is a secretory protein with high sequence identity to lipases and cutinases. Specifically, the highest amino acid sequence identity identified is to Thermobifida fusca $(57.4 \%)$ [58]. The enzyme was found to have hydrolytic activity against various fatty acid monoesters and is efficient in degrading PET and depolymerizing poly( $\varepsilon$-caprolactone) (PCL) $[58,106]$.

\subsubsection{Structure}

LCC is composed of 258 amino acid residues (amounting to a molecular mass of $28 \mathrm{kDa}$ ), plus a 34-residue signal peptide at the N-terminus [58,105]. The overall LCC structural fold belongs to the $\alpha / \beta$-hydrolase superfamily and is made up of nine-stranded $\beta$-sheet and eight $\alpha$-helices [105]. The enzyme was determined to be a monomer in the absence of substrates [105]. The surface of LCC has been described as highly charged [107].

In the active site, three residues were found to form a catalytic triad (Ser165, Asp210, His242) and two residues constitute an oxyanion hole (Met166 and Tyr95) [58], as represented in Figure 13. The catalytic serine residue is found in a nucleophilic elbow, a sharp turn between strand $\beta 5$ and helix $\alpha 5$ [105], within a typical GxSxG (GHSMG in LCC) motif $[58,105]$. Comparisons with $T a C u t 1$, whose binding mode is known, resulted in the prediction that a hydrophobic patch made up of residues Tyr95, Thr96, Ala97, Phe125, Tyr127, Met166, Trp190, Thr211, Val212, and Phe243 constitutes a protruding long groove from the catalytic pocket, accommodating the binding of long-chain substrates [105]. Even so, a specific binding mode remains to be fully understood.

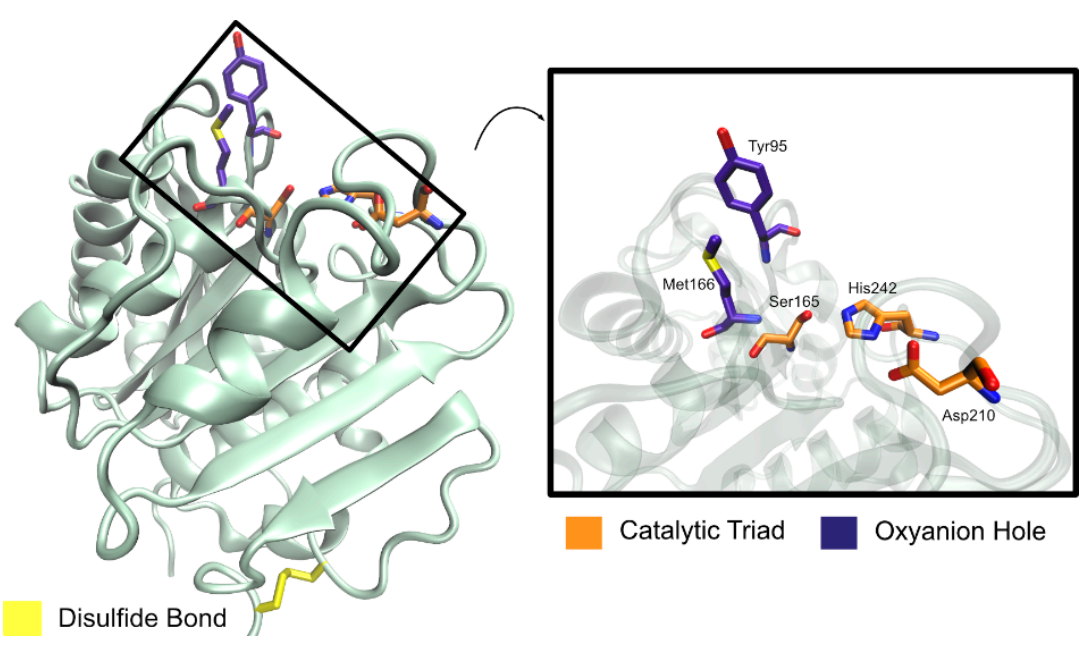

Figure 13. Schematic representation of LCC catalytic triad, oxyanion hole, and disulfide bond amino acid residues, and relative position in overall protein structure.

A disulfide bond (Cys275-Cys292) is found to anchor the LCC C-terminus to a strand $\beta 9-$ helix $\alpha 8$ loop, similar to other PET hydrolytic enzymes. This bridge is typically respon- 
sible for a higher thermal stability of the enzymes since it exists in thermophilic bacterial cutinases, but not in fungal cutinases [105].

The currently known three-dimensional structures of LCC are summarized on Table 4. All structures exist in apo-form, so no crystallographic data of complexed enzymes are presently available. The first structure was solved at the time of enzyme identification with a resolution of $1.5 \AA$ in 2012 by Sulaiman et al. [58], and allowed authors to confirm the structured features predicted from its sequence and similarity to other enzymes. The remaining available structures, deposited in 2019 by Tournier et al. [106], are engineered versions of LCC.

Table 4. Crystallographic structures of LCC.

\begin{tabular}{cccccc}
\hline PDB Code & Resolution (̊) & Ligand & Mutations & Year of Deposition & Ref. \\
\hline 4EB0 & 1.5 & Free & - & 2012 & {$[105]$} \\
\hline 6THS & 1.10 & Free & S165A & 2019 & {$[106]$} \\
\hline \multirow{2}{*}{ 6THT } & \multirow{2}{*}{1.14} & Free & $\begin{array}{c}\text { Y127G/S165A/ } \\
\text { D238C/F243I/S283C }\end{array}$ & 2019 & {$[106]$} \\
\hline
\end{tabular}

\subsubsection{Activity}

LCC activity was first measured against $p$ NP-butyrate by Sulaiman et al. [58], to determine optimal hydrological $\mathrm{pH}$ and temperature. The enzyme exhibited highest activity at $\mathrm{pH} 8.0$ (with $\sim 70 \%$ of maximal activity at $\mathrm{pH} 7.0$ and 9.5) and temperature of $50{ }^{\circ} \mathrm{C}$ (with $70 \%$ of maximal activity at 30 and $70{ }^{\circ} \mathrm{C}$ ). Measured activity was not changed in the presence of $\mathrm{CaCl}_{2}$ or EDTA. To analyze substrate specificity, several $p$ NP monoesters of fatty acids with different lengths acyl chains of 2 to 12 were used as substrates. The enzyme showed preference towards $p$ NP-butyrate (C4) and almost equal efficiency with $p$ NP-caprylate (C8) and $p$ NP-hexanoate (C6). Acyl chain lengths above 12 resulted in much lower rates-enzymatic activity decreases as chain lengths of substrate increases. The enzyme was found to degrade cutin at a similar rate to T. fusca.

LCC ability to degrade PCL and PET was also tested by Sulaiman et al. [58]. Specific enzymatic activity against PCL was determined to be $300 \mathrm{mg} / \mathrm{h} / \mathrm{mg}$ enzyme at $\mathrm{pH} 8.0$ and $50{ }^{\circ} \mathrm{C}$. Regarding PET degradability, the enzyme degraded $1.45 \mathrm{mg}$ of PET film after $24 \mathrm{~h}$ incubation, resulting in a specific activity of $12 \mathrm{mg} / \mathrm{h} / \mathrm{mg}$ enzyme at $\mathrm{pH} 8.0$ and $50{ }^{\circ} \mathrm{C}$. TPA was identified as the major degradation product, with residual MHET also registered. No BHET monomers were detected. This means that LCC completely hydrolyses PET to TPA and EG. This study reported LCC activity to be 230-970-fold higher than that of other cutinases with PET hydrolyzing activity.

As mentioned, LCC showed highest activity at $50^{\circ} \mathrm{C}$. However, the enzyme remains stable up to $75^{\circ} \mathrm{C}$. Sulaiman et al. [105] concluded that activity decreases as temperature increases above $50{ }^{\circ} \mathrm{C}$, even before enzymatic denaturation. To further investigate this, binding affinity constants $\left(\mathrm{K}_{\mathrm{M}}\right)$ and turnover numbers $\left(\mathrm{k}_{\mathrm{cat}}\right)$ were determined at 30, 50, and $70{ }^{\circ} \mathrm{C} . \mathrm{K}_{\mathrm{M}}$ values were similar, independently of the temperature $(0.21-0.24 \mathrm{mM})$, while $\mathrm{k}_{\text {cat }}$ numbers differ with the change in temperature. This means that a lowering in activity at 60 and $70{ }^{\circ} \mathrm{C}$ is not due to a decrease in binding affinity but to a decrease in the turnover number. Using $p$ NP-butyrate as substrate, $K_{M}$ and $k_{\text {cat }}$ of LCC are $0.21 \mathrm{mM}$ and $343 \mathrm{~s}^{-1}$ at $50{ }^{\circ} \mathrm{C}$, considerably higher than known values for other cutinases. Unlike $p \mathrm{NP}$-butyrate, PET is a long-chain substrate that interacts with various binding site residues, differing in this regard from short chain monomers. Activity assays against PET film showed that activity increased at $70{ }^{\circ} \mathrm{C}$ - optimal enzymatic activity is higher at higher temperatures. This effect is likely due to changes in crystallinity PET suffers at higher temperatures and stabilization of the active by the long-chain substrate.

The role of catalytic serine residue Ser165 was confirmed by mutagenesis to alanine (S165A), leading to an almost total loss of activity [58,106]. 
A double mutant disrupting the disulfide bridge (C275A/C292A) was produced by Sulaiman et al. [105] to investigate the predicted stabilizing role of this structural attribute. Spectroscopy studies showed the overall fold of LCC was not affected by this mutation. Measurement of denaturation curves showed a destabilization of approximately $15^{\circ} \mathrm{C}$ when compared to WT enzyme, enforcing the essential role of the disulfide bridge in thermal stability. $\mathrm{LCC}(\mathrm{C} 275 \mathrm{~A} / \mathrm{C} 292 \mathrm{~A})$ measured activity at $30^{\circ} \mathrm{C}$ was comparable to that of WT LCC at $50{ }^{\circ} \mathrm{C}$, indicating that active site stability is affected by the overall enzymatic fold stability.

Tournier et al. [106] compared LCC activity against commercially available amorphous PET with several know PET hydrolyzing enzymes (TfHCut and BTA-2, FsCut and IsPETase). LCC outperformed all tested enzymes in PET degradation, proven to be at least 33 times more efficient with an optimal activity temperature of $65^{\circ} \mathrm{C}$. Furthermore, high thermal stability was confirmed by determination of $\mathrm{T}_{\mathrm{m}}$ at $84.7^{\circ} \mathrm{C}$. Even so, once again activity decreased as temperature increased, despite high thermal stability, motivating the authors to attempt to increase enzymatic stability and activity simultaneously. Molecular docking and molecular dynamics calculations were performed to predict binding mode of a 2-HE(MHET) ${ }_{3}$ chain. Predicted binding site was consistent with the conserved protruding hydrophobic groove previously described, and inspired 11 site-specific mutations, with production of 209 novel variants. Of these variants, F243I and F243W resulted in improved activity, while T96M, Y127G, N246D, and N246M showed similar activity but higher melting temperature values. In an attempt to further increase thermal activity, an additional disulfide bond (D238C/S283C) was introduced, leading to a $\mathrm{T}_{\mathrm{m}}$ of $94.5^{\circ} \mathrm{C}$ (increased by $9.8^{\circ} \mathrm{C}$ ) with only $28 \%$ loss of activity. Two variants combining the disulfide bridge and the activity increasing mutations were engineered: ICC (F243I/D238C/S283C) and WCC (F243W/D238C/S283C). ICC and WCC presented similar activity to WT enzyme but higher melting temperature values. Finally, eight new variants combining ICC and WCC with previously described T96M, Y127G, N246D, and N246M single-point mutations were generated. Of these, four variants (ICCG, ICCM, WCCG, and WCCM) resulted in similar or higher activity than WT-LCC and improved $\mathrm{T}_{\mathrm{m}}$ up to $13.4^{\circ} \mathrm{C}$. Exact measurements for these variants and all known LCC mutated enzymes are summarized in Table S4. A three-dimensional structure of variant ICCG was solved at $1.14 \AA$ (PDB: 6THT). Molecular dynamics and MM/GBSA calculations with this structure and 2-HE(MHET) 3 showed increased affinity and facilitated productive catalytic binding of substrate, further confirming the efficiency of this variant.

Shirke et al. [107] attempted glycosylation to avoid LCC aggregation, under the rationale that reduced aggregation would result in higher kinetic stability. LCC presented aggregation tendencies in its native state resulting from ionic interactions. Glycosylation (covalent binding of an oligosaccharide to protein) may stabilize enzymatic conformation and impose steric constraints inhibiting protein-protein interactions, reducing aggregation. Glycosylated LCC (LCC-G) showed higher thermal stability and increased activity against PET at higher temperatures when compared with non-glycosylated protein. Furthermore, unlike what had been observed for WT enzyme, with LCC-G activity did not decrease with increasing enzymatic concentration, but remained constant once a given activity maximum was attained. LCC-G $\mathrm{T}_{\mathrm{m}}$ is reportedly $12{ }^{\circ} \mathrm{C}$ higher than non-glycosylated LCC.

Yan et al. [108] developed a thermophilic whole-cell biocatalyst with high LCC expression to efficiently degrade PET film. Whole-cell biocatalysis allows for simultaneous enzyme production and hydrolysis in a single step-meaning, the system can produce functional LCC and degrade substrates simultaneously with consistent reaction conditions. Clostridium thermocellum (thermophilic anaerobe bacterium with an optimal growth temperature of $60^{\circ} \mathrm{C}$ ) was used to generate a whole-cell biocatalyst with high secretory expression of LCC at high temperatures. PET hydrolytic activity was registered. Over $60 \%$ weight loss of an amorphous PET sample was observed over a 14-day incubation period, resulting in a degradation rate higher than $2.2 \mathrm{mg} /$ day, which is higher than previous whole-cell PET hydrolyzing systems reported. This strategy is frequently simpler, less expensive, 
and more efficient than biodegradation with purified free enzymes, and this study is highly promising for the future of biocatalysts in plastic biodegradation.

\subsubsection{Future Perspectives}

Although LCC was first isolated in 2012, several questions remain to be answered. The lack of a protein-ligand three-dimensional structure and accurate binding mode characterization hinders the suggestion of a solid catalytic mechanism. Even so, in recent times this enzyme has received high attention from the scientific community, given the evidence of its high-level activity and thermal stability when compared with other PET hydrolases. The high performance of the few engineered versions developed is promising, and it is to be expected that further mutagenic and activity studies will be successful in enhancing enzymatic activity. Furthermore, since LCC is stable at much higher temperatures than, for example, IsPETase, potential for activity above PET's glass temperature is highly attractive.

2.5. Thermomonospora fusca Hydrolase (TfHCut) and Thermomonospora fusca BTA Hydrolase 2 (BTA-2)

\subsubsection{Discovery}

TfHCut, Thermomonospora fusca hydrolase, also referred to as TfH, BTA-hydrolase 1 (BTA-1) [109] or Tfu_0883 [110] is a type I PET degrading cutinase-like enzyme. The ability of T. fusca, a thermophilic filamentous soil bacterium [111], to degrade polyester-like substrates, was first identified by Kleeberg et al. [109] in 1998 with BTA, a copolyester of 1,4-butanediol, TPA, and adipic acid.

The initial ability to degrade BTA earned TfHCut the alternative name of BTA-1 [112]. Another enzyme expressed by T. fusca also showed BTA and PET degradation activity and was therefore termed BTA Hydrolase-2 (BTA-2). Even though this enzyme has received considerably less attention than BTA-1, they share $92 \%$ amino acid identity and the genes that express these proteins are nearly identical [112].

$\mathrm{T} f \mathrm{HCut}$ was later identified, purified, expressed, and characterized as a thermophilic hydrolase with ability to degrade aliphatic-aromatic copolyesters [113]. The different names this enzyme is known as are due to the uncertainty in classifying it as a lipase or cutinase. When Chen et al. [110] identified specific cutinase-like activity, they renamed $\mathrm{T} f \mathrm{HCut}$ as $\mathrm{Tfu}$-0883, and to this day both designations, as well as BTA-1, are used.

Specific PET degrading activity was first shown by Müller et al. [51] with two different PET samples-commercial bottle PET and PET-B in pellets. TfHCut ability to hydrolase PET was further analyzed and enhanced by Silva et al. [55] and Then et al. [114].

\subsubsection{Structure}

Tf HCut is composed of 261 amino acids with a total molecular weight of $28 \mathrm{kDa}$ [113]. It is, similar to most PET hydrolases, a serine hydrolase with Ser170, His248 and Asp216 as the catalytic triad [110]. Catalytic Ser is found in a G-H-S-M-G conserved sequence [113]. in a nucleophilic elbow [110]. A homology model built by Chen et al. [110] based on SeLip, a S. exfoliates lipase, revealed an oxyanion hole formed by Met171 and Tyr100, and confirmed the $\alpha / \beta$-hydrolase general fold up made up of a central $\beta$-sheet with $\alpha$ helices on both sides. These findings were later confirmed by Silva et al. [55] that also produced a homology model with a similar protocol.

Kleeber et al. [113] predicted an exposed and not buried binding site, facilitating the attack to the cleavable ester bonds, which Silva et al. [55] later confirmed.

The only three-dimensional structure of $T f \mathrm{HCut}$ available was recently released by Dong et al. [115] (PDB: 5ZOA). This structure resolved at $1.54 \AA$ contains all the 261 amino acids, suggests the enzyme is a functional monomer, and confirmed the catalytic triad and oxyanion hole residues predicted by homology years earlier. The authors were unable to obtain structures crystalized with BHET and several cutin mimics. Therefore, molecular docking and molecular dynamics calculations were employed to study the active site and 
binding mode. Molecular Docking with an oligo-polyester $\left(\mathrm{C}_{24} \mathrm{H}_{42} \mathrm{O}_{8}\right.$, CAS number 1006130-0) that mimics cutin revealed that part of the substrate was inserted in the hydrophobic shallow groove and part was exposed to the bulk solvent environment. An ester bond was located near the catalytic triad, in an optimal binding mode for catalysis to occur. Furthermore, the docked ligand formed hydrogen bonds with the oxyanion hole residues. Given the bulky residues located at either side of the binding groove, it was theorized that availability for substrate accommodation was not optimal. Therefore, these and other structural findings revealed by the structure were used as rationale for protein engineering and activity assays, described below.

Visual inspection of the available PDB structure and comparison with similar PET degrading cutinases suggests a disulfide bridge between residues Cys299 and Cys281.

\subsubsection{Activity}

Kleeberg et al. [113] showed that the ability of TfHCut to degrade BTA was enhanced by the addition of pectin, peptone, and tryptic soy broth to the medium, and was unaffected by the addition of polysaccharides. Maximum growth temperature for T. fusca was determined at $55^{\circ} \mathrm{C}$, and maximum $\mathrm{TfHCut}$ activity was registered at $65^{\circ} \mathrm{C}$ and $\mathrm{pH} 6.0$ to 6.5 , which is a typical behavior for extracellular enzymes. Activity rapidly decreased at $70{ }^{\circ} \mathrm{C}$, likely due to enzymatic denaturation. Ester bond cleavage ability of $\mathrm{T} f \mathrm{HCut}$ was demonstrated with several triglycerides of varying chain lengths. This activity was higher for shorter chain polymers, decreasing as length increased. Hydrolytic activity on aliphatic polyesters (PCL, commercial biodegradable plastic Bionelle, and SP3/13 and Bayer Tir 1874) was also demonstrated, but no degradation of PHB was attained. Activity assays and predicted characteristics derived from sequence determination led to the definition of $\mathrm{T} f \mathrm{HCut}$ as a lipase, since specific cutinase-like activity was not confirmed.

PET hydrolytic activity was analyzed by Müller et al. [51] using two different PET samples-commercial bottle PET and a PET-B sample obtained as a pellet. Rates of depolymerization were similar for both samples over a three-week incubation period. The erosion rate was 8-17 $\mu \mathrm{m}$ week ${ }^{-1}$ per film side. Degradation of PET was performed at $55^{\circ} \mathrm{C}$, and DSC experiments confirmed low crystallinity of the PET samples used at this temperature, facilitating enzyme binding and activity rates. Assays with PHB and PBT were also conducted, with no sample weight loss registered.

Silva et al. [55] engineered two new variants of $T f H C u t$ with different active site residues, as a strategy to promote active site enlargement (I218A) and to increase hydrophobicity (Q132A/T101A). PET degrading activity was measured with PET fabric at $60{ }^{\circ} \mathrm{C}$ and activity rate was determined by measuring amounts of TPA release, since the enzyme can fully degrade PET to TPA. The engineered variants degraded PET to TPA with a two-fold activity increase when compared with the WT enzyme. The mutants achieved a higher catalytic efficiency and higher levels of protein adsorption than WT TfHCut, probably due to the increased active site binding space and enforced hydrophobic character. Further catalytic assays revealed Q132A/T101A to be the better-performed mutant, with a much higher catalytic rate than WT enzyme.

Chen et al. [110] determined esterase, cutinase, and lipase activity of $\mathrm{TfHCut}$. Esterase activity was measure with $p \mathrm{NPB}$ as a substrate at $\mathrm{pH}$ 8.0. Cutinase activity was determined in similar conditions using apple cutin as a substrate, and for lipase activity measurement, triolein was selected as substrate. Enzymatic thermal stability was explored by repeating $p$ NPB activity assays at temperatures ranging from 20 to $60^{\circ} \mathrm{C}$. Through verification of high cutinase-like activity, TfHCut, at the time known mostly as TfH or BTA-1, was renamed Tfu_0883 and considered a cutinase.

Then et al. [114], inspired by the effect $\mathrm{Ca}^{2+}$ had on LCC and TfCut1 activity, explored the activity of Tf HCut and BTA-2 in the presence of $\mathrm{Ca}^{2+}$ and $\mathrm{Mg}^{2+}$ ions. The PET degrading assays were performed on PET film substrate at $\mathrm{pH} 8.5$ and $50{ }^{\circ} \mathrm{C}$ for $48 \mathrm{~h}$ on a shaker at $125 \mathrm{rpm}$. The effect on the cations on PET hydrolase activity was dependent on the reaction temperature. At $55{ }^{\circ} \mathrm{C}$, the activity was similar to the WT enzyme. On the other 
hand, activity at $65^{\circ} \mathrm{C}$ was only possible in the presence of $\mathrm{Ca}^{2+}$ or $\mathrm{Mg}^{2+}$ ions. TfHCut had a 4.5-fold activity increase at $65^{\circ} \mathrm{C}$ in the presence of the ions when compared to the WT enzyme performance at $60^{\circ} \mathrm{C}$. Molecular dynamics simulations to determine probable ion binding sites were performed, and concluded that $\mathrm{Ca}^{2+}$ was likely to bind Asp174 and Asp204, via the carboxyl groups, and to Gly205 via the amide hydrogen. $\mathrm{Mg}^{2+}$ was suggested to bind to five residues-Glu253, Asp174, Asp246, Glu26, and Thr41. Given the positive effects that these cations have on the activity and thermal stability, and the recently known structure of $\mathrm{Ca}^{2+}$ bound $\mathrm{T} f \mathrm{HCut}$, further computational studies through molecular dynamics, free energy, and QM/MM calculations could be performed to understand PET-degradation activity.

Recently, Dong et al. [115], inspired by the TfHCut structure solved (PDB: 5ZOA), performed several mutations to increase activity on $p$ NPB and tomato cutin. Two sets of bulky residues were mutated to alanine residues. The first set included residues near the catalytic triad that might hinder availability of substrate to the catalytic residues. The second set (contained residues near the substrate-binding site in order to increase accessibility for longer chain substrates. In the assays with $p$ NPB, Y100A, T247A, and F249A from the first set and L130A and I253A from the second set resulted in enhanced activity. Regarding the tomato cutin experiments, only L130A and I253A (and the combined mutation L130A/I253A) resulted in activity increase. Therefore, molecular docking experiments with the L130A/I253A variant were performed, revealing that the mutation resulted in better substrate accommodation and increased binding site flexibility. These mutations and their effects on enzymatic performance are summarized in Table S5.

Alisch-Mark et al. [116] showed TfHCut ability to modify PET fibers surface, something essential for biodegradation in the textile industry. The role of the catalytic triad was demonstrated by Chen et al. [110]; the effect of PMFS (mechanism-based serine hydrolase inhibitor) on enzymatic activity was measured, with almost no activity registered. Furthermore, the mutation of catalytic serine to alanine also resulted in total loss of activity, confirming the predicted catalytic activity.

TfHCut is an extracellular acting enzyme, as demonstrated by Su et al. [117], by showing hydrolytic activity of phospholipids. The over-expression of the enzyme in various organisms, to achieve optimal quantity and purity levels, has been achieved in several studies $[112,118,119]$, which is a promising experimental milestone for further enzymatic engineering studies.

\subsubsection{Proposed Mechanism}

Chen et al. [110], inspired by the predicted homology model, suggested a classical $\alpha / \beta$-hydrolase catalytic mechanism performed by the catalytic triad (Ser170, His248, Asp216) stabilized by an oxyanion hole (Met171 and Tyr100) and involving formation of two tetrahedral transition states and an acyl-enzyme intermediate.

\subsubsection{Future Perspectives}

TfHCut was the first PET hydrolase identified and characterized, and has inspired countless organism and enzyme identification campaigns, leading to the discovery of many PET degrading cutinases. The many designations that the enzyme has acquired through the years hinder a good overview of activity and performance. On the other hand, the multiple available studies mean there is more available information on this enzyme than for many other potential PETase-like enzymes. The recent deposition of a three-dimensional structure coupled with experimental evidence of $T f \mathrm{HCut}$ ability to fully degrade PET to TPA are promising factors. A deeper understanding of the PET or PET-like substrate binding mode to TfHCut through molecular docking [120-124] and molecular dynamics simulations [125-130] could be used not only to understand the molecular rationale behind the performance of the many variants developed, but also to rationally inspire the design of further mutations. 


\subsection{Saccharomonospora viridis AHK190 Cutinase (SvCut190)}

\subsubsection{Discovery}

Saccharomonospora viridis AHK190 Cutinase (SvCut190, also referred to as Cut190) is a PET degrading-enzyme from Saccharomonospora viridis AHK190, first identified for its PETase-like activity by Kawai et al. [56] in 2014. S. viridis is a thermophilic bacterium with an optimal temperature for growth of $55^{\circ} \mathrm{C}$ [131]. The enzyme contains 304 amino acids residues and is only active in the presence of $\mathrm{Ca}^{2+}$ ions.

\subsubsection{Structure}

SvCut190 exists as a monomer and adopts an $\alpha / \beta$-hydrolase fold, with a central twisted $\beta$-sheet of nine $\beta$-strands and six $\alpha$-helices. No lid domain was identified covering the active site. Short helices $\alpha 1, \alpha 4, \eta 1$, and $\eta 2$ have also been identified. A conserved pentapeptide sequence motif and a typical catalytic triad of the lipase family, composed of Ser176, His254, and Asp222, which are located on the loops $\beta 5-\alpha 5, \beta 7-\alpha 6$, and $\beta 8-\alpha 7$, respectively, have been observed [132]. Residues Phe106 and Met177 have been shown to define an oxyanion hole. A disulfide bond (Cys287-Cys302) that connects the terminal loop to the $\alpha 8-\beta 9$ loop and three $\mathrm{Ca}^{2+}$ binding sites, which are located on the $\beta 1-\beta 2, \beta 7-$ $\beta 9$ and $\beta 6-\beta 7$ loops, respectively [133] are also present. After binding to $\mathrm{Ca}^{2+}$, the enzyme undergoes large conformational changes, which induces the pocket to open providing an easier access for substrate binding [133]. Oda et al. [134] identified the residues involved in the three $\mathrm{Ca}^{2+}$ binding sites. The $\mathrm{Ca}^{2+}$ binding site located in the $\beta 1-\beta 2$ loop (Site 1) has an important role on enzymatic activity, and is made up of Ser76, Ala78, and Phe81. Site 2 is in the $\beta 7-\beta 9$ loop, contributes to thermostability, and involves the amino acids Glu220, Asp250, and Glu296. Site 3, located in the $\beta 6-\beta 7$ loop, contains amino acid residues Asp204 and Thr206, and is related to both activity and thermostability.

The first crystallographic structures were determined in apo-form by Miyakawa et al. [132] in 2014, complexed with $\mathrm{Ca}^{2+}$ (1.75 and 2.35 A resolution, PDB: 4WFJ and 4WFK, respectively) and without $\mathrm{Ca}^{2+}(1.45 \AA$ resolution, PDB: 4WFI). The three structures have a mutation on the position 226, where Ser226 was replaced with proline (S226P). In a later report, the same group determined the crystal structures of $S v$ Cut190S176A/S226P/R228S bound to $\mathrm{Ca}^{2+}$ and $\mathrm{Zn}^{2+}$ (1.60 and 1.22 $\AA$ resolution, PDB: 5ZNO and 5ZRQ, respectively) and complexed with Et-succinate and Et-adipate (1.34 and 1.40 ̊̊ resolution, PDB: 5ZRR and 5ZRS, respectively) [133]. When the mutated enzyme is complexed with Et-succinate, conformational changes on $\beta 3-\alpha 2$ and $\beta 4-\alpha 3$ loops (designated as the engaged form) are observed. Trp107, which forms a hydrogen bond with Arg135 in the ligand free form, undergoes induced-fit conformational change and interacts with the ligand ester group.

Other SvCut190 crystal structures available in the Protein Data Bank are summarized and characterized in Table 5.

Table 5. Crystallographic structures of SvCut190.

\begin{tabular}{|c|c|c|c|c|c|}
\hline PDB Code & Resolution (̊̊) & Ligand & Mutation & Year of Deposition & Ref. \\
\hline $4 \mathrm{WFK}$ & 2.35 & Free & S226 $\mathrm{Pa}^{2+}$ bound & 2014 & [132] \\
\hline $4 \mathrm{WFI}$ & 1.45 & Free & $\mathrm{S} 226 \mathrm{P} \mathrm{Ca}^{2+}$ free & 2014 & [132] \\
\hline $4 \mathrm{WFJ}$ & 1.75 & Free & S226 $\mathrm{PCa}^{2+}$ bound & 2014 & [132] \\
\hline $5 \mathrm{ZNO}$ & 1.6 & Free & S176A/S226P/R228S & 2018 & [133] \\
\hline $5 Z R Q$ & 1.12 & Free & S176A/S226P/R228S & 2018 & [133] \\
\hline 5ZRR & 1.34 & Monoethyl succinate & S176A/S226P/R228S & 2018 & [133] \\
\hline 5ZRS & 1.4 & Monoethyl adipate & S176A/S226P/R228S & 2018 & [133] \\
\hline
\end{tabular}


Table 5. Cont.

\begin{tabular}{cccccc}
\hline PDB Code & Resolution $(\AA)$ & Ligand & Mutation & Year of Deposition & Ref. \\
\hline 7 CEH & 1.09 & Free & $\begin{array}{c}\text { S176A/S226P/R228S with } \\
\text { C-terminal three residues deletion }\end{array}$ & 2020 & $\begin{array}{c}\text { S226P/R228S with C-terminal } \\
\text { three residues deletion }\end{array}$ \\
\hline 7 CEF & 1.6 & Free & $\begin{array}{c}\text { S226P/R228S/Q138A/ } \\
\text { D250C-E296C/Q123H/N202H }\end{array}$ & 2020 \\
\hline 7 CTR & 1.20 & Free & S176A/S226P/R228S/Q138A/ & 2020 \\
\hline 7CTS & 1.10 & Free & D250C-E296C/Q123H/N202H & {$[136]$} \\
\hline
\end{tabular}

\subsubsection{Activity}

The role of Ser176 as a catalytic triad amino acid residue was confirmed when the its mutation to alanine (S176A) totally abolished the enzymatic activity [133]. Kawai et al. [56] determined the $\mathrm{T}_{\mathrm{m}}$ of WT SvCut190 at $55.4{ }^{\circ} \mathrm{C}$.

To date, several engineering efforts have been performed on $S v C u t 190$. As summarized in Table S6, TaEst119 has a conserved proline in position 219 (corresponding to 226 in SvCut190), and due to the similarities of SvCut190 to TaEst119, the variant S226P was developed, resulting in an enhancement of $\mathrm{T}_{\mathrm{m}}$ value by $3.7^{\circ} \mathrm{C}\left(59.1^{\circ} \mathrm{C}\right)$ and enzymatic activity towards $p$-nitrophenyl butyrate. The positively charged Arg228 can potentially form a salt bridge with a negatively charged residue and influence the reaction. Therefore, Kawai et al. [56] replaced Arg228 with neutral serine, producing the variant S226P/R228S in the presence of $\mathrm{Ca}^{2+}(300 \mathrm{mM})$, which resulted in an increase of thermostability and activity $\left(76.8^{\circ} \mathrm{C}\right.$ and $0.896 \mathrm{mg} / \mathrm{mL}$, respectively). Thr262 was mutated to the positively conserved lysine, to improve the salt-bridge formation, which is reported to contribute to thermal stabilization. The resulting triple mutation (S226P/R228S/T262K) also in the presence of $\mathrm{Ca}^{2+}(300 \mathrm{mM})$ demonstrated an activity increase but a lower $\mathrm{T}_{\mathrm{m}}\left(76.2^{\circ} \mathrm{C}\right)$ when compared to the double mutant. F106Y mutation appears to cause a steric hindrance of the additional hydroxy group with the catalytic residues, Ser176 and His254 [56].

Mutations on the residues around the catalytic triad, oxyanion hole and the $\mathrm{Ca}^{2+}$ binding sites were built to analyze the effects on the catalytic activity, allowing for a better understanding of the enzyme [137]. In this study, a three-dimensional model was developed based on the first reported structure SvCut190S226P, which was further mutated to SvCut190S226P/R228S ( $\left.\mathrm{k}_{\text {cat }}=27 \pm 0.2 \mathrm{~s}^{-1} ; \mathrm{K}_{\mathrm{M}}=0.089 \pm 0.001 \mathrm{mM}\right)$ with PBSA as substrate. The residues neighboring the oxyanion hole (Phe106, Thr107, Gln138, and Trp201) were mutated to alanine and several other amino acid residues. Overall, the resulting variants decreased the $\mathrm{k}_{\text {cat }}$ value, except for $\mathrm{Q} 138 \mathrm{D}$ variant (increased $\mathrm{k}_{\text {cat }}$ to $61 \pm 0.3 \mathrm{~s}^{-1}$ ), and increased affinity towards PBSA, except for W201A and Q138D [137]. Senga et al. [135] further mutated SvCut190S226P/R228S by deleting the C-terminal residues (Lys305/Leu306/Asn307). The variant resulted in a $\mathrm{k}_{\text {cat }}$ of $100 \mathrm{~s}^{-1}$ and a $\mathrm{K}_{\mathrm{M}}$ of $0.282 \mathrm{mM}$.

Using the same SvCut190S226P/R228S 3D model [137], Oda et al. [134] applied in-site mutagenesis to the residues involving the $\mathrm{Ca}^{2+}$ binding sites, using PBSA as substrate, as 3D modeling revealed similar behavior between PBSA and PET. When compared to other cutinases, SvCut190 lacks an amino acid in the $\beta 1-\beta 2$ loop (Site 1); therefore, a serine was introduced between Phe77 and Ala78 (78Ser). This amino acid addition remarkably enhanced the enzymatic activity $\left(k_{\mathrm{cat}}=151 \mathrm{~s}^{-1}\right)$; however, its expression was exceptionally low and the thermostability was slightly decreased. In Site 2, a disulfide bond was introduced (D250C-E296C), which significantly increased $\mathrm{T}_{\mathrm{m}}$ to $79^{\circ} \mathrm{C}$.

Hantani et al. [138] analyzed the catalytic reaction of SvCut190S226P/R228S and SvCut190S226P/R228S/Q138A/D250C-296C towards BHET. Kinetic parameters could not be calculated for the later variant due to low solubility of BHET. The final product of BHET degradation was MHET, with no further conversion to TPA. As for Site 3, many mutations 
were engineered being $\mathrm{N} 202 \mathrm{H}$ the variant with better resulting $\mathrm{k}_{\text {cat }}$ and thermostability. SvCut190S226P/R228S/Q138A/D250C-E296C/Q123H/N202H is the most thermostable variant of the enzyme determined to date $\left(\mathrm{T}_{\mathrm{m}}=85.7^{\circ} \mathrm{C}\right)$. In the presence of $2.5 \mathrm{mM}$ of $\mathrm{Ca}^{2+}$ the variant showed a decrease in the thermostability of the enzyme to $82.6{ }^{\circ} \mathrm{C}$ [134].

Kawai et al. [56] and Oda et al. [138] performed studies on PET hydrolysis, where different PET films were utilized as substrates. In both studies, SvCut190S226P/R228S showed the highest activity between 60 and $65^{\circ} \mathrm{C}$. The first group analyzed the degradation rate of PET-GF and PET-S by SvCut190S226P/R228S at $63^{\circ} \mathrm{C}$ for 3 days. The weight losses measured from the molar ratio of TPA included in PET and TPA produced from PET showed a degradation rate of approximately $10.9 \pm 1.5 \%$ and $26.2 \pm 0.6 \%$ for PET-GF and PET-S. The analyses by hybrid ion trap/time-of-flight mass spectrometry coupled with liquid chromatography (LC-IT-TOF-MS) and high-performance liquid chromatography (HPLC) detected TPA as the major peak, and no BHET was detected, presumably due to further hydrolysis to TPA [56]. Later, Oda et al. [134] studied the degradation rate products of PET-GF (6.3\% of crystallinity) by SvCut $190226 \mathrm{P} / \mathrm{R} 228 \mathrm{~S}$ and variants with the highest melting points at $65^{\circ} \mathrm{C}$ and $70{ }^{\circ} \mathrm{C}$, respectively, for 3 days. SvCut190226P/R228S showed a degradation rate of $16.0 \pm 1.4$ and expectedly the variants showed higher degradation rate at $70{ }^{\circ} \mathrm{C}$, being the variant with the highest thermostability (SvCut190S226P/R228S/Q138A/D250C$\mathrm{E} 296 \mathrm{C} / \mathrm{Q} 123 \mathrm{H} / \mathrm{N} 202 \mathrm{H})$ the variant with the highest degradation rate (33.6 \pm 3.0$)$, resulting in an increase by over $30 \%$, compared with that of SvCut 190 at $63^{\circ} \mathrm{C}$.

\subsubsection{Proposed Mechanism}

Kawabata et al. [137] proposed a catalytic mechanism through molecular docking of partial structure PET model and PBSA substrate with the variant S226P/R228S. His254 deprotonates Ser176, which interacts with the carbon of a carbonyl group by nucleophilic attack, thus splitting the ester bond and forming an acyl-enzyme intermediate. This intermediate is subjected to nucleophilic attack by a water molecule, which is deprotonated by His254, and the resulting proton is transferred to Ser225, creating the conditions for a new catalytic cycle.

\subsubsection{Future Perspectives}

SvCut190 has great potential as a PET biocatalyst due to high thermostability of the resulted variants. However, its activity is much too low for its usage in the plasticdegrading industry. Therefore, computational confirmation of the catalytic mechanism should be developed for a better understanding of the reaction.

\subsection{Thermobifida Genus Cutinase 1 and Cutinase 2 (Cut1 and Cut2)}

\subsubsection{Discovery}

Cutinase 1 (Cut1) and cutinase 2 (Cut2) are two enzymes from the Thermobifida genus species, which includes Thermobifida fusca, Thermobifida alba, and Thermobifida cellulosilytica. The enzymes are identical, differing in only 18 amino acid residues. While T. fusca and T. cellulosilytica are both thermophilic bacteria and can grow at temperatures up to $55^{\circ} \mathrm{C}$ and $45^{\circ} \mathrm{C}$, respectively, T. alba is a mesophilic bacterium that can only grow at temperatures up to $37^{\circ} \mathrm{C}$. All of these cutinases have the same number of amino acids (262), apart from Cut2 of Thermobifida fusca (261). These cutinases were firstly reported as PET biocatalysts by Herrero et al. [139] in 2011.

\subsubsection{Structure}

Cut1 from Thermobifida fusca DSM44342 (TfCut1 (DSM44342), aka Thf42_Cut1) has high similarity with Thermobifida fusca KW3 (TfCut2), aka ThfKW3_Cut2), differing only in four amino acids [140]. However, structure for TfCut1 is yet to be determined.

The Cut2 from Thermobifida fusca KW3 (TfCut2) was the first cutinase with a resolved crystallographic structure, determined by Roth et al. [141] in its free state with resolutions of $1.4 \AA$ and $1.55 \AA$ and complexed with the inhibitor phenylmethylsulfonyl fluoride (PMSF) 
with resolution of $1.44 \AA$ in 2013. All crystallographic structures known for TfCut2, TcCut2, and TcCut1 are summarized in Table 6.

Table 6. Structures of Cut1 and Cut2.

\begin{tabular}{|c|c|c|c|c|c|c|}
\hline PDB Code & Enzyme & Resolution (Å) & Ligand & Mutation & Year of Deposition & Ref. \\
\hline 4CG1 & TfCut2 & 1.4 & Free & - & 2013 & [141] \\
\hline 4CG2 & TfCut2 & 1.44 & PMSF & - & 2013 & {$[141]$} \\
\hline 4CG3 & TfCut2 & 4.55 & Free & - & 2013 & [141] \\
\hline 5LUK & TcCut2 & 1.45 & Free & $\mathrm{R} 28 \mathrm{~N} / \mathrm{A} 30 \mathrm{~V}$ & 2016 & [142] \\
\hline 5LUJ & TcCut2 & 2.2 & Free & - & 2016 & {$[142]$} \\
\hline 5LUL & TcCut2 & 1.9 & Free & R19S/R29N/A30V & 2016 & {$[142]$} \\
\hline 5LUI & TcCut1 & 1.5 & Free & - & 2016 & {$[142]$} \\
\hline
\end{tabular}

The enzyme adopts a classical $\alpha / \beta$-hydrolase fold with a central nine-stranded $\beta$ sheet enveloped by $11 \alpha$-helices on both sides. The catalytic triad is in a cleft on the surface of the enzyme and is comprised of Ser130, His208, and Asp176. The oxyanion hole is formed by Met131 and Tyr60. The residues Ala55 to Ala65, Leu175 to Pro180 and His208 to Pro211 form the active site [141]. Trp155 and Phe209 interact with the aromatic rings of the dimer PET model, 2PET.

A highly flexible region is located around the amino acids Arg245 to Gly247 and the disulfide bond Cys241-Cys259 is located near this region [141]. The relatively high melting point $\left(71.2 \pm 0.2{ }^{\circ} \mathrm{C}\right)$ may be explained by the presence of this disulfide bond and the optimized hydrogen bond network.

The crystallographic structures of Cut1 and Cut2 from Thermobifida cellulosilytica (TcCut1 and TcCut2, aka Thc_Cut1 and Thc_Cut2, respectively) in their free state were later determined by Ribitsch et al. [142], with resolutions of $1.5 \AA$ for TcCut1 (PDB: 5LUI) and 1.45, 2.2, and $1.9 \AA$ forTcCut2 (PDB: 5LUK, 5LUJ, and 5LUL, respectively) in 2016, as described in Table 6. Overall, it is identical to the previously reported TfCut2, apart from the number of $\alpha$-helices (11 vs. 10) that flanks the $\beta$-sheet. The catalytic triad and oxyanion hole of both cutinases are the same, namely Ser131, His209, Asp177, Tyr61, and Met132, respectively.

Cut1 from Thermobifida alba (TaCut1 aka Tha_Cut1) and TcCut1 have high similarity, differing in only four amino acids in the outside region of the active site [140]. However, no crystallographic structures have been determined so far.

\subsubsection{Activity}

Both Cut1 and Cut2 from the previously described organisms are able to degrade PET films. The major product released from the PET hydrolysis by TcCut1 and TfCut1 (DSM44342) was TPA [139]. However, MHET was the major product released by TcCut2, revealing a limiting rate on the MHET hydrolysis to TPA [139]. In addition, an inhibitory effect of BHET on PET hydrolysis by TcCut1 has been reported [143]. Activity of TcCut1 on semi-crystalline and amorphous PET films ( 24 and $12 \%$ crystallinity, respectively) demonstrated that the reaction is faster towards semi-crystalline PET [143].

TfCut2 also demonstrated low capability for MHET hydrolyzation to TPA [144-146]. Kinetic parameters of the hydrolysis of MHET and BHET substrates were measured by Barth et al. [144]. The low $\mathrm{k}_{\text {cat }}$ and high $\mathrm{K}_{\mathrm{M}}$ towards MHET $\left(0.31 \pm 0.01 \mathrm{~s}^{-1}\right.$ and $7.33 \times 10^{-3} \pm 3.62 \times 10^{-4} \mathrm{~mol} \mathrm{~L}^{-1}$, respectively) confirm the inhibitory effect of MHET. BHET also has inhibitory effects resulting in $\mathrm{k}_{\text {cat }}$ and $\mathrm{K}_{\mathrm{M}}$ of $26.76 \pm 0.85 \mathrm{~s}^{-1}$ and $3.97 \times 10^{-2} \pm 2.50 \times 10^{-3} \mathrm{~mol} \mathrm{~L}^{-1}$, respectively.

Wei et al. [145] demonstrated that when TfCut2 is complexed with the dimer PET model 2PET, the residues Gly62, Thr63, Ile178, and Ile213 become in close vicinity to the substrate. The studies of enzymatic degradation calculated with PET amorphous films weight loss revealed that the variant G62A had the highest weight loss of amorphous PET 
films $(42.6 \pm 2.9 \%)$, while the WT resulted in a weight loss of only $15.9 \pm 1.8 \%$. The variant I213S resulted in the highest thermal stability [145].

The substitution of Asp174, Asp204, and Glu253 with arginine confirmed their roles as residues related to the $\mathrm{Ca}^{2+}$ binding site in $T f C u t 2$ [114]. The resulting variants (D204A and E253A) increased $\mathrm{T}_{\mathrm{m}}$ values from $71.2 \pm 0.2{ }^{\circ} \mathrm{C}$ to $85.2 \pm 0.5^{\circ} \mathrm{C}$ and $86.2 \pm 0.8^{\circ} \mathrm{C}$. Substitution of the $\mathrm{Ca}^{2+}$ binding site residues of $\mathrm{TfCut} 2$ to replicate a disulfide bond was studied by Then et al. [147]. The resulting variant (D204C-E253C) increased the melting point to $92.8 \pm 0.3{ }^{\circ} \mathrm{C}$. Further engineered variants (D204C/E253C/D174R and D204C/E253C/D174R) increased the PET films weight loss (25.0 $\pm 0.8 \%$ and $24.2 \pm 0.8 \%$, respectively), when compared to WT TfCut2 $(16.3 \pm 2.2 \%)$. The other hydrolase from the same strain $\mathrm{TfCut1}(\mathrm{KW} 3)$ has a $\mathrm{T}_{\mathrm{m}}$ value of $68.6^{\circ} \mathrm{C} \pm 0.6^{\circ} \mathrm{C}$ and its thermostability increases in the presence of $\mathrm{Ca}^{2+}$ [114]. These mutations are summarized in Table S7.

TcCut1 and TcCut 2 differ in only 18 amino acids; however, the hydrolysis of PET films by TcCut1 was much more effective than that of TcCut2 [139]. Herrero et al. [139] observed electrostatic potential and hydrophobicity differences in two regions. The first region of TcCut1 is composed of Ser19, Asn29, Val30, and Glu65, whereas in TcCut2 it is composed of the positively charged Arg19 and Arg29, Ala30, and Gln65. The second region of TcCut1 is made up of Ala183 and Lys187, whereas in TcCut2 the residues are the more hydrophobic Leu183 and Arg187, as evidenced by Figure 14. In a later study, the group applied in-site mutagenesis in regions 1 and 2 of TcCut 2 to resemble TcCut1 [148]. The double mutant $\mathrm{R} 29 \mathrm{~N} / \mathrm{A} 30 \mathrm{~V}$ proved to be the most active variant, with a four-fold activity enhancement for amorphous PET films degradation.

TcCut1

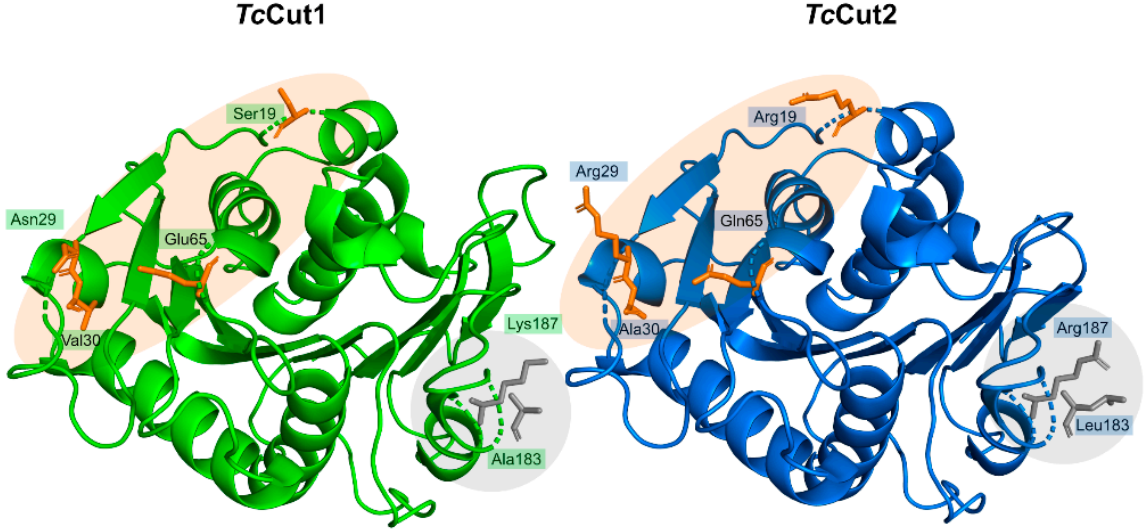

Figure 14. Comparison between different regions in TcCut1 (PDB: 5LUI), in green, and TcCut2 (PDB: 5LUJ), in blue. Region 1 and region 2 are represented in orange and grey, respectively. Amino acid residues are identified by their three-letter code.

The hydrolysis of a PET model bis(benzoyloxyethyl) terephthalate (3PET) by TaCut1, which has high similarity to TcCut1, resulted in a product release of mainly MHET and HEB, and also marginal amounts of TPA and BA [140]. Other methods for enhancement of enzymatic activity of TcCut1, such as fusion of binding domains, covalent fusion to hydrophobins, and ultrasound have also been reported [149-151].

\subsubsection{Future Perspectives}

The Cut1 and Cut2 of the Thermobifida genus have great potential as biocatalysts. A better understanding of the interaction between the PET substrate and the cutinases is needed for PET hydrolysis enhancement. Therefore, studies on the enzymatic mechanism and in-site mutagenesis should be developed. 


\subsection{Fusarium oxysporum Cutinase 5 (FoCut5a)}

\subsubsection{Discovery}

FoCut5a is a cutinase 5 from Fusarium oxysporum, an ascomycete fungus found mainly in soil [152]. FoCut5a was firstly expressed and characterized in 2015 by Dimarogona et al. [153], which confirmed FoCut5a ability to degrade PET into MHET.

\subsubsection{Structure}

The enzyme adopts the typical $\alpha / \beta$-hydrolase fold with a central $\beta$-sheet of 5 parallel $\beta$-strands and $11 \alpha$-helices, and has a molecular weight of $23 \mathrm{kDa}$ and 230 amino acids [153]. The catalytic triad is made up of Ser121, His189, and Asp176 (Figure 15A). The catalytic serine is located in the nucleophilic elbow, between strand $\beta 5$ and helix $\alpha 5$. References on the residues comprising the oxyanion hole are yet to be reported $[153,154]$, but an analysis of its X-ray structure suggests these residues are Ser43 and Gln123 (Figure 15A).

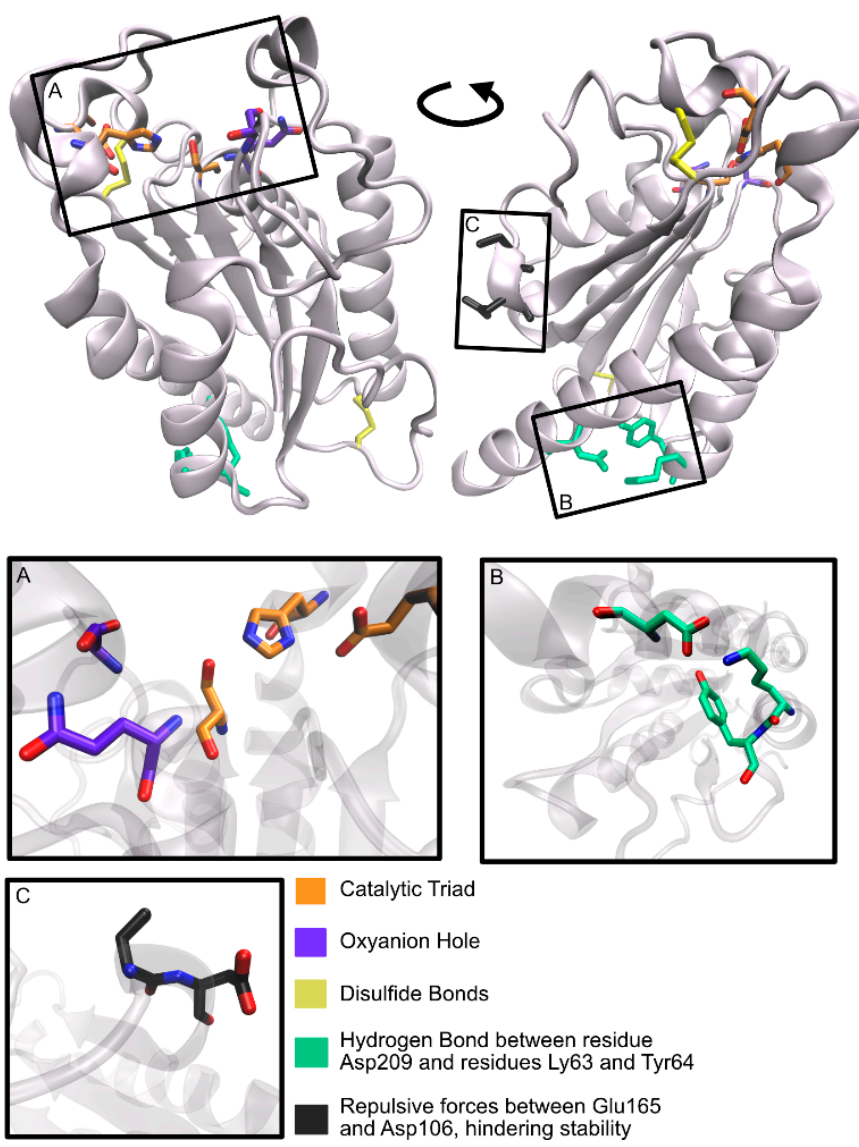

Figure 15. Schematic representation of main structural aspects of FoCut5a. (A) Detailed view of the catalytic triad and oxyanion hole residues. (B) Schematic representation of the hydrogen bond between residues Lys63 and Asp209, suspected to confer extra thermal stability to the enzyme. (C) View of the two repulsing amino acid residues Glu165 and Asp106, thought to hinder enzyme stability.

Two disulfide bonds are present in the enzyme. The first disulfide bond (Cys32Cys110) connects the loop $\alpha 1-\beta 1$ to loop $\alpha 4-\beta 3$ and the second (Cys172-Cys179) connects the loop $\beta 5-\alpha 8$ to helix $\alpha 8$, where the catalytic triad residue Asp176 is located $[153,154]$. The polar residues Lys63 and Tyr64, located at the end of helix $\alpha 2$, form a hydrogen bond with Asp209, which is in the helix $\alpha 11$ (Figure 15B). The interaction between the two is thought to increase the overall thermal stability of the enzyme. Furthermore, the helices $\alpha 10$ and $\alpha 11$ are bridged by the hydrogen bond between the amino acid Gly193 and the 
backbone oxygen of Leu190. Due to repulsive forces between the Glu165 and Asp166, the stability of the enzyme can be compromised [153] (Figure 15C).

So far, only one crystallographic structure of the enzyme has been reported, having been determined by Dimarogona et al. [153] in its free state with a resolution of $1.9 \AA$ (PDB: $5 \mathrm{AJH})$, in 2015.

\subsubsection{Activity}

The optimal parameters for FoCut5a activity are $40{ }^{\circ} \mathrm{C}, \mathrm{pH} 8$ and 1.92 mg enzyme loading per gram of PET fabric [153-155]. Nikolaivits et al. [154] studied the influence of different FoCut5a expression hosts on the formation of the disulfide bond and therefore the enzymatic thermostability. The expression of the cutinase gene in the oxidative cytoplasm of Origami 2 competent cells resulted in the highest thermostability, with a melting point of $44.9 \pm 0.5^{\circ} \mathrm{C}$.

This cutinase has been shown to hydrolyze the $p$ NP esters $p$ NPA, $p$ NPB, and $p$ NPL where the hydrolysis of $p$ NPB resulted in the highest catalytic activity and affinity $\left(\mathrm{k}_{\mathrm{cat}}=111.9 \pm 10 \mathrm{~s}^{-1}\right.$ and $\left.\mathrm{K}_{\mathrm{M}}=0.7 \pm 0.2 \mathrm{mM}\right)$ [153].

PET hydrolytic activity was confirmed when model 3PET was used as a substrate [153]. FoCut5a was able to hydrolyze the model substrate, being the products released TPA, BHET and benzoic acid (BA) $(0.19,0.20$ and $1.09 \mathrm{mM}$, respectively). The enzyme was confirmed to further hydrolyze BHET to MHET, when BHET was used solely as the substrate, resulting in a $46 \%$ abundance of MHET. The cutinase successfully depolymerized PET fabrics, resulting in a release of $26 \mu \mathrm{M}$ TPA and derivatives [153]. The application of in-site mutagenesis studies is yet to be reported.

\subsubsection{Future Perspectives}

FoCut5a is a promising enzyme for PET hydrolysis application, albeit, determination of the X-ray structure complexed with PET substrate should be attempted to enable a better understanding of the key residues involved in the reaction and the catalytic mechanism. Insite mutagenesis could also be applied for enhancement of both activity and thermostability.

\subsection{Humicola insolens Cutinase (HiCut)}

\subsubsection{Discovery}

$\mathrm{HiCut}$ aka $\mathrm{HiC}$, is a cutinase produced by Humicola insolens, a thermophilic fungus capable of growing at temperatures up to $58^{\circ} \mathrm{C}$ [156], first reported in 1964. HiCut ability to degrade PET substrates was first reported by Ronkvist et al. [157] in 2009. Castro et al. [158] determined that $\mathrm{HiCut}$ has the highest activity towards PET bottle when compared to other 15 biocatalysts, being the amount of released products 29 times higher than the second-best enzyme, RoLip (commonly known has RoL).

\subsubsection{Structure}

The enzyme presents an $\alpha / \beta$-fold with a central five-stranded $\beta$-sheet, which is surrounded by six $\alpha$-helices on both sides. The enzyme has a molecular weight of $32 \mathrm{kDa}$ and 194 amino acids. The central $\beta$-sheet is well conserved in the lipase-cutinase family [159]. The conserved catalytic triad is composed of Ser105, His173, and Asp160 [159]. In the hydrophobic loops adjacent to the active site, three amino acid residues (Leu66, Leu167, and Ile169) may be restricting the entrance of larger substrate [160].

According to UniProtKB [161], HiCut also exhibits two disulfide bonds (Cys17Cys94 and Cys156-Cys163). References on the residues comprising the oxyanion hole are yet to be reported, but upon visual inspection of the X-ray structure, residues Gln106 and Ser28 likely make up this motif. The two structures available for HiCut are described in Table 7. The enzyme in its free form (resolution of $3 \AA$, PDB: 4OYY) and in complex with mono-ethyl phosphate (resolution of $2.05 \AA$ A PDB: 4OYL), determined by Kold et al. [159], in 2014. 
Table 7. Crystallographic structures of HiCut.

\begin{tabular}{cccccc}
\hline PDB Code & Resolution $(\AA)$ & Ligand & Mutation & Year of Deposition & Ref. \\
\hline 4 OYY & 3 & - & - & 2014 & {$[159]$} \\
\hline 4 OYL & 2.05 & Mono-ethyl phosphate & - & 2014 & {$[159]$} \\
\hline
\end{tabular}

\subsubsection{Activity}

The enzyme has a relatively high thermostability, showing maximum initial activity at $80^{\circ} \mathrm{C}$ and $\mathrm{pH} 8.5$ and becomes totally inactive at $90^{\circ} \mathrm{C}$, presumably due to thermal-induced denaturation [157]. However, Hunsen et al. [162] and Fabbri et al. [163] suggest an optimal temperature of $65-70{ }^{\circ} \mathrm{C}$. High selectivity of $\mathrm{HiCut}$ 's towards long-chain alcohols and acids was revealed, with conversion rates up to $90 \%$ when using a 12-chain alcohol, 60 to $70 \%$ when using an 8 carbon-chain acid and only $30 \%$ when using small acid chains [163,164].

Ronkvist et al. [157] determined that HiCut fully catalyses low crystallinity PET (7\% crystallinity) to TPA at $70{ }^{\circ} \mathrm{C}$, resulting in a $97 \pm 3 \%$ weight loss in $96 \mathrm{~h}$. However, Castro et al. [158] and Carniel et al. [165] determined a limiting rate on the MHET hydrolysis to TPA when PET and its intermediate BHET were used as substrates. A combination of $\mathrm{HiCut}$ with an enzyme that can properly hydrolyze MHET into TPA ( $\mathrm{C} a \mathrm{LipB})$ was therefore implemented, resulting in a 7.7-fold increase of TPA concentration [165]. Expectedly, HiCut reveals a decrease in activity when exposed to higher crystallinity PET films, as determined by Ronkvist et al. [157], where HiCut activity decreased 10-fold when exposed to PET film with $35 \%$ crystallinity compared to the PET films with $7 \%$ crystallinity.

While studying the enzymatic hydrolysis of two phthalic acid based polyester coatings (PTa and PTb) by HiCut and TlLip, Greimel et al. [166] determined that only the cutinase from Humicola insolens was able to hydrolyze the substrates. The unexpected inactivity by TlLip indicates that the ester bonds between TPA and EG are more accessible to enzymatic hydrolysis than ester bonds between phthalic acid and trimethylolpropane (like in PTa and $\mathrm{PTb})$.

Quartinello et al. [167] developed a two-step system, involving depolymerization and enzymatic hydrolysis. The first step is the depolymerization of the virgin PET fiber, where the optimal conditions were set to be $250{ }^{\circ} \mathrm{C}$ and 39 bar. The process completely reduced the substrate to powder consisted of $85 \%$ TPA and small PET oligomers. The second step is the introduction of a catalytic enzyme ( $\mathrm{HiCut)}$ to further hydrolyze the PET oligomers, which resulted in a total of $97 \%$ TPA production.

In addition to temperature and $\mathrm{pH}$, other conditions were shown to increase the hydrolysis product concentrations. Carniel et al. [168] studied the hydrolysis reaction of post-consumer PET by HiCut and determined that the unbuffered reaction (alkaline water $\mathrm{pH}$ 8.95) with $\mathrm{NaOH} 0.5 \mathrm{M}$ to control the reaction $\mathrm{pH}$ showed a 2.39-fold improvement in the released monomers when compared to the Tris-HCl-buffered ( $\mathrm{pH} 8.95$ ). The temperature was constant for both reactions $\left(50^{\circ} \mathrm{C}\right)$. Eugenio et al. [169] determined that the best fitting enzymatic concentration for optimal reaction rate is about $1.68 \mathrm{mg}_{\text {protrein }} \mathrm{mL}^{-1}$.

Many other methodologies that lead to increased enzymatic activity are reported, including DNA immobilization [170] and optimization of process variables [171]. No studies regarding enzymatic activity and thermostability enhancement towards PET substrate with application of in-site mutagenesis are yet available. However, variants of the enzyme are reported to improve the enzymatic activity of cellulose acetate deacetylation and polyacrylates and poly(vinyl acetate) hydrolase [172,173].

\subsubsection{Future Perspectives}

Although HiCut has promising results for PET degradation, due to higher thermostability and activity when compared to other enzymes, it is still far away from being usable in a large scale. Even though no mechanistic proposals for HiCut degradation of PET-like substrates is available, the existence of a three-dimensional structure complexed with a ligand provides an important starting point for molecular docking and molecular dynamics 
simulation studies. Studies on the key residues involved in the reaction should be developed for a better understanding of the catalytic mechanism. To increase the enzymatic activity, studies with in-site mutagenesis in these residues should be applied. Kinetic parameters such as $\mathrm{k}_{\mathrm{cat}}$ and $\mathrm{K}_{\mathrm{M}}$ should be calculated.

\subsection{Fusarium solani Cutinase ( $F$ s Cut)}

\subsubsection{Discovery}

FsCut or FsC, Fusarium solani cutinase, is a fungal cutinase [174] and type I PET hydrolase. This fungus is a widely spread soil pathogen [175]. It was first characterized by Lin et al. [176] in 1980, and several structural and biochemical studies have followed.

\subsubsection{Structure}

Fs Cut is a 230 amino acid residue protein with a molecular weight of $23 \mathrm{kDa}$. The enzyme is a compact functional monomer with a typical $\alpha / \beta$-serine hydrolase overall fold. It is made up of a central $\beta$-sheet with five parallel $\beta$-strands and five $\alpha$-helices on either side $[174,177,178]$. The residues Ser120, His188 and Asp175 make up the catalytic triad [174], stabilized by oxyanion hole residues Gln121 and Ser42 [177]. Catalytic serine is inserted in a Gly-Tyr-Ser-Gln-Gly motif in a $\beta$-hairpin [174].

The extended binding site is mostly hydrophobic, but solvent accessible. It is partly covered by two thin "bridges", formed by amino acid residues Leu81, Val184, Leu182, and Asn84 [174,177,178]. A conserved water molecule bound to Leu179, Ala185, Ile183, and catalytic residue Asp175 has a role in properly orienting side-chains and facilitating catalysis [177]. Binding site amino acids have overall higher B factor than the remaining protein residues, suggesting conformation changes and rearrangements upon binding and catalytic attack [174].

The 46 three-dimensional structures of Fs Cut available on PDB are summarized in Table 8. There are currently five apo-form and five complexed WT structures available, and thirty-six engineered variants. The first structure was deposited by Martinez et al. [174] in 1994, at $1.25 \AA$ (PDB: 2CUS). In the same year, a structure complexed with a covalent inhibitor (PDB: 2CUT) revealed the displacement of a conserved water molecule upon binding, with no other structural aspects affected [177]. Longhi et al. [179] produced 31 FsCut variants with different mutated residues and complexed ligands, and studied the effects of these alterations on the enzymatic structure, having concluded that the binding of a non-hydrolysable substrate analogue did not induce structural rearrangements. The same authors [180] later obtained a complex of $F s C u t$ with a triglyceride analogue similar to the first tetrahedral intermediate (TI) in the reaction pathway (PDB: 1CEX), further confirming the unaffected overall structural and catalytic fold.

Table 8. Crystallographic structures of FsCut.

\begin{tabular}{|c|c|c|c|c|c|}
\hline PDB Code & Resolution (Å) & Ligand & Mutations & Year of Deposition & Ref. \\
\hline 1CUS & 1.25 & - & - & 1994 & {$[174]$} \\
\hline $2 \mathrm{CUT}$ & 1.90 & Diethyl Phosphonate & - & 1994 & [177] \\
\hline 1FFA & 1.69 & - & N84A & 1995 & {$[178]$} \\
\hline $1 \mathrm{FFB}$ & 1.75 & - & N84D & 1995 & [178] \\
\hline 1FFC & 1.75 & - & N84L & 1995 & {$[178]$} \\
\hline $1 F F D$ & 1.69 & - & N84W & 1995 & {$[178]$} \\
\hline 1FFE & 1.69 & - & S42A & 1995 & {$[178]$} \\
\hline $1 X Z A$ & 1.80 & - & S129C & 1995 & [179] \\
\hline $1 \mathrm{XZB}$ & 1.75 & Mercury Acetate & S129C & 1995 & {$[179]$} \\
\hline $1 X Z C$ & 1.75 & Para-Sulfurous phenyl mercury & S129C & 1995 & {$[179]$} \\
\hline
\end{tabular}


Table 8. Cont.

\begin{tabular}{|c|c|c|c|c|c|}
\hline PDB Code & Resolution (Å) & Ligand & Mutations & Year of Deposition & Ref. \\
\hline 1XZD & 2.70 & - & S213C & 1995 & [179] \\
\hline $1 \mathrm{XZE}$ & 1.75 & - & S92C & 1995 & [179] \\
\hline $1 \mathrm{XZF}$ & 1.69 & - & T144C & 1995 & [179] \\
\hline $1 X Z G$ & 1.69 & - & $\mathrm{T} 45 \mathrm{~A}$ & 1995 & [179] \\
\hline $1 \mathrm{XZH}$ & 1.69 & - & T80P & 1995 & [179] \\
\hline 1XZI & 1.69 & - & $\mathrm{Y} 119 \mathrm{H}$ & 1995 & [179] \\
\hline 1XZJ & 1.69 & - & $\mathrm{T} 38 \mathrm{~F}$ & 1995 & [179] \\
\hline 1CUA & 1.80 & - & N172K & 1995 & [179] \\
\hline $1 \mathrm{CUB}$ & 1.75 & - & N172K/R196D & 1995 & [179] \\
\hline 1CUC & 1.75 & - & N172K/R196D & 1995 & [179] \\
\hline 1CUD & 2.70 & - & N172K/R196D & 1995 & [179] \\
\hline 1CUE & 2.10 & - & Q121L & 1995 & [179] \\
\hline $1 \mathrm{CUF}$ & 1.75 & - & R156L & 1995 & [179] \\
\hline $1 C U G$ & 1.75 & - & R17E/N172K & 1995 & [179] \\
\hline $1 \mathrm{CUH}$ & 1.75 & - & R196E & 1995 & [179] \\
\hline 1CUI & 2.50 & - & S120A & 1995 & [179] \\
\hline $1 \mathrm{CUJ}$ & 1.60 & - & S120C & 1995 & [179] \\
\hline $1 \mathrm{CUU}$ & 1.69 & - & A199C & 1995 & [179] \\
\hline $1 C U V$ & 2.01 & - & A85F & 1995 & [179] \\
\hline $1 \mathrm{CUW}$ & 2.70 & - & $\begin{array}{l}\text { G82A/A85F/ } \\
\text { V184I/L189F }\end{array}$ & 1995 & [179] \\
\hline 1CUX & 1.75 & - & L114Y & 1995 & [179] \\
\hline 1CUY & 1.69 & - & L189F & 1995 & [179] \\
\hline $1 \mathrm{CUZ}$ & 2.10 & - & L81G/L182G & 1995 & [179] \\
\hline 1XZK & 2.01 & & - & 1995 & [179] \\
\hline 1XZL & 1.69 & N-hexylphosphonate ethyl ester & - & 1995 & [179] \\
\hline $1 \mathrm{XZM}$ & 1.75 & $\begin{array}{c}\text { N-undecyl o-methyl chloro } \\
\text { Phosphonate ester }\end{array}$ & - & 1995 & [179] \\
\hline $10 \times M$ & 2.30 & $\begin{array}{c}\text { triglyceride analogue } \\
\text { ([(2R)-2-(butylcarbamoyloxy)-3- } \\
\text { butylphosphonoyloxypropyl] } \\
N \text {-butylcarbamate) }\end{array}$ & - & 1996 & [180] \\
\hline 1AGY & 1.15 & - & - & 1997 & [181] \\
\hline 1CEX & 1.00 & - & - & 1997 & [181] \\
\hline 3EF3 & 1.50 & - & N172K & 2008 & [182] \\
\hline $3 E S A$ & 2.00 & - & N172K & 2008 & [182] \\
\hline 3ESB & 2.30 & - & N172K & 2008 & [182] \\
\hline 3ESC & 1.20 & $\begin{array}{l}\text { triglyceride analogue (ethyl } \\
\text { 4-nitrophenyl } \\
\text { P-[3-(4-(bromopallado)-1,3- } \\
\text { bis[(methylthio)methyl]- } \\
\text { phenyl)propyl]phosphonate) }\end{array}$ & N172K & 2008 & [182] \\
\hline
\end{tabular}


Table 8. Cont.

\begin{tabular}{cccccc}
\hline PDB Code & Resolution $(\AA)$ & Ligand & Mutations & Year of Deposition & Ref. \\
\hline 3ESD & 1.22 & $\begin{array}{c}\text { triglyceride analogue (ethyl } \\
\text { 4-nitrophenyl } \\
\text { P-[3-(4-(bromopallado)-1,3- } \\
\text { bis[(methylthio)methyl]- } \\
\text { phenyl)propyl]phosphonate) }\end{array}$ & N172K & 2008 & [182] \\
\hline 3QPA & 0.85 & - & - & 2011 & N/A \\
\hline 3QPC & 0.98 & - & - & N/A \\
\hline
\end{tabular}

\subsubsection{Activity}

Heumann et al. [183] have demonstrated FsCut's ability to hydrolyze BHET, and to modify the surface of synthetic PET fabric with positive yields.

In order to enhance enzymatic activity towards PET, Araújo et al. [184] tested several active site point mutations that aimed to enlarge the binding site, providing a better fit for larger polymer chains. Through computational studies of molecular dynamic simulations and free energy calculations with a substrate mimicking the TI, five point mutations were predicted as favorable for activity and model TI stabilization-L182A, V184A, L189A, L81A, and N84A. Experimental testing of these variants with PET fabric as a substrate for $48 \mathrm{~h}$ at $\mathrm{pH} 7.5$ and $37^{\circ} \mathrm{C}$ showed L182A, L81A, and V184A resulted in increased activity, while L189A and N84A resulted in activity decreases. Subsequent structural and hydrophobicity studies confirmed that the better performing variants resulted in a less restrained active site, allowing for better accommodation of substrate and higher stabilization of TI, without affecting the hydrophobicity and therefore the grip on PET fabric surface.

Ronkvist et al. [157] studied the effect of PET crystallinity percentage on cutinase activity and compared FsCut with $\mathrm{HiCut}$ and $\mathrm{PmCut}$. The substrates were two samples of PET-low crystallinity PET (7\%) and a bi-oriented sample of PET with $35 \%$ crystallinity. Of the three enzymes tested, FsCut showed the lowest affinity for PET and the poorer thermal stability, with a maximum activity at $50{ }^{\circ} \mathrm{C}$, far from PET $\mathrm{T}_{\mathrm{g}}$. FsCut ability to degrade PET decreased as sample crystallinity increased, as expected. The mild temperature for FsCut maximum activity combined with its rapid denaturation as temperature increases constitutes a meaningful impairment to its PET degrading action.

Erbel et al. [185] tested FsCut activity on PET fabrics and 3PET in the presence of Triton X-100 and plasticizer N,N-diethyl-2-phenylacetamide (DEPA). Plasticizers enhance polymer chain mobility by reducing $\mathrm{T}_{\mathrm{g}}$ and inner chain interaction. Even though Triton- $X-$ 100 had no effect on FsCut ability to modify PET, DEPA resulted in enhanced hydrolysis rates, further confirming that FsC biggest limitation in degrading PET is the polymer's high $\mathrm{T}_{\mathrm{g}}$ and low chain mobility at the enzyme's optimal temperature.

\subsubsection{Future Perspectives}

Fs Cut, similar to many known and described cutinases, is regarded as a promising PET hydrolase. However, thermal stability and low optimal activity is a main issue in the application of this enzyme for efficient PET depolymerization. There have been no mechanistic proposals for the FsCut degradation mechanism of PET. Since the structure and activity of this enzyme is extensively characterized, several computational studies could be confidently employed to resolve the enzymatic mechanism, particularly QM/MM [102-104]. The existence of high-resolution three-dimensional structures with a TI model substrate is a relevant clue for mechanistic studies and could be used to validate a QM/MM study. Finally, strategies to improve thermal stability should be employed. 


\subsection{Candida antarctica Lipase B (CaLipB)}

\subsubsection{Discovery}

CaLipB, CalB, or lipase B from Candida antarctica, was first isolated in 1988 [186,187], and has been the target of various studies. CaLipB is a multi-purpose enzyme, with various roles attributed to it, such as resolution of alcohols [188] and amines, desymmetrization of diacetates and diols, synthesis of intermediates for substrate, and pharmaceuticals production [189]. This large number of applications arises because $C a$ LipB presents a high thermal stability, low substrate specificity, high enantioselectivity, and stability in diverse solvents [190].

\subsubsection{Structure}

CaLipB is made up of 317 amino acid residues and has a molecular weight of $33 \mathrm{kDa}$. Optimal catalytic $\mathrm{pH}$ was determined to be 7 . In addition, $\mathrm{CaLipB}$ was shown to remain stable in aqueous media from $\mathrm{pH} 3.5$ to 9.5 [191]. The overall structure follows the typical $\alpha / \beta$-globular protein fold. The catalytic site contains the typical Ser-His-Asp triad (Ser105, His224, Asp187), stabilized by an oxyanion hole (Thr40 and Gln106). Ser105 is introduced in a TWSQG motif, differing from the typical motif characteristic of PET hydrolyzing enzymes [192]. CaLipB exists as a monomer, mostly composed of $\beta$-sheets surrounded by $\alpha$-helices, similar to the typical $\alpha / \beta$-serine hydrolase fold $[188,192]$.

The catalytic triad is located at the carboxy-terminal hedge of the main parallel $\beta$ sheet, with Ser105 located in a tight turn between $\alpha 4$ and $\beta 4$. His224, located in helix $\alpha 9$ with a protruding sidechain into the active site, is at optimal catalytic distance from Asp187, located in a turn after the sixth strand. Ser105 is surrounded by polar residues (Thr40, Asp134, Gln157) that form hydrogen bonds and are fully accessible to solvent interactions [192]. Stauch et al. [191] revealed the existence of a conserved water molecule shared between catalytic His224 and Ser105, while Strzelczyk et al. [189] identified a water bound to catalytic Asp187 and nearby Ser227. The binding pocket is divided into two compartments with different affinities-one that accommodates the acyl moiety and the other that preferably binds the substrate alcohol part [188,189,192]. The alcohol-binding subsite is further divided into a large and a medium pocket [193].

There are currently $25 \mathrm{CaLipB}$ three-dimensional structures in the Protein Data Bank, including apo forms of the enzyme and forms co-crystallized with ligands of relevance. These are summarized in Table 9.

Table 9. Crystallographic structures of CaLipB.

\begin{tabular}{|c|c|c|c|c|c|}
\hline PDB Code & Resolution (Å) & Ligand & Mutations & Year of Deposition & Ref. \\
\hline $1 \mathrm{TCA}$ & 1.55 & - & - & 1994 & [192] \\
\hline $1 \mathrm{TCB}$ & 2.10 & - & - & 1994 & [192] \\
\hline 1TCC & 2.5 & - & - & 1994 & [192] \\
\hline 1LBS & 2.60 & Phosphonate inhibitor & - & 1995 & [188] \\
\hline 1LBT & 2.50 & Tween 80 & - & 1995 & [188] \\
\hline $3 \mathrm{ICV}$ & 1.49 & - & - & 2009 & [194] \\
\hline $3 \mathrm{ICW}$ & 1.69 & $\begin{array}{l}\text { Inhibitor (methyl hydrogen } \\
\text { (R)-hexylphosphonate) }\end{array}$ & - & 2009 & [194] \\
\hline 3W9B & 2.90 & - & - & 2013 & $\mathrm{~N} / \mathrm{A}$ \\
\hline $4 \mathrm{~K} 5 \mathrm{Q}$ & 1.49 & - & D223G/L278M & 2013 & [195] \\
\hline $4 \mathrm{~K} 6 \mathrm{~K}$ & 1.60 & - & D223G & 2013 & [195] \\
\hline $4 \mathrm{~K} 6 \mathrm{H}$ & 1.60 & - & L278M & 2013 & [195] \\
\hline $4 \mathrm{~K} 6 \mathrm{G}$ & 1.50 & - & - & 2013 & [195] \\
\hline $4 \mathrm{ZV7}$ & 2.00 & - & - & 2015 & [195] \\
\hline
\end{tabular}


Table 9. Cont.

\begin{tabular}{|c|c|c|c|c|c|}
\hline PDB Code & Resolution ( $\AA$ ) & Ligand & Mutations & Year of Deposition & Ref. \\
\hline $5 \mathrm{~A} 6 \mathrm{~V}$ & 2.28 & - & - & 2015 & [191] \\
\hline $5 \mathrm{~A} 71$ & 0.91 & - & - & 2015 & [191] \\
\hline 5GV5 & 2.89 & - & - & 2016 & [193] \\
\hline 6ISP & 1.88 & - & W104V/A281Y/A282Y/V149G & 2018 & [196] \\
\hline 6ISQ & 1.86 & - & W104V/S105C/A281Y/A282Y/V149G & 2018 & [196] \\
\hline 6ISR & 2.6 & - & W104V/S105C/A281Y/A282Y/V149G & 2018 & [196] \\
\hline $6 \mathrm{~J} 1 \mathrm{P}$ & 1.76 & - & A281G/A282V/V190C & 2018 & [197] \\
\hline $6 \mathrm{~J} 1 \mathrm{R}$ & 1.6 & - & Q157L/I189A & 2018 & [197] \\
\hline $6 \mathrm{~J} 1 \mathrm{Q}$ & 1.6 & - & W104A/I189V & 2018 & [197] \\
\hline $6 \mathrm{~J} 1 \mathrm{~T}$ & 1.78 & $\begin{array}{l}\text { Synthesized product 3a' } \\
\text { ((2S)-2-phenyl-N-[(1R)-1- } \\
\text { phenylethyl]propanamide) }\end{array}$ & A281G/A282V/V190C & 2018 & [197] \\
\hline $6 \mathrm{~J} 1 \mathrm{~S}$ & 1.83 & - & W104A/I189M/V190C/D134L & 2018 & [197] \\
\hline $6 \mathrm{TP} 8$ & 1.55 & - & - & 2019 & [198] \\
\hline
\end{tabular}

Uppenberg et al. [188,192] solved the first X-ray structures of this enzyme in 1992. These structures revealed that all six cysteine residues in the protein form disulfide bridges: Cys22-Cys64 and Cys216-Cys258 contribute to the overall protein fold, and Cys293Cys311 stabilizes the enzymatic C-terminal. A possible N-glycosylation site at Asn74 was also identified.

From the resolved structures, amino acid sequence, and similarity to other well categorized lipases, the existence of a lid domain responsible for an open and closed conformations was predicted [192]. However, the proven existence of a lid and a precise characterization of it was only achieved in 2016, when Stauch et al. [191] resolved a twomonomer structure representing both conformations at $0.91 \AA$ (PDB: 5A71). The two monomers, $\mathrm{A}$ and $\mathrm{B}$, assume different conformations. Monomer A takes on the classical open conformation, similar to previously reported structures, while monomer B reveals a huge conformational change. Residue range Leu140-Leu147 is an $\alpha$-helix $(\alpha 5)$ in monomer $\mathrm{A}$, but in monomer B, it becomes an unfolded loop, corresponding to a closed conformation. The closing of the lid hinders substrate accessibility to the binding site, preventing catalytic activity.

Regarding the binding mode, Uppenberg et al. [192] obtained CaLipB in complex with Tween 80 (PDB: 1LBT), a monoester prior to hydrolysis, which remained highly exposed to solvent and did not disrupt the overall fold; and a complex with a phosphonate inhibitor (PDB: 1LBS), which revealed disruption of the optimal distances for catalysis. Qian et al. [194] solved a suicide-inhibitor-complexed structure after circular permutation, and Xu et al. [197] resolved a complex structure with a synthesized stereoisomer product in order to understand stereoselectivity of CaLipB.

\subsubsection{Activity}

CaLipB activity towards PET is not consensual $[51,199,200]$. The most promising report was the evidence found by Carniel et al. [165] that CaLipB has MHETase and BHETase activity, efficiently degrading BHET and MHET to TPA and EG. These studies were conducted synergistically with HiCut (as represented in Figure 16), which showed high affinity and activity in PET depolymerization. Using BHET as a substrate, Carniel et al. [165] screened 10 commercial enzymes and identified $\mathrm{CaLipB}$ and $\mathrm{HiCut}$ as potential biocatalysts. Preliminary screening was conducted at $\mathrm{pH} 7.0$ and $37^{\circ} \mathrm{C}$ and revealed that CaLipB led to a fast conversion of BHET to MHET and, consequently, MHET to TPA with higher extent than previously tested lipases. Activity assays with PET bottle after different pre-treatments showed a better performance achieved by HiCut than CaLipB. After 3 weeks of activity on the PET bottle and pellet, a maximum of $0.4 \%$ weight loss, but almost total 
degradation of MHET and BHET to TPA, was achieved when the reaction environment was high on these substrates. Therefore, further studies to enhance CaLipB activity as a promising MHET and BHET hydrolyzing enzyme are essential to confirm this enzyme as an essential player in the PET bioremediation landscape. To our knowledge, there are no reported studies aiming at enhancing CaLipB hydrolysis of PET, MHET, and BHET.

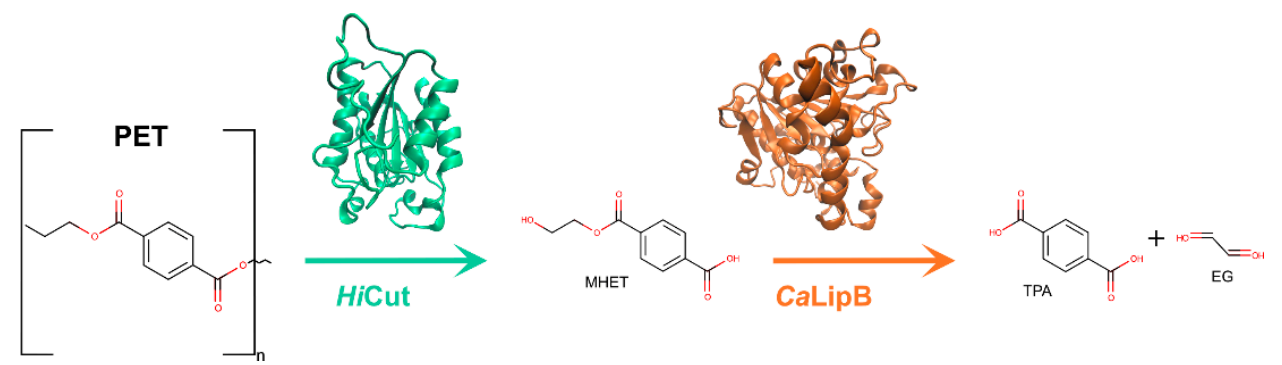

Figure 16. Cumulative action of HiCut and CaLipB to fully degrade PET to TPA and EG.

Due to the diverse applications of $\mathrm{CaLipB}$, countless engineering efforts towards increased activity and higher stability have been reported with various substrates, unrelated to PET or plastic polymers in general [201-207]. The ability of CaLipB to play an essential role in polyester and bioplastic synthesis has also been the target of interesting activity and catalytic studies [208,209].

Even though these studies are not specific to PET, some may result in valuable suggestions and strategies. Qian et al. [194] redesigned CaLipB by circular permutation, a technique that changes connectivity and sequence order but does not affect overall fold. Circular permutation occurs naturally throughout organism evolution and is frequently used as an artificial engineering technique. Variant CalB-cp283 was generated and its three-dimensional structure solved (PDB: 3ICV). Activity assays showed increased activity and a high-rate enhancement against $p$ NP-butyrate, and structural studies suggest CalBcp283 suffered dimerization. Xie et al. [195] followed the rationale of mutating residues within $10 \AA$ and a high B factor and obtained a double mutant D223G/L278M (PDB: 4K5Q) with higher activity against $p \mathrm{NP}$-caprylate and higher stability than the WT protein.

\subsubsection{Future Perspectives}

The recent discovery that $\mathrm{CaLipB}$ has degradation activity towards BHET and MHET and can act synergistically with PET degrading enzymes that are unable to complete degradation (such as IsPETase and HiCut), is a very promising approach. However, so far, no significant additional studies have been conducted. Given the high number of three-dimensional $\mathrm{CaLipB}$ structures, many of them recent and with high-resolution values, computational mechanistic studies aimed at understanding $\mathrm{CaLipB}$ as probable (yet disputed) role as a PET degrading enzyme could be conducted. Specific understanding of the binding mode of these molecules and mechanistic solving and characterization would allow for the development of engineered variants with enhanced activity. CaLipB has received little attention from the community as a PET degrading enzyme, but its promising experimental behavior justifies further investment.

\subsection{Thermobifida alba Esterase 1 (TaEst1)}

TaEst1 is a 260 amino acid residues cutinase from Thermobifida alba identified in 2013 [45,210]. TaEst1 has an increased activity on $p$ NPB of two-fold in comparison with to TaEst119 and is able to fully depolymerize 3PET to TPA and hydrolase the surface of PET film $[45,210]$.

Efforts to obtain a three-dimensional structure of TaEst1 have been reported by Kitadokoro et al. [211], but no resolved structure has been deposited on the PDB database to date. Thumarat et al. [210] built a homology model based on a known structure of TaEst119, and identified the catalytic triad as residues Ser169, His247, and Asp215 and the oxyanion 
hole as made up of Met170 and Tyr99, similar to TaEst119. The two enzymes differ in the residues of a loop near the substrate binding-site, which might explain the higher activity of TaEst1 compared with TaEst119. The overall fold is consistent with the typical $\alpha / \beta$-serine hydrolase structure, with a central, nine-stranded parallel $\beta$-sheet flanked by eight $\alpha$-helices on either side.

Kitadokoro et al. [211] determined the $\mathrm{T}_{\mathrm{m}}$ of $\mathrm{TaEst} 1$ to be $61^{\circ} \mathrm{C}$. Activity assays with $p \mathrm{NPB}$ in the presence and absence of $\mathrm{Ca}^{2+}$ cations at $\mathrm{pH} 6.0$ and $50^{\circ} \mathrm{C}$ revealed that in the presence of calcium ions $\mathrm{T}_{\mathrm{m}}$ increased up to $76^{\circ} \mathrm{C}$. Inspired by a conserved valine residue in most cutinases, variant $\mathrm{A} 68 \mathrm{~V}$ was tested and resulted in higher activity.

Thumarat et al. [210] engineered several TaEst1 variants and tested them for activity on $p \mathrm{NPB}$ substrate, as described in Table S8. The better performing variant (A68V/T253P) was later tested with 3PET and PET film as substrates. Variants with point mutations and combined modified residues A68V, T253P, and M256K were constructed based on typical cutinase residues absent in TaEst1. Variant A68V resulted in increased activity (similar to what had been observed by Kitadokoro et al. [211], but no increase in $\mathrm{T}_{\mathrm{m}}$. Therefore, variant $\mathrm{A} 68 \mathrm{~V} / \mathrm{T} 253 \mathrm{P}$ was constructed since proline residues typically stabilize overall enzymatic structure. This double mutant resulted in higher activity and an increased $\mathrm{T}_{\mathrm{m}}$ of $79^{\circ} \mathrm{C}$ in the presence of calcium. Furthermore, TaEst $1 \mathrm{~A} 68 \mathrm{~V} / \mathrm{T} 253 \mathrm{P}$ conserved $100 \%$ of activity at $50-55{ }^{\circ} \mathrm{C}$ after $1 \mathrm{~h}, 90 \%$ of activity at $60{ }^{\circ} \mathrm{C}$ for $1 \mathrm{~h}$ and $70 \%$ activity at $65^{\circ} \mathrm{C}$ for $30 \mathrm{~min}$. Therefore, this variant showed remarkably improved activity and thermal stability when compared with WT TaEst1. Variants A68V/M259K and A68V/T253P/M259K resulted in similar activity to the A68V/T253P double mutant but lower $\mathrm{T}_{\mathrm{m}}$.

Following these results, Thumarat et al. [210] tested the best performing variant, A68V/T253P double mutant, with 3PET and PET film as substrates in the presence of $\mathrm{Ca}^{2+}$ at $\mathrm{pH} 7.0$ and $50^{\circ} \mathrm{C}$ for $3 \mathrm{~h}$. After $4 \mathrm{~min}$ of incubation, 3PET was depolymerized to TPA and EG almost to completion, confirming TaEst1 PET depolymerization activity. In the assays with PET film, even though no weight-loss was recorded, evidence of hydrolyzation of ester bonds in the film surface with release of hydroxyl and carboxyl groups was observed. Less efficiency in PET film degradation compared with assays with substrate 3PET is likely due to the higher crystallinity of PET, and less availability for enzymatic degradation. Further efforts to increase TaEst1 $T_{m}$ to higher value than the $T_{g}$ of PET are essential to enhance enzymatic activity and applicability in large-scale PET depolymerization.

\subsection{Thermomyces lanuginosus Lipase (TlLip)}

\subsubsection{Discovery}

Thermomyces lanuginosus lipase ( $T l$ Lip, aka $T l L$ ) is produced by a basophilic fungus previously known as Humicola lanuginosa discovered by Tsiklinskaya et al. [212] in 1889. TlLip consist of a single chain of 269 amino acids, with a molecular weight of $31 \mathrm{kDa}$ and an isoelectric point of 4.4, being firstly purified in 1972 by Arima et al. [213]. The enzyme was applied in various industrial areas [214-217], including in the hydrolysis of PET, firstly reported by Brueckner et al. [218] in 2008.

\subsubsection{Structure}

The enzyme adopts the $\alpha / \beta$-hydrolase fold with a central $\beta$-sheet containing eight predominantly parallel $\beta$-strands, and five $\alpha$-helices. The catalytic triad is made up of Ser 146 , His258, and Asp201 and the oxyanion hole by Ser83 and Leu147 [219,220]. Three disulfide bonds (Cys22-Cys268, Cys36-Cys41, and Cys104-Cys107) have also been reported.

The residue Glu87 was suggested to take part in the electrostatic stabilization of the lipase active open-lid conformation and Trp89 was proposed to play an important role in hydrolytic reactions by interacting with the substrate [221-223].

Derewenda et al. [220] determined the first crystallographic structure of TlLip in its free state with a resolution of $1.84 \AA$ (PDB: 1TIB), in 1993. Presently, 25 crystallographic structures have been deposited in the Protein Data Bank. All structures are summarized and characterized in Table 10. 
Table 10. Crystallographic structures of TlLip.

\begin{tabular}{|c|c|c|c|c|c|}
\hline PDB Code & Resolution (Å) & Ligand & Mutation & Year of Deposition & Ref. \\
\hline 1TIB & 1.84 & Free & - & 1993 & [220] \\
\hline 1DT3 & 2.6 & Free & - & 2000 & [224] \\
\hline 1DT5 & 2.4 & Free & - & 2000 & [224] \\
\hline 1DTE & 2.35 & Free & - & 2000 & [224] \\
\hline 1DU4 & 2.5 & Free & - & 2000 & {$[224]$} \\
\hline $1 \mathrm{EIN}$ & 3 & didodecyl phosphatidylcholine & - & 2000 & [224] \\
\hline 1GT6 & 2.2 & Oleic acid & S146A & 2002 & [225] \\
\hline $4 \mathrm{GYH}$ & 2 & Free & - & 2012 & $\mathrm{~N} / \mathrm{A}$ \\
\hline 4EA6 & 2.3 & Free & - & 2012 & $\mathrm{~N} / \mathrm{A}$ \\
\hline $4 \mathrm{FLF}$ & 2.15 & TPP & - & 2012 & [226] \\
\hline 4GBG & 2.9 & Free & - & 2012 & $\mathrm{~N} / \mathrm{A}$ \\
\hline 4GHW & 2.6 & decanoic acid & - & 2012 & $\mathrm{~N} / \mathrm{A}$ \\
\hline 4GI1 & 2.43 & 16-hydroxypalmitic acid & - & 2012 & $\mathrm{~N} / \mathrm{A}$ \\
\hline 4GLB & 2.69 & p-nitrobenzaldehyde & - & 2012 & $\mathrm{~N} / \mathrm{A}$ \\
\hline 4GWL & 2.55 & Free & - & 2012 & $\mathrm{~N} / \mathrm{A}$ \\
\hline $4 \mathrm{KJX}$ & 2.1 & p-nitrobenzaldehyde and lauric acid & - & 2013 & $\mathrm{~N} / \mathrm{A}$ \\
\hline $4 \mathrm{~N} 8 \mathrm{~S}$ & 2.3 & p-nitrobenzaldehyde and ethyl acetoacetate & - & 2013 & $\mathrm{~N} / \mathrm{A}$ \\
\hline $4 \mathrm{~S} 0 \mathrm{X}$ & 2.1 & Lauric acid & - & 2015 & $\mathrm{~N} / \mathrm{A}$ \\
\hline 4ZGB & 2.3 & Free & - & 2015 & [226] \\
\hline $5 \mathrm{AP} 9$ & 1.8 & Free & $\mathrm{I} 186 \mathrm{C} / \mathrm{I} 255 \mathrm{C}$ & 2015 & [227] \\
\hline 6HW1 & 2.5 & Free & - & 2018 & [228] \\
\hline $6 \mathrm{OR} 3$ & 1.45 & Palmitic acid & - & 2020 & [229] \\
\hline $6 \mathrm{XOK}$ & 1.3 & $\begin{array}{l}\text { 2-hydroxy-3-(octadecanoyloxy)propyl } \\
\text { pentacosanoate }\end{array}$ & - & 2020 & [229] \\
\hline $6 \mathrm{XRV}$ & 1.43 & $\begin{array}{l}\text { 2-hydroxy-3-(octadecanoyloxy)propyl } \\
\text { pentacosanoate and caprylic acid }\end{array}$ & - & 2020 & [229] \\
\hline $6 \mathrm{XS} 3$ & 2.48 & $\begin{array}{l}\text { 2-hydroxy-3-(octadecanoyloxy)propyl } \\
\text { pentacosanoate and caprylic acid }\end{array}$ & - & 2020 & [229] \\
\hline
\end{tabular}

\subsubsection{Activity}

The optimal activity temperature and $\mathrm{pH}$ of $\mathrm{Tl}$ Lip were reported to be $45^{\circ} \mathrm{C}$ and 7 , respectively [230]. This enzyme was shown to be stable for $20 \mathrm{~h}$ at $60^{\circ} \mathrm{C}$ and $1 \mathrm{~h}$ at $65^{\circ} \mathrm{C}$, losing its activity at temperatures higher than $70^{\circ} \mathrm{C}$, being the melting temperature around 65 to $70{ }^{\circ} \mathrm{C}[227,230]$.

In-site mutagenesis in the amino acid Ser146 (S146A) resulted in an inactive form of the enzyme, confirming its role as part of the catalytic triad [231]. Replacing Glu87 with alanine (E87A) and Trp89 with phenylalanine and glutamate (W89F and W89E, respectively) resulted in lower specificity towards tributyrin, suggesting that these residues play a role in the substrate binding $[221,222]$. The mutation of Ile186 and Ile255 to cysteine was applied to introduce a new disulfide bond (I186C/I255C). The resulting disulfide bond decreased the thermostability by $5{ }^{\circ} \mathrm{C}$, meaning that the addition of a new disulfide bond destabilizes the secondary structure of TlLip [227]. These engineering efforts are summarized in Table S9.

TlLip was able to degrade both amorphous and semi-crystalline PET fibers (5 and $40 \%$ crystallinity, respectively) releasing about two times more TPA and MHET from amorphous fibers. However, the amount of product released is much too low when compared with Thermobifida fusca cutinase [218]. Interestingly, the enzyme released higher amounts of MHET than TPA, whereas the cutinase released similar amounts of both products. This shows that both enzymes have different reaction mechanisms [218]. 
The hydrolysis of the PET model substrate 3PET resulted in the release of TPA, BHET, MHET, BA, and HEB [185]. When in presence of the non-ionic detergent Triton X-100, conformational changes involving lid-opening of the lipase occur, resulting in a 7-fold increase of the overall degradation products [185]. When incubated solely with BHET, the lipase did not liberate significant amounts of TPA, showing that TlLip preference towards the other intermediate product MHET [185].

\subsubsection{Future Perspectives}

TlLip is a promising enzyme for application in PET degradation. However, the activity and thermostability of the wild type enzyme are too low for direct usage in the plasticdegrading industry. Determination of its structure complexed with PET substrate or its analogue could provide a better understanding of the key residues involved in the reaction and their conformation, yielding important clues for a rational understanding of its catalytic mechanism. Calculation of the kinetic parameters $\mathrm{k}_{\text {cat }}$ and $\mathrm{K}_{\mathrm{M}}$ for the wild type enzyme and different mutants, following in-site mutagenesis would also be of great importance for enhancing the activity and thermostability of this enzyme.

\subsection{Thermobifida fusca Carboxylesterase (TfCa)}

\subsubsection{Discovery}

TfCa is a highly hydrophobic carboxylesterase produced by Thermobifida fusca, a mesothermophilic bacterium known for its ability to degrade cellulose. This organism produces many biocatalysts, being a powerful resource for synthetic polymer degradation $[112,139,144]$. The enzyme's properties as a PET biocatalyst were firstly described by Oeser et al. [232] in 2010 .

\subsubsection{Structure}

TfCa is composed of 497 amino acids and exists as a monomer. Identical sequences with other carboxylesterases are also observed [233]. The catalytic triad includes amino acid residues Ser185, His415, and Glu319. The catalytic serine is inserted in a motif containing residues Glu184 and Ala186 [233]. There are currently no X-ray structures available for this enzyme, so no further structural information is known.

\subsubsection{Activity}

TfCa presents a low isoelectric point (4.8) and a thermostability between 30 and $50{ }^{\circ} \mathrm{C}$ [233]. When incubated with $p \mathrm{NPB}$ at temperatures above $55^{\circ} \mathrm{C}$, the enzyme completely loses its activity [234]. However, after immobilization of TfCa on SulfoLink resin (where an oligopeptide of glycine-serine-cysteine was added at the C-terminus), the enzyme exhibited an improvement of thermostability, maintaining $94 \%$ of its initial activity at $60{ }^{\circ} \mathrm{C}$ [234]. The carboxylesterase is capable of hydrolyzing PET nanoparticles at $50{ }^{\circ} \mathrm{C}$ and cyclic PET trimers at an optimum temperature of $60^{\circ} \mathrm{C}$ and $\mathrm{pH} 6$ [232,233]. The products released towards cyclic PET trimers are 1,2-ethylene-mono-terephthalate-mono(2hydroxyethyl terephthalate (EMT) (95\%), MHET (3\%), and BHET (2\%). The enzyme also showed a $\mathrm{K}_{\mathrm{M}}$ value of $0.5 \pm 9 \% \mathrm{mM}$ for cyclic PET trimers. Belisário-Ferrari et al. [235] analyzed the hydrolysis of 2PET and BHET by TfCa. The hydrolysis of the PET intermediate resulted in a 3.5-fold higher $\mathrm{k}_{\mathrm{cat}}\left(0.35 \mathrm{~min}^{-1}\right)$ and a 2-fold higher $\mathrm{K}_{\mathrm{M}}(0.085 \mathrm{~mL} / \mathrm{mg})$ when compared to 2PET (0.10 $\mathrm{min}^{-1}$ and $0.042 \mathrm{~mL} / \mathrm{mg}$ of $\mathrm{k}_{\text {cat }}$ and $\mathrm{K}_{\mathrm{M}}$, respectively). These results revealed a preferential hydrolysis of smaller esters by the carboxylesterase

Barth et al. [234] built a dual enzyme system with LCC and TfCa, employing the latter for the hydrolysis of MHET to TPA. In fact, the dual system resulted in a 2.4-fold increase of product release $(10.42 \pm 1.85 \mathrm{mM})$ when compared with that of TfCut2 $(4.44 \pm 0.57 \mathrm{mM})$. A significant increase of product release was also reported when the enzyme was coupled with TfCut2 [234]. Mutations on the sequence motif (E184Q, A186M, and E319D) of the carboxylesterase resulted in activity losses up to $88 \%$, confirming their important roles for the catalytic activity [233], as described in Table S10. 


\subsubsection{Future Perspectives}

As little information on $T f \mathrm{Ca}$ is currently available, determining the crystallographic structure of the enzyme would provide a deeper understanding of its activity at the molecular level. To reach the plastic-degrading industry standard levels, in-site mutagenesis should be applied for enhancement of thermostability and enzymatic activity.

\subsection{Lesser-Known Enzymes}

In addition to the 16 enzymes described in detail above, over the years, several other enzymes have been reported to exhibit some PET degrading ability. These are portrayed in this section.

\subsubsection{Enterobacter sp. HYI Esterase B (EsEstB)}

EsEstB, or EstB, is an esterase from Enterobacter sp. HYI isolated and characterized by Qiu et al. [236] in 2020. The enzyme was shown to be able to degrade BHET to MHET and TPA. The bacterial strain was isolated from a plastic waste treatment station and identified as an esterase after assays confirming esterase-like activity.

Qiu et al. [236] characterized EsEstB as sharing 99.7\% sequence similarity with esterase from Enterobacter hormaechei. However, no BHET or PET degrading activity has been reported for this enzyme. Sequence-based structure predictions revealed the typical $\alpha / \beta$ hydrolase fold, with a catalytic triad composed of residues Ser110, Asp158, and His190.

Activity assays showed maximum activity was attained at an optimal $\mathrm{pH}$ of 8.0 and temperature of $40{ }^{\circ} \mathrm{C}$. EsEstB degraded $80.8 \%$ of BHET in $120 \mathrm{~h}$ at a degradation rate of $6.73 \mathrm{mg} / \mathrm{L} \cdot \mathrm{h}$ to produce MHET and further degrades MHET to TPA. The ability for complete degradation of BHET, frequently used as the PET commercial model, is promising for future applications of EsEstB in PET degradation.

\subsubsection{HR29 Hydrolase (BhrPETase)}

BhrPETase, a hydrolase from thermophilic bacteria $H R 29$, was recently overexpressed in Bacillus subtilis by Xi et al. [237] and shown to fully degrade PET. HR29 is involved in the biochemical nitrogen cycle and was identified by Kato et al. [238] in a long-term cultivation of thermophiles. Xi et al. [237] expressed BhrPETase in B. subtilis and characterized it. The enzyme shares $94 \%$ sequence identity with LCC, differing in 16 amino acid residues only.

BhrPETase is a 275 amino acid residue enzyme with a conserved $\alpha / \beta$-hydrolase fold and one disulfide bridge (Cys275-Cys292). The catalytic triad is made up of residues Ser165, His242, and Asp210.

Given the advantages of expressing enzymes in B. subtilis due to this bacterium being non-pathogenic, robust, and highly studied, Xi et al. [237] expressed LCC, IsPETase, and BhrPETase to compare activity and thermal stability. Enzymatic activity was firstly characterized with a series of monoesters of varying lengths, and then specific PET degrading activity was measured using BHET and low crystallinity PET powder as substrates. Depolymerization of PET substrates yielded TPA as the major product, and BHET and MHET in lower amounts. Similar to other PET degrading enzymes, BhrPETase exhibited higher activity on shorter acyl-chain length substrates, and maximum activity at a $\mathrm{pH}$ of 6 to 8 . BhrPETase showed remarkable thermal stability by linearly increasing activity from 30 to $90{ }^{\circ} \mathrm{C}$, contrary to LCC that, although highly stable, suffered an activity decrease above $80^{\circ} \mathrm{C}$. Furthermore, BhrPETase $\mathrm{T}_{\mathrm{m}}$ reached $110^{\circ} \mathrm{C}$, the highest known to PET degrading enzymes, and increased by $6.4^{\circ} \mathrm{C}$ in the presence of $\mathrm{Ca}^{2+}$ ions. Comparison with LCC suggests Glu208, Asp238, and Ser283 as probable calcium binding residues. BhrPETase proved highly active on PET powder complete degradation to BHET, MHET, and TPA, with the highest conversion occurring at $70^{\circ} \mathrm{C}$.

Although there is no three-dimensional structure available for BhrPETase, the high similarity with LCC justified the design of an LCC-based homology model. The differences found in the sequence might explain the higher thermal stability of BhrPETase regarding 
LCC. Xi et al. [237] proposed that Ser175 and Gln202 (which occupy the positions of Ala175 and Val202 in LCC) establish hydrogen bonds with nearby residues, replacing the hydrophobic interactions in LCC. Furthermore, two proline residues in BhrPETase (Pro199 and Pro248) occupy the positions of a serine and an asparagine in a loop region in LCC. It has been argued [237] that these proline residues might result in higher rigidity of the loop contributing to thermal stability of the overall structure.

Overall, BhrPETase is a novel enzyme with high PET degrading activity, thermal stability, and a $T_{m}$ higher than the $T_{g}$ of PET, features that make it a very promising enzyme for further studies on the biodegradation of PET.

\subsubsection{Bacillus subtilis Lipase (BsEstB)}

BsEstB, a Bacillus subtilis lipase, was firstly isolated by Eggert et al. [239] in 2000. This enzyme was demonstrated to have PETase-like activity by Ribitsch et al. [53] in 2011. $B s$ EstB is an intracellular p-nitrobenzylesterase with a molecular weight of $55.2 \mathrm{kDa}$ and an isoelectric point of 4.9. It was identified as belonging to the $\alpha / \beta$-hydrolase family, similar to most PET hydrolytic enzymes, and the catalytic triad is made up of residues Ser189, His399, and Glu310. The enzyme shares a 99\% similarity with typical B. subtilis and carboxylesterase type B [53].

Activity for PET degradation was firstly identified in a large screening of 250 enzymes with 3PET on agar plates. Subsequently to identification, isolation and expression, BsEstB was submitted to activity assays with PET film and BHET [53]. The enzyme is able to degrade PET to completion, as the major products of hydrolysis are TPA and benzoic acid. MHET was identified as a minor product and a rapidly degraded intermediate. Maximum activity was registered at $40^{\circ} \mathrm{C}$ and $\mathrm{pH} 7$ [53] The ability to completely depolymerize PET to its constituent monomers while being an intracellular enzyme is uncommon, and suggests this lipase is an ideal target for further PET degrading assays and studies.

\subsubsection{Streptomyces scabies Sub1 (ScSub1)}

ScSub1, commonly known as Sub1, is a cutinase-like enzyme from Streptomyces scabies, a gram-positive plant pathogen bacterium [240]. The protein was first discovered per identification of the gene sub1, which showed specific expression in the presence of cutin and suberin [241], and was recently characterized and subjected to specific activity assays [240]. Sequence similarity to fungal cutinases led to the classification of ScSub1 as a cutinase-like enzyme.

The structure for ScSub1 [240] was predicted using the ESyPred3D [242] server with a cutinase from Aspergillus oryzae (PDB: 3GBS) as a template. The resulting structure suggests a canonical $\alpha / \beta$-cutinase-like fold, made up of a five parallel strands central $\beta$-sheet surrounded by ten $\alpha$-helices. The same prediction suggests the existence of a catalytic triad composed of Ser114, His195, and Asp182, and two disulfide bonds, Cys31-Cys103 and Cys178-Cys185. The total molecular weight of ScSub1 is estimated to be $25 \mathrm{kDa}$, which includes mature ScSub1 with $18.7 \mathrm{kDa}$ and a His-tag of $4.9 \mathrm{kDa}$.

PET degradation by ScSub1 was assessed by measuring the amount of TPA produced, at $\mathrm{pH} 7.5$ and $37{ }^{\circ} \mathrm{C}$ for 20 days. The substrate used was commercially available PET granules, and TPA production remained constant during the assay time, resulting in a proportional relationship between incubation time and TPA release. Furthermore, the stability of $S c S u b 1$ at $50{ }^{\circ} \mathrm{C}$ was evaluated, and even though $S c S u b 1$ kept active, TPA production diminished with incubation time, suggesting progressive loss of stability and catalytic activity.

Interestingly, ScSub1 showed esterase activity by successfully degrading different substrates such as $p$-nitrophenyl butyrate, $p$-nitrophenyl octanoate, $p$-nitrophenyl decanoate, and $p$-nitrophenyl dodecanoate. Activity of ScSub1 on cutin and suberin was also confirmed and showed to be stable at room temperature over a 20-day period. 


\subsubsection{Pseudomonas mendocina Cutinase (PmCut)}

PmCut, aka PmC, is a cutinase from Pseudomonas mendocina first described as a PET degrading enzyme by Kellis et al. [243] in a patent proposition for enzymatic modification of polyesters in 2002. Inspired by this finding, Ronkvist et al. [157] included PmCut in a comparative study with FsCut and HiCut towards the understanding of the effect of PET crystallinity percentage on activity. Using two PET samples as substrate, the authors concluded that $P m$ Cut had the highest affinity for low crystallinity PET, having resulted in $5 \%$ PET film weight loss after $96 \mathrm{~h}$ at $50{ }^{\circ} \mathrm{C}$. Degradation rate of $\mathrm{PmCut}$ was lower than that of HiCut but higher than FsCut, presumably due to differences in thermal stability and optimal activity temperature. From the available data, it is to be expected that $P m C$ ut has a high potential as a PET degrading cutinase. However, further biochemical, and structural studies are essential to confirm enzymatic structure, sequence, and specific role in PET degradation.

\subsubsection{PET1-PET13}

Danso et al. [244] identified 504 possible PET hydrolytic enzyme candidates through an automatic search algorithm. Of these, 13 enzymes were selected and named PET1 through PET13. Activity of PET2, 5, 6, and 12 was verified through assays on agar plates containing PET nanoparticles-all four enzymes showed activity.

Furthermore, PET2 and PET6 were subjected to additional activity assays and biochemical characterization. PET2 showed high thermal stability, since it maintained PET degrading activity up to $90^{\circ} \mathrm{C}$, which is highly promising given the amorphous state of PET polymer at this temperature [244].

The initial search across several databases revealed the most promising candidates, selected from similarities with experimentally proven PET degrading enzymes, are mainly from three phyla: Proteobacteria, Actinobacteria, and Bacteroidetes. Furthermore, most sequences with high similarity originate from samples containing crude oil, similar to how ScSub1 was first discovered [244].

Database query for novel PET degrading enzymes followed a set of criteria inspired by enzymes with well characterized activity on PET, namely the presence of a conserved Ser-His-Asp catalytic triad in the vicinity of an oxyanion hole made up of a Met residue and an aromatic residue, a terminal disulfide bridge essential for enzymatic thermostability, and a conserved $\operatorname{DxDxR}(\mathrm{Y}) \times x F(\mathrm{~L}) \mathrm{C}$ sequence near the first cysteine of the disulfide bridge. Even though no further characterization of these enzymes has been published, the experimentally validated metagenomic methodology search used and the most promising enzymes identified suggest an encouraging path for identifying novel enzymes and microorganisms with relevant PET degrading activity.

All 13 PET hydrolytic enzymes identified by Danso et al. [244] are described in the UniProt database-Table 11 summarizes the UniProt access codes and general sequence characterization for these enzymes. There are no three-dimensional structures available for these proteins, and therefore specific structural motifs cannot be characterized and described with certainty. However, given the privileged knowledge of the amino acid sequence of each enzyme, several in silico sequence-based structure prediction methods could be used to combat the lack of an experimentally solved structure and allow for a deeper understanding of the determined activity rates and thermostability.

From the 13 PET enzymes, PET2, 5, 6, and 12 were tested for PET degrading activity against agar plates also containing nanoparticles of PCL. PCL was used as a model substrate, as the hydrolase ability for this substrate correlates with activity on higher complexity PET substrates. All four enzymes exhibited activity and produced halos in the plates. Furthermore, PET2 retained $80 \%$ of relative activity at $90{ }^{\circ} \mathrm{C}$ after an incubation period of $5 \mathrm{~h}$ with a series of $p$ NP esters, meaning it is even more stable than LCC.

PET2 ability to remain active at higher drastic temperatures makes it an extremely attractive enzyme for PET degradation. When $100 \mu \mathrm{g}$ of enzyme were tested with $14 \mathrm{mg}$ amorphous PET as substrate, $900 \mu \mathrm{M}$ of TPA were produced after a $24 \mathrm{~h}$ period incubation, 
confirming PET2 demonstrates interesting PET degrading activity and is able to fully degrade it to TPA.

Table 11. Summary of information known of enzymes PET 1-13.

\begin{tabular}{cccc}
\hline Pet No. & UniProt Code & Sequence Length & Organism \\
\hline 1 & E8U721 & 315 & Deinococcus maricopensis \\
\hline 2 & C3RYL0 & 308 & uncultured bacterium \\
\hline 3 & A0A0F9X315 & 300 & marine sediment metagenome \\
\hline 4 & N6VY44 & 295 & Marinobacter nanhaiticus \\
\hline 5 & R4YKL9 & 310 & Oleispira antarctica \\
\hline 6 & A0A1Z2SIQ1 & 298 & Vibrio gazogenes \\
\hline 7 & Q8RR62 & 304 & Acidovorax delafieldii \\
\hline 8 & P19833 & 319 & Moraxella sp. \\
\hline 9 & A0A0D4L7E6 & 313 & Psychrobacter sp. \\
\hline 10 & UPI00064655D2 & 292 & Methylibium sp. \\
\hline 11 & UPI0003660256 & 292 & Caldimonas manganoxidans \\
\hline 12 & A0A0G3BI90 & 298 & Burkholderiales bacterium \\
\hline 13 & A0A1F4G492 & 283 &
\end{tabular}

\subsubsection{Thermomonospora curvata Cutinases 0390 and 1278 (TcCut0390 and TcCut1278)}

TcCut0390 and TcCut1278, aka Tcur0390 and Tcur1278, are two cutinases from Thermomonospora curvata sharing $82 \%$ sequence identity [245]. T. curvata is a facultative aerobic thermophilic bacterium first isolated from plant composts [246-248]. Bacterial optimal growth conditions are $\mathrm{pH}$ from 7.5 to 11 and a temperature of $50^{\circ} \mathrm{C}$, but weak growth has been registered up to $65^{\circ} \mathrm{C}$ [245].

Wei et al. [245] isolated and expressed these proteins and characterized their structure from an homology model. Further molecular docking and molecular dynamics studies allowed for binding site and structural stability characterization. Both TcCut0390 and TcCut1278 assume a canonical $\alpha / \beta$-hydrolase fold with a catalytic triad composed of residues Ser130, His207, Asp176. The catalytic residues and the substrate-binding groove are solvent-exposed and located in the surface of the proteins. TcCut1278 is more thermally stable than TcCut0390, and they both hydrolyze $p$ NPB and PET nanoparticles. Given the low thermal stability of both enzymes at temperatures higher than $55^{\circ} \mathrm{C}$, their applicability in PET degradation campaigns is highly conditioned and dependent on future engineering efforts to increase enzymatic catalytic turnover and melting temperatures.

The ability of TcCut0390 and TcCut1278 to hydrolase polyurethane (PU) has recently been characterized by Islam et al. [249] and Schmidt et al. [250].

\subsubsection{Thermobifida halotolerans Esterase (ThEst)}

ThEst, aka Thh_Est, is an esterase from Thermobifida halotolerans, which was characterized as a PET degrading enzyme by Ribitsh et al. [54]. This 262 amino acid enzyme is highly similar to TaEst119 and several cutinases from T. cellulosilytica. Sequence analysis led to the prediction of an $\alpha / \beta$-serine hydrolase fold, with residue Ser131 as the catalytic triad serine, inserted in the typical GxSxG motif. Polyester hydrolysis ability of ThEst was firstly tested with 3PET in a $2 \mathrm{~h}$ activity assay at $50{ }^{\circ} \mathrm{C}$ and $\mathrm{pH} 7.0$. Degradation of 3PET resulted in the release of benzoic acid (BA) and 2-hydroxyethyl benzoate (HEB) as the main products, and MHET and TPA in residual amounts, which suggests an exo-type hydrolysis mechanism. Consequently, degradation of PET film resulted in TPA and MHET release with no BHET detected, indicating ThEst was able to degrade PET to completion. 
Comparisons with Thermobifida cutinases revealed similar degradation potentials and rates, differing from the trend of esterases being less efficient PET hydrolases than cutinases.

\section{Microorganisms with PET Degradation Activity}

The evidence that a given organism can degrade PET or other polyester materials and use them as a carbon source frequently leads to the identification of a PET degrading enzyme. However, in many cases it is not possible (or it has not yet been done) to identify or isolate the specific enzyme responsible for PET depolymerization. In this section, some of these microorganisms are described.

Liebminger et al. [251] screened for microorganisms with PET degradation activity by incubating environmental samples with 3PET as the only carbon source for 10 days. From the active samples, a fungal strain identified as Penicillium citrinum showed the best growth. Although an enzyme was purified, characterization and identification of this presumed polyesterase was not possible. The experiments with PET fabric and 3PET as substrates revealed optimal conditions for enzymatic activity to be $30^{\circ} \mathrm{C}$ and $\mathrm{pH} 8.2$. The enzyme successfully altered the surface of the PET fabric and degraded 3PET to TPA, MHET, BHET, and BA. Given the described results, it is to be expected that further biochemical and structural characterization of this novel polyesterase may reveal a new promising PET degrading enzyme.

Costa et al. [252] showed Yarrowia lipolytica, a widely used yeast, had PET degrading activity. Y. lipolytica was cultured with amorphous PET, PET oligomer 3, post-consumer PET (with high crystallinity), and PET monomers TPA, monoethylene glycol (MEG), and BHET. Highest activity was registered for the amorphous PET samples, with MHET as the main product and TPA as a residual released product. This suggests MHET has an inhibitory effect on the yeast, hindering complete degradation of PET to TPA. Although the yeast was able to use the monomers as a carbon source in the absence of other substrates, the accumulation of PET degradation products seemed to be toxic for yeast growth, and consumption rate was higher for PET oligomers than for the monomers. The described results show that Yarrowia lipolytica is able to express PET degrading enzymes, as evidenced by the release of MHET in large quantities, and further isolation and expression of these enzymes is of high importance for future PET degrading yeast studies.

Arguing that using individual purified enzymes to break down PET might not be the most promising strategy, Roberts et al. [253] showed that a bacterial consortia containing Bacillus and Pseudomonas species able to use PET as a sole carbon source resulted in more effective PET degradation and surface modification activity. The consortia were identified in a screening of soil samples for lipase activity, and several consortia were built and further analyzed after initial lipase activity was confirmed. PET hydrolytic activity was demonstrated by the depolymerization of amorphous PET to BHET, TPA, and EG by the bacterial consortia. Individual strains identified within the consortia were tested as well, but activity and product release were always slower and lower than in the assays with the full consortia. It was therefore concluded that the bacterial strains in the consortia acted on PET in a synergistic manner, and complete degradation was only possible in the presence of all bacterial strains and respective secreted enzymes. Identification of individual secreted enzymes was not possible, which the authors attributed to biofilm formation within the consortia, and natural tendency of the secreted enzymes to bind the PET sample, hindering isolation.

\section{Potential PET Hydrolases with Activity to Be Confirmed}

AoCut, a cutinase from Aspergillus oryzae, has been shown to degrade PCL and theorized to have a similar effect on PET $[254,255]$. The two three-dimensional structures available for AoCut (PDB: 3GBS and 3QPD) reveal an $\alpha / \beta$-serine hydrolase fold, with a central $\beta$ sheet flanked by $\alpha$ helixes on either side. The catalytic triad (Ser126, Asp181, His194) and oxyanion hole (Ser48 and Gln127) are similar to the reported residues in typical enzymes with PET degrading activity. The two major structural differences that 
differentiate $\mathrm{AoCut}$ are FsCut the additional disulfide bridge (Cys63-Cys76, in addition to the two well conserved Cys37-Cys115 and Cys177-Cys184) and a longer and deeper groove near the active site. These features could explain the higher thermal stability of AoCut, and also higher activity on PCL [255]. Efforts by Shirke et al. [254] to increase thermal stability via rational mutation design have been successful in increasing $\mathrm{T}_{\mathrm{m}}$ by $6{ }^{\circ} \mathrm{C}$, but with a negative effect on PCL degrading activity. It is widely suggested that AoCut will have a hydrolytic effect on PET polymer, but only specific experimental activity assays can confirm this assumption.

Almeida et al. [256] built a reference data set with 15 highly studied PET degrading enzymes to scan a 52 genome sequence dataset of the Streptomyces genus. An esterase from Streptomyces sp., SM14est, was identified as having the highest potential as PETase-like enzyme, and was isolated, expressed and subjected to activity assays with PCL. Having degraded PCL, and given the presence of PETase-like characteristics, it is suggested that this enzyme is a potential PET hydrolase. A homology model revealed a structure similar to most PET degrading cutinases, with nine $\beta$-sheets and seven $\alpha$-helixes. The catalytic triad was identified as Ser156, Asp202, His234; however, no disulfide bridge was predicted for the enzyme. Comparisons with IsPETase structures revealed a similar binding subsite I, but different binding subsite II. Molecular docking calculations predicted a binding mode for BHET like the one observed in IsPETase, supporting the authors' thesis that SM14est is likely a PET degrading enzyme. However, besides the obvious need for experimental confirmation, the absence of any disulfide bond is a never-seen feature in PET hydrolases, and might greatly hinder thermal stability and, consequently, activity.

Huang et al. [257] reported an esterase from Thermobifida fusca named Tf AXE, with high similarity with $\mathrm{T} f \mathrm{HCut}$ and other highly active PET degrading enzymes. Even though specific activity against PET or other polyester polymers has not yet been described for this enzyme, it is consensually regarded as a likely PETase-like enzyme and considered of major interest in state-of-the-art PET bioremediation studies.

\subsection{Thermobifida alba Esterase 119 (TaEst119)}

This enzyme is closely related with TaEst1, a confirmed PET hydrolase, and is therefore a highly promising PET degrading enzyme.

\subsubsection{Discovery}

TaEst119 is an esterase from Thermobifida alba, a mesophilic bacterium [247]. The strain AHK119 possesses two tandem genes codifying two highly similar enzymes-TaEst119 and TaEst 1 . These enzymes share $95 \%$ identity and $98 \%$ similarity $[45,210]$.

TaEst119 has a molecular weight of $30 \mathrm{kDa}, 300$ amino acids, and was firstly identified in 2010 by Hu et al. [258] that described its potential as a PETase-like enzyme, even though no specific assays with PET related substrates were reported. However, Thumarat et al. [57] confirmed TaEst119 activity on agar plates containing the polyesters PCL, PBSA, PBS, PDLA, PLLA, and PHB. The molecular docking of PET with a TaEst119 three-dimensional structure was also reported by Thuramat, showing a good fitting of the docked PET unit in the active site for optimal catalyses.

\subsubsection{Structure}

In 2012, Thuramat et al. [57] predicted the TaEst119 structure from its sequence by modeling a 3D structure based on the well characterized homologous lipase, S. exfoliatus M11. The group reported that the enzyme displays an $\alpha / \beta$-hydrolase fold with central nine stranded parallel $\beta$-sheets flanked by seven $\alpha$-helices on both sides. Existent crystallographic structures for TaEst119 are summarized in Table 12. Later in the same year, the first crystallographic structure was reported by Kitadokoro et al. [57] with a resolution of $1.76 \AA$. The structure confirmed that this enzyme exists as a monomer and exhibits an $\alpha / \beta$-hydrolase fold with a central twisted $\beta$-sheet of nine $\beta$-strands, flanked by nine $\alpha$-helices on both sides. The catalytic triad, which is located in the loops between the 
$\beta$-sheets and helices, is composed of Ser169, His247, and Asp215. The oxyanion hole is made up of amino acid residues Met170 and Tyr99. However, in a later report by Thumarat et al. [210], Met166 and Tyr99 are suggested as the residues comprising the oxyanion hole. A disulfide bond (Cys280-Cys298) is displayed in the C-terminal region.

Table 12. Crystallographic structures of TaEst119.

\begin{tabular}{cccccc}
\hline PDB Code & Resolution $(\AA)$ & Ligand & Mutation & Year of Deposition & Ref. \\
\hline 3VIS & 1.76 & - & - & 2011 & {$[259]$} \\
\hline 3WYN & 1.68 & - & - & 2014 & {$[45]$} \\
\hline 6AID & 1.3 & Ethyl lactate & - & 2018 & {$[260]$} \\
\hline
\end{tabular}

A crystallographic structure was also developed by Kawai et al. [45] with a resolution of $1.68 \AA$. Two $\mathrm{Ca}^{2+}$ binding sites were shown to occupy a position in the loop regions $\alpha 7-$ $\beta 9$ and $\beta 9-C$-terminal. Lastly, Kitadokoro et al. [260] determined the structure complexed with ethyl lactate (EL) with a resolution of $1.3 \AA$, in 2018 . Here, the group confirmed the existence of the previously reported $\mathrm{Ca}^{2+}$ binding sites, also reporting two other $\mathrm{Ca}^{2+}$ binding sites, amounting to a total of $4 \mathrm{Ca}^{2+}$ binding sites. Studies on the active site revealed it is formed by a long narrow groove. The terminal side of the groove is comprised of Tyr99, Met170, Trp194, and Ile217, which involve lactate (LAC). Tyr99, Ile217, and Phe248 also form hydrophobic interactions with EL.

\subsubsection{Activity}

The optimal conditions for TaEst119 activity have been reported to be around 45 to $55{ }^{\circ} \mathrm{C}$ and $\mathrm{pH} 6.0$, where the enzyme loses $50 \%$ of its activity at $60{ }^{\circ} \mathrm{C}$ [258].

Among the $p$-nitrophenyl acyl esters, TaEst119 revealed higher enzymatic activity towards $p$ NPB substrate, resulting in a $\mathrm{k}_{\text {cat }}$ of $4.48 \pm 0.21 \mathrm{~s}^{-1}$ [57], and a specific activity of $2.30 \pm 0.02 \mathrm{U} / \mathrm{mg}$ in the absence of $\mathrm{Ca}^{2+}$ and $8.29 \pm 0.03 \mathrm{U} / \mathrm{mg}$ in the presence of $300 \mathrm{mg}$ of $\mathrm{Ca}^{2+}[210]$.

The substitution of the N-terminus residues correlated to higher enzymatic activity towards $p$ NPB [57]. Replacing Ala68 with isoleucine, valine, or leucine results in an increase in hydrophobicity; however, the opposite is observed when the residue is replaced by tyrosine, tryptophan, or proline. The variant A68V resulted in the highest activity. The thermostability was significantly increased when Ser219, located in the substratedocking loop, was replaced with proline (S219P). The double mutant (A68V/S219) resulted in a specific activity of $115 \pm 3.5$ and $299 \pm 10.1 \mathrm{U} / \mathrm{mg}$ in the absence and presence of $\mathrm{Ca}^{2+}$, respectively [210]. In the presence of $\mathrm{Ca}^{2+}$ the enzymatic activity and thermostability were remarkably enhanced, suggesting that even though the secondary structure is not affected by $\mathrm{Ca}^{2+}$, the cations stabilize the tertiary protein structure $[45,57,210,260]$.

\subsubsection{Proposed Mechanism}

No catalytic mechanism towards PET degradation has been reported. However, Kitadokoro et al. [260] proposed the mechanism for ethyl lactate, which is the analogue of another plastic polymer, polylactic acid (PLA).

According to their proposal, His247 deprotonates Ser169, which becomes a nucleophile able to attack the carbonyl carbon atom of EL, resulting in the movement of a pair of electrons from the double bond of the carbonyl oxygen towards the oxygen, which are stabilized by the main-chain nitrogen atoms comprising the oxyanion hole (Tyr99 and Met170). This process results in the formation of a tetrahedral intermediate. The covalent electrons of the ester bond between the oxygen and the carbon in EL attack the hydrogen of Hys247, breaking the ester bond. The electrons that previously moved from the carbonyl oxygen double bond restore the double bond, leading to the collapse of the tetrahedral intermediate, which forms an acyl-enzyme intermediate and ethanol. The same pair of electrons move again to the oxygen and a water molecule bonds to the carbon atom 
of the acyl-enzyme. The hydrolytic attack by the water molecule towards the nitrogen of His247 leads to another intermediate and the release of ethanol. Lastly, the proton originated from the hydrolytic attack is transferred to Ser169, regenerating the catalytic triad, and thus releasing lactate.

\subsubsection{Future Perspectives}

To implement TaEst119 in the plastic-degrading industry for catalyses of PET substrates, enzymatic activity and thermostability need to be enhanced. For a better understanding of the enzymatic activity, studies of TaEst119 complexed with PET substrates should be developed.

\section{Summary and Outlook}

As awareness increases about how plastics affect human health and the environment, so do the efforts by the scientific community in finding solutions. The evolution of bioengineering and bioremediation technology and research has allowed for the progressive discovery of several enzymes with plastic degradation ability, mainly for PET, as one of the most produced, used, and discarded plastics.

Although several of these enzymes are highly promising, their activities and thermal stabilities are still far from the desirable thresholds for efficient and large-scale applicability. A summary of the best engineering efforts for the described enzymes is presented in Table 13. Many of the enzymes with highly characterized structures are yet to be the target of mutagenic studies, and their potential activities remain to be explored. More studies are required to unharness the full potential of these enzymes for PET degradation.

Table 13. Mutations corresponding to the highest activity and best performance for several of the main PET degrading enzymes described.

\begin{tabular}{ll}
\hline \multicolumn{1}{c}{ Enzyme } & \multicolumn{1}{c}{ Best Performing Mutation } \\
\hline IsPETase & S214H/I168R/W159H/S188Q/R280A/A180I/G165A/Q119Y/L117F/T140D \\
\hline IsMHETase & W397A \\
\hline PaPETase & Y250S \\
\hline \multirow{2}{*}{ LCC } & F243I/D238C/S283D/Y127G \\
\cline { 2 - 2 } & F243I/D238C/S283C/T96M \\
\cline { 2 - 2 } TfHCut & F243W/D238C/S283C/Y127G \\
\hline SvCut190 & Q132A/T101A \\
\hline TfCut2 & D204C/E253C/D174R \\
\hline TcCut2 & R29N/A30V \\
\hline & L182A \\
\cline { 2 - 2 } FsCut & L81A \\
\cline { 2 - 2 } TaEst1 & V184A \\
\hline
\end{tabular}

Supplementary Materials: The following are available online at https:/ /www.mdpi.com/article/10 .3390/ijms222011257/s1, Table S1: Mutagenesis assays with IsPETase, Table S2: Mutagenesis assays with IsMHETase, Table S3: Mutagenesis assays with PaMHETase, Table S4: Mutagenesis assays with LCC, Table S5: Mutagenesis assays with TfHCut, Table S6: Mutagenesis assays with TcCut, Table S7: Mutagenesis assays with TlLip, Table S8: Mutagenesis assays with SvCut190, Table S9: Mutagenesis assays with TfCa, Table S10: Mutagenesis assays with TaEst1. 
Author Contributions: Conceptualization, S.F.S.; investigation, R.P.M., J.M.C. and S.F.S.; data curation, R.P.M., J.M.C. and S.F.S.; writing—original draft preparation, R.P.M. and J.M.C.; writing-review and editing, S.F.S.; supervision, S.F.S.; project administration, S.F.S.; funding acquisition, S.F.S. All authors have read and agreed to the published version of the manuscript.

Funding: This research was supported by national funds from the Portuguese Foundation for Science and Technology (FCT) under the scope of the strategic funding UIDP/04378/2020 and UIDB/04378/2020. R.P.M. acknowledges the PhD fellowship 2020.09087.BD funded by FCT. S.F.S. acknowledges FCT by funding 2020.01423.CEECIND/CP1596/CT0003.

Conflicts of Interest: The authors declare no conflict of interest.

\section{References}

1. Amobonye, A.; Bhagwat, P.; Singh, S.; Pillai, S. Plastic Biodegradation: Frontline Microbes and Their Enzymes. Sci. Total Environ. 2021, 759, 143536. [CrossRef] [PubMed]

2. Geyer, R.; Jambeck, J.R.; Law, K.L. Production, Use, and Fate of All Plastics Ever Made. Sci. Adv. 2017, 3, e1700782. [CrossRef] [PubMed]

3. Samak, N.A.; Jia, Y.; Sharshar, M.M.; Mu, T.; Yang, M.; Peh, S.; Xing, J. Recent Advances in Biocatalysts Engineering for Polyethylene Terephthalate Plastic Waste Green Recycling. Environ. Int. 2020, 145, 106144. [CrossRef]

4. Gibb, B.C. Plastics Are Forever. Nat. Chem. 2019, 11, 394-395. [CrossRef] [PubMed]

5. Feil, A.; Pretz, T. Chapter 11-Mechanical Recycling of Packaging Waste; Letcher, T.M., Ed.; Academic Press: Cambridge, MA, USA, 2020; pp. 283-319.

6. Plastics-The Facts 2020. Available online: https://www.plasticseurope.org/en/resources/publications/4312-plastics-facts-2020 (accessed on 21 April 2021).

7. Chemistry Can Help Make Plastics Sustainable-but It Isn't the Whole Solution. Nature 2021, 590, 363-364. [CrossRef] [PubMed]

8. Nielsen, T.D.; Hasselbalch, J.; Holmberg, K.; Stripple, J. Politics and the Plastic Crisis: A Review throughout the Plastic Life Cycle. WIREs Energy Environ. 2020, 9, e360. [CrossRef]

9. Wei, R.; Zimmermann, W. Biocatalysis as a Green Route for Recycling the Recalcitrant Plastic Polyethylene Terephthalate. Microb. Biotechnol. 2017, 10, 1302-1307. [CrossRef]

10. Lahive, E.; Walton, A.; Horton, A.A.; Spurgeon, D.J.; Svendsen, C. Microplastic Particles Reduce Reproduction in the Terrestrial Worm Enchytraeus Crypticus in a Soil Exposure. Environ. Pollut. 2019, 255, 113174. [CrossRef]

11. Selonen, S.; Dolar, A.; Jemec Kokalj, A.; Skalar, T.; Parramon Dolcet, L.; Hurley, R.; van Gestel, C.A.M. Exploring the Impacts of Plastics in Soil-The Effects of Polyester Textile Fibers on Soil Invertebrates. Sci. Total Environ. 2020, 700, 134451. [CrossRef]

12. Jung, J.-W.; Kang, J.-S.; Choi, J.; Park, J.-W. Chronic Toxicity of Endocrine Disrupting Chemicals Used in Plastic Products in Korean Resident Species: Implications for Aquatic Ecological Risk Assessment. Ecotoxicol. Environ. Saf. 2020, 192, 110309. [CrossRef]

13. Hopewell, J.; Dvorak, R.; Kosior, E. Plastics Recycling: Challenges and Opportunities. Philos. Trans. R. Soc. Lond. B Biol. Sci. 2009, 364, 2115-2126. [CrossRef] [PubMed]

14. Verma, R.; Vinoda, K.S.; Papireddy, M.; Gowda, A.N.S. Toxic Pollutants from Plastic Waste-A Review. Procedia Environ. Sci. 2016, 35, 701-708. [CrossRef]

15. Alabi, O.A.; Ologbonjaye, K.I.; Awosolu, O.; Alalade, O.E. Public and Environmental Health Effects of Plastic Wastes Disposal: A Review. J. Toxicol. Risk Assess. 2019, 5, 1-13.

16. Grigore, M.E. Methods of Recycling, Properties and Applications of Recycled Thermoplastic Polymers. Recycling 2017, 2, 24. [CrossRef]

17. Al-Salem, S.M.; Lettieri, P.; Baeyens, J. Recycling and Recovery Routes of Plastic Solid Waste (PSW): A Review. Waste Manag. 2009, 29, 2625-2643. [CrossRef]

18. Ragaert, K.; Delva, L.; Van Geem, K. Mechanical and Chemical Recycling of Solid Plastic Waste. Waste Manag. 2017, 69, 24-58. [CrossRef]

19. Scalenghe, R. Resource or Waste? A Perspective of Plastics Degradation in Soil with a Focus on End-of-Life Options. Heliyon 2018, 4, e00941. [CrossRef] [PubMed]

20. Schyns, Z.O.G.; Shaver, M.P. Mechanical Recycling of Packaging Plastics: A Review. Macromol. Rapid Commun. 2021, $42,2000415$. [CrossRef]

21. Al-Sabagh, A.M.; Yehia, F.Z.; Eshaq, G.; Rabie, A.M.; ElMetwally, A.E. Greener Routes for Recycling of Polyethylene Terephthalate. Egypt. J. Pet. 2016, 25, 53-64. [CrossRef]

22. Garcia, J.M.; Robertson, M.L. The Future of Plastics Recycling. Science 2017, 358, 870-872. [CrossRef] [PubMed]

23. Karayannidis, G.P.; Achilias, D.S. Chemical Recycling of Poly(Ethylene Terephthalate). Macromol. Mater. Eng. 2007, 292, 128-146. [CrossRef]

24. Paszun, D.; Spychaj, T. Chemical Recycling of Poly(Ethylene Terephthalate). Ind. Eng. Chem. Res. 1997, 36, 1373-1383. [CrossRef]

25. Focht, D.D. Biodegradation. Access Sci. 2021. [CrossRef]

26. Roohi; Bano, K.; Kuddus, M.; Zaheer, M.R.; Zia, Q.; Khan, M.F.; Ashraf, G.M.; Gupta, A.; Aliev, G. Microbial Enzymatic Degradation of Biodegradable Plastics. Curr. Pharm. Biotechnol. 2017, 18, 429-440. [CrossRef] [PubMed] 
27. Bryant, J.A.; Clemente, T.M.; Viviani, D.A.; Fong, A.A.; Thomas, K.A.; Kemp, P.; Karl, D.M.; White, A.E.; DeLong, E.F. Diversity and Activity of Communities Inhabiting Plastic Debris in the North Pacific Gyre. mSystems 2016, 1, e00024-16. [CrossRef] [PubMed]

28. Rose, R.-S.; Richardson, K.H.; Latvanen, E.J.; Hanson, C.A.; Resmini, M.; Sanders, I.A. Microbial Degradation of Plastic in Aqueous Solutions Demonstrated by CO(2) Evolution and Quantification. Int. J. Mol. Sci. 2020, 21, 1176. [CrossRef]

29. Montazer, Z.; Habibi Najafi, M.B.; Levin, D.B. Challenges with Verifying Microbial Degradation of Polyethylene. Polymers 2020, 12, 123. [CrossRef]

30. Kaushal, J.; Khatri, M.; Arya, S.K. Recent Insight into Enzymatic Degradation of Plastics Prevalent in the Environment: A Mini-Review. Clean. Eng. Technol. 2021, 2, 100083. [CrossRef]

31. Sousa, S.F.; Ramos, M.J.; Lim, C.; Fernandes, P.A. Relationship between Enzyme/Substrate Properties and Enzyme Efficiency in Hydrolases. ACS Catal. 2015, 5, 5877-5887. [CrossRef]

32. Vedrtnam, A.; Kumar, S.; Chaturvedi, S. Experimental Study on Mechanical Behavior, Biodegradability, and Resistance to Natural Weathering and Ultraviolet Radiation of Wood-Plastic Composites. Compos. Part B Eng. 2019, 176, 107282. [CrossRef]

33. Wei, R.; Tiso, T.; Bertling, J.; O'Connor, K.; Blank, L.M.; Bornscheuer, U.T. Possibilities and Limitations of Biotechnological Plastic Degradation and Recycling. Nat. Catal. 2020, 3, 867-871. [CrossRef]

34. Liu, C.; Shi, C.; Zhu, S.; Wei, R.; Yin, C.-C. Structural and Functional Characterization of Polyethylene Terephthalate Hydrolase from Ideonella Sakaiensis. Biochem. Biophys. Res. Commun. 2019, 508, 289-294. [CrossRef] [PubMed]

35. Carr, C.M.; Clarke, D.J.; Dobson, A.D.W. Microbial Polyethylene Terephthalate Hydrolases: Current and Future Perspectives. Front. Microbiol. 2020, 11, 2825. [CrossRef] [PubMed]

36. Webb, H.K.; Arnott, J.; Crawford, R.J.; Ivanova, E.P. Plastic Degradation and Its Environmental Implications with Special Reference to Poly(Ethylene Terephthalate). Polymers 2013, 5, 1-18. [CrossRef]

37. Nguyen, H.T.H.; Qi, P.; Rostagno, M.; Feteha, A.; Miller, S.A. The Quest for High Glass Transition Temperature Bioplastics. J. Mater. Chem. A 2018, 6, 9298-9331. [CrossRef]

38. Puspitasari, N.; Tsai, S.-L.; Lee, C.-K. Class I Hydrophobins Pretreatment Stimulates PETase for Monomers Recycling of Waste PETs. Int. J. Biol. Macromol. 2021, 176, 157-164. [CrossRef] [PubMed]

39. Chamas, A.; Moon, H.; Zheng, J.; Qiu, Y.; Tabassum, T.; Jang, J.H.; Abu-Omar, M.; Scott, S.L.; Suh, S. Degradation Rates of Plastics in the Environment. ACS Sustain. Chem. Eng. 2020, 8, 3494-3511. [CrossRef]

40. Frounchi, M. Studies on Degradation of PET in Mechanical Recycling. Macromol. Symp. 1999, 144, 465-469. [CrossRef]

41. Crippa, M.; Morico, B. Chapter 12-PET Depolymerization: A Novel Process for Plastic Waste Chemical Recycling. In Catalysis, Green Chemistry and Sustainable Energy; Basile, A., Centi, G., de Falco, M., Iaquaniello, G., Eds.; Elsevier Press: Amsterdam, The Netherlands, 2020; Volume 179, pp. 215-229.

42. Bartolome, L.; Imran, M.; Cho, B.; Al-Masry, W.; Kim, D. Recent Developments in the Chemical Recycling of PET. In Material Recycling-Trends and Perspectives; InTech: Rijeka, Croatia, 2012.

43. Kim, H.T.; Kim, J.K.; Cha, H.G.; Kang, M.J.; Lee, H.S.; Khang, T.U.; Yun, E.J.; Lee, D.-H.; Song, B.K.; Park, S.J.; et al. Biological Valorization of Poly(Ethylene Terephthalate) Monomers for Upcycling Waste PET. ACS Sustain. Chem. Eng. 2019, 7, 19396-19406. [CrossRef]

44. Bano, N.; Younas, T.; Shoaib, F.; Rashid, D.; Jaffri, N. Plastic: Reduce, Recycle, and Environment. In Environmentally-Benign Energy Solutions; Springer: Cham, Switzerland, 2020; pp. 191-208.

45. Kawai, F.; Thumarat, U.; Kitadokoro, K.; Waku, T.; Tada, T.; Tanaka, N.; Kawabata, T. Comparison of Polyester-Degrading Cutinases from Genus Thermobifida. In Green Polymer Chemistry: Biocatalysis and Materials II; ACS Symposium Series; American Chemical Society Press: Washington, DC, USA, 2013; Volume 1144, pp. 111-120.

46. Jog, J.P. Crystallization of Polyethyleneterephthalate. J. Macromol. Sci. Part C 1995, 35, 531-553. [CrossRef]

47. Biron, M. Mechanical Properties. In Material Selection for Thermoplastic Parts; William Andrew Publishing: Oxford, UK, 2016; pp. 261-337.

48. Schirrer, R. Damage Mechanisms in Amorphous Glassy PolymersCrazing. In Handbook of Materials Behavior Models; Academic Press: Cambridge, MA, USA, 2001; pp. 488-499.

49. Wei, R.; Breite, D.; Song, C.; Gräsing, D.; Ploss, T.; Hille, P.; Schwerdtfeger, R.; Matysik, J.; Schulze, A.; Zimmermann, W. Biocatalytic Degradation Efficiency of Postconsumer Polyethylene Terephthalate Packaging Determined by Their Polymer Microstructures. Adv. Sci. 2019, 6, 1900491. [CrossRef]

50. Danso, D.; Chow, J.; Streit, W.R. Plastics: Environmental and Biotechnological Perspectives on Microbial Degradation. Appl. Environ. Microbiol. 2019, 85, e01095-19. [CrossRef]

51. Müller, R.-J.; Schrader, H.; Profe, J.; Dresler, K.; Deckwer, W.-D. Enzymatic Degradation of Poly(Ethylene Terephthalate): Rapid Hydrolyse Using a Hydrolase from T. Fusca. Macromol. Rapid Commun. 2005, 26, 1400-1405. [CrossRef]

52. Yoshida, S.; Hiraga, K.; Takehana, T.; Taniguchi, I.; Yamaji, H.; Maeda, Y.; Toyohara, K.; Miyamoto, K.; Kimura, Y.; Oda, K. A Bacterium That Degrades and Assimilates Poly(Ethylene Terephthalate). Science 2016, 351, 1196-1199. [CrossRef]

53. Ribitsch, D.; Heumann, S.; Trotscha, E.; Herrero Acero, E.; Greimel, K.; Leber, R.; Birner-Gruenberger, R.; Deller, S.; Eiteljoerg, I.; Remler, P.; et al. Hydrolysis of Polyethyleneterephthalate by P-Nitrobenzylesterase from Bacillus Subtilis. Biotechnol. Prog. 2011, 27, 951-960. [CrossRef] 
54. Ribitsch, D.; Acero, E.H.; Greimel, K.; Dellacher, A.; Zitzenbacher, S.; Marold, A.; Rodriguez, R.D.; Steinkellner, G.; Gruber, K.; Schwab, H.; et al. A New Esterase from Thermobifida Halotolerans Hydrolyses Polyethylene Terephthalate (PET) and Polylactic Acid (PLA). Polymers 2012, 4, 617-629. [CrossRef]

55. Silva, C.; Da, S.; Silva, N.; Matamá, T.; Araújo, R.; Martins, M.; Chen, S.; Chen, J.; Wu, J.; Casal, M.; et al. Engineered Thermobifida Fusca Cutinase with Increased Activity on Polyester Substrates. Biotechnol. J. 2011, 6, 1230-1239. [CrossRef]

56. Kawai, F.; Oda, M.; Tamashiro, T.; Waku, T.; Tanaka, N.; Yamamoto, M.; Mizushima, H.; Miyakawa, T.; Tanokura, M. A Novel Ca2+-Activated, Thermostabilized Polyesterase Capable of Hydrolyzing Polyethylene Terephthalate from Saccharomonospora Viridis AHK190. Appl. Microbiol. Biotechnol. 2014, 98, 10053-10064. [CrossRef]

57. Thumarat, U.; Nakamura, R.; Kawabata, T.; Suzuki, H.; Kawai, F. Biochemical and Genetic Analysis of a Cutinase-Type Polyesterase from a Thermophilic Thermobifida Alba AHK119. Appl. Microbiol. Biotechnol. 2012, 95, 419-430. [CrossRef]

58. Sulaiman, S.; Yamato, S.; Kanaya, E.; Kim, J.-J.; Koga, Y.; Takano, K.; Kanaya, S. Isolation of a Novel Cutinase Homolog with Polyethylene Terephthalate-Degrading Activity from Leaf-Branch Compost by Using a Metagenomic Approach. Appl. Environ. Microbiol. 2012, 78, 1556-1562. [CrossRef]

59. Goldsmith, M.; Tawfik, D.S. Enzyme Engineering: Reaching the Maximal Catalytic Efficiency Peak. Curr. Opin. Struct. Biol. 2017, 47, 140-150. [CrossRef]

60. Sharma, A.; Gupta, G.; Ahmad, T.; Mansoor, S.; Kaur, B. Enzyme Engineering: Current Trends and Future Perspectives. Food Rev. Int. 2021, 37, 121-154. [CrossRef]

61. Hida, K.; Hanes, J.; Ostermeier, M. Directed Evolution for Drug and Nucleic Acid Delivery. Adv. Drug Deliv. Rev. 2007, 59, 1562-1578. [CrossRef]

62. Dubey, K.K.; Pramanik, A.; Ankush; Khushboo; Yadav, J. Chapter 12-Enzyme Engineering. In Biomass, Biofuels, Biochemicals; Singh, R.S., Singhania, R.R., Pandey, A., Larroche, C., Eds.; Elsevier: Amsterdam, The Netherlands, 2019 ; pp. 325-347.

63. Berman, H.M.; Westbrook, J.; Feng, Z.; Gilliland, G.; Bhat, T.N.; Weissig, H.; Shindyalov, I.N.; Bourne, P.E. The Protein Data Bank. Available online: rcsb.org (accessed on 22 September 2021).

64. Jumper, J.; Evans, R.; Pritzel, A.; Green, T.; Figurnov, M.; Ronneberger, O.; Tunyasuvunakool, K.; Bates, R.; Žídek, A.; Potapenko, A.; et al. Highly Accurate Protein Structure Prediction with AlphaFold. Nature 2021, 596, 583-589. [CrossRef]

65. Planas-Iglesias, J.; Marques, S.M.; Pinto, G.P.; Musil, M.; Stourac, J.; Damborsky, J.; Bednar, D. Computational Design of Enzymes for Biotechnological Applications. Biotechnol. Adv. 2021, 47, 107696. [CrossRef]

66. Labrou, N.E. Random Mutagenesis Methods for In Vitro Directed Enzyme Evolution. Curr. Protein Pept. Sci. 2010, 11, 91-100. [CrossRef] [PubMed]

67. Wolfenden, R. Benchmark Reaction Rates, the Stability of Biological Molecules in Water, and the Evolution of Catalytic Power in Enzymes. Annu. Rev. Biochem. 2011, 80, 645-667. [CrossRef]

68. Wolfenden, R. Degrees of Difficulty of Water-Consuming Reactions in the Absence of Enzymes. Chem. Rev. 2006, 106, 3379-3396. [CrossRef] [PubMed]

69. Ogata, H.; Goto, S.; Sato, K.; Fujibuchi, W.; Bono, H.; Kanehisa, M. KEGG: Kyoto Encyclopedia of Genes and Genomes. Nucleic Acids Res. 1999, 27, 29-34. [CrossRef]

70. Knudsen, M.; Wiuf, C. The CATH Database. Hum. Genom. 2010, 4, 207-212. [CrossRef]

71. Rajagopalan, S.; Wang, C.; Yu, K.; Kuzin, A.P.; Richter, F.; Lew, S.; Miklos, A.E.; Matthews, M.L.; Seetharaman, J.; Su, M.; et al. Design of Activated Serine-Containing Catalytic Triads with Atomic-Level Accuracy. Nat. Chem. Biol. 2014, 10, 386-391. [CrossRef]

72. Ordentlich, A.; Barak, D.; Kronman, C.; Ariel, N.; Segall, Y.; Velan, B.; Shafferman, A. Functional Characteristics of the Oxyanion Hole in Human Acetylcholinesterase. J. Biol. Chem. 1998, 273, 19509-19517. [CrossRef]

73. Daniel, R.M.; Danson, M.J. A New Understanding of How Temperature Affects the Catalytic Activity of Enzymes. Trends Biochem. Sci. 2010, 35, 584-591. [CrossRef]

74. Joo, S.; Cho, I.J.; Seo, H.; Son, H.F.; Sagong, H.-Y.; Shin, T.J.; Choi, S.Y.; Lee, S.Y.; Kim, K.-J. Structural Insight into Molecular Mechanism of Poly(Ethylene Terephthalate) Degradation. Nat. Commun. 2018, 9, 382. [CrossRef] [PubMed]

75. Austin, H.P.; Allen, M.D.; Donohoe, B.S.; Rorrer, N.A.; Kearns, F.L.; Silveira, R.L.; Pollard, B.C.; Dominick, G.; Duman, R.; El Omari, K.; et al. Characterization and Engineering of a Plastic-Degrading Aromatic Polyesterase. Proc. Natl. Acad. Sci. USA 2018, 115, E4350-E4357. [CrossRef] [PubMed]

76. Han, X.; Liu, W.; Huang, J.-W.; Ma, J.; Zheng, Y.; Ko, T.-P.; Xu, L.; Cheng, Y.-S.; Chen, C.-C.; Guo, R.-T. Structural Insight into Catalytic Mechanism of PET Hydrolase. Nat. Commun. 2017, 8, 2106. [CrossRef] [PubMed]

77. Weiland, M.H. Enzymatic Biodegradation by Exploring the Rational Protein Engineering of the Polyethylene Terephthalate Hydrolyzing Enzyme PETase from Ideonella Sakaiensis 201-F6. In Mechanistic Enzymology: Bridging Structure and Function; ACS Symposium Series; American Chemical Society: Washington, DC, USA, 2020; Volume 1357, pp. 161-174.

78. Liu, B.; He, L.; Wang, L.; Li, T.; Li, C.; Liu, H.; Luo, Y.; Bao, R. Protein Crystallography and Site-Direct Mutagenesis Analysis of the Poly(Ethylene Terephthalate) Hydrolase PETase from Ideonella Sakaiensis. ChemBioChem 2018, 19, 1471-1475. [CrossRef] [PubMed]

79. Fecker, T.; Galaz-Davison, P.; Engelberger, F.; Narui, Y.; Sotomayor, M.; Parra, L.P.; Ramírez-Sarmiento, C.A. Active Site Flexibility as a Hallmark for Efficient PET Degradation by I. Sakaiensis PETase. Biophys. J. 2018, 114, 1302-1312. [CrossRef] 
80. Palm, G.J.; Reisky, L.; Böttcher, D.; Müller, H.; Michels, E.A.P.; Walczak, M.C.; Berndt, L.; Weiss, M.S.; Bornscheuer, U.T.; Weber, G. Structure of the Plastic-Degrading Ideonella Sakaiensis MHETase Bound to a Substrate. Nat. Commun. 2019, 10, 1717. [CrossRef]

81. Son, H.F.; Cho, I.J.; Joo, S.; Seo, H.; Sagong, H.-Y.; Choi, S.Y.; Lee, S.Y.; Kim, K.-J. Rational Protein Engineering of Thermo-Stable PETase from Ideonella Sakaiensis for Highly Efficient PET Degradation. ACS Catal. 2019, 9, 3519-3526. [CrossRef]

82. Cui, Y.; Chen, Y.; Liu, X.; Dong, S.; Tian, Y.; Qiao, Y.; Mitra, R.; Han, J.; Li, C.; Han, X.; et al. Computational Redesign of a PETase for Plastic Biodegradation by the GRAPE Strategy. bioRxiv 2019, 787069. [CrossRef]

83. Son, H.F.; Joo, S.; Seo, H.; Sagong, H.Y.; Lee, S.H.; Hong, H.; Kim, K.J. Structural Bioinformatics-Based Protein Engineering of Thermo-Stable PETase from Ideonella Sakaiensis. Enzym. Microb. Technol. 2020, 141, 109656. [CrossRef]

84. Campo, E.A. Thermal Properties of Polymeric Materials. In Selection of Polymeric Materials; Campo, E.A., Ed.; Plastics Design Library; William Andrew Publishing: Norwich, NY, USA, 2008; pp. 103-140.

85. Pohl, F.; Eggenhuisen, J.T.; Kane, I.A.; Clare, M.A. Transport and Burial of Microplastics in Deep-Marine Sediments by Turbidity Currents. Environ. Sci. Technol. 2020, 54, 4180-4189. [CrossRef]

86. Chen, Z.; Wang, Y.; Cheng, Y.; Wang, X.; Tong, S.; Yang, H.; Wang, Z. Efficient Biodegradation of Highly Crystallized Polyethylene Terephthalate through Cell Surface Display of Bacterial PETase. Sci. Total Environ. 2020, 709, 136138. [CrossRef] [PubMed]

87. Moog, D.; Schmitt, J.; Senger, J.; Zarzycki, J.; Rexer, K.-H.; Linne, U.; Erb, T.; Maier, U.G. Using a Marine Microalga as a Chassis for Polyethylene Terephthalate (PET) Degradation. Microb. Cell Fact. 2019, 18, 1-15. [CrossRef]

88. Kim, J.W.; Park, S.-B.; Tran, Q.-G.; Cho, D.-H.; Choi, D.-Y.; Lee, Y.J.; Kim, H.-S. Functional Expression of Polyethylene TerephthalateDegrading Enzyme (PETase) in Green Microalgae. Microb. Cell Fact. 2020, 19, 97. [CrossRef] [PubMed]

89. Knott, B.C.; Erickson, E.; Allen, M.D.; Gado, J.E.; Graham, R.; Kearns, F.L.; Pardo, I.; Topuzlu, E.; Anderson, J.J.; Austin, H.P.; et al. Characterization and Engineering of a Two-Enzyme System for Plastics Depolymerization. Proc. Natl. Acad. Sci. USA 2020, 117, 25476-25485. [CrossRef]

90. Papadopoulou, A.; Hecht, K.; Buller, R. Enzymatic PET Degradation. CHIMIA Int. J. Chem. 2019, 73, 743-749. [CrossRef] [PubMed]

91. Wei, R.; Song, C.; Gräsing, D.; Schneider, T.; Bielytskyi, P.; Böttcher, D.; Matysik, J.; Bornscheuer, U.T.; Zimmermann, W. Conformational Fitting of a Flexible Oligomeric Substrate Does Not Explain the Enzymatic PET Degradation. Nat. Commun. 2019, 10, 1-4. [CrossRef] [PubMed]

92. Seo, H.; Cho, I.J.; Joo, S.; Son, H.F.; Sagong, H.-Y.; Choi, S.Y.; Lee, S.Y.; Kim, K.-J. Reply to “Conformational Fitting of a Flexible Oligomeric Substrate Does Not Explain the Enzymatic PET Degradation". Nat. Commun. 2019, 10, 1-2. [CrossRef]

93. Kawai, F.; Kawabata, T.; Oda, M. Current Knowledge on Enzymatic PET Degradation and Its Possible Application to Waste Stream Management and Other Fields. Appl. Microbiol. Biotechnol. 2019, 103, 4253-4268. [CrossRef]

94. Cousin, X.; Hotelier, T.; Giles, K.; Lievin, P.; Toutant, J.-P.; Chatonnet, A. The $\alpha / \beta$ Fold Family of Proteins Database and the Cholinesterase Gene Server ESTHER. Nucleic Acids Res. 1997, 25, 143-146. [CrossRef]

95. Sagong, H.-Y.; Seo, H.; Kim, T.; Son, H.F.; Joo, S.; Lee, S.H.; Kim, S.; Woo, J.-S.; Hwang, S.Y.; Kim, K.-J. Decomposition of the PET Film by MHETase Using Exo-PETase Function. ACS Catal. 2020, 10, 4805-4812. [CrossRef]

96. Suzuki, K.; Hori, A.; Kawamoto, K.; Thangudu, R.R.; Ishida, T.; Igarashi, K.; Samejima, M.; Yamada, C.; Arakawa, T.; Wakagi, T.; et al. Crystal Structure of a Feruloyl Esterase Belonging to the Tannase Family: A Disulfide Bond near a Catalytic Triad. Proteins Struct. Funct. Bioinform. 2014, 82, 2857-2867. [CrossRef]

97. Bollinger, A.; Thies, S.; Knieps-Grünhagen, E.; Gertzen, C.; Kobus, S.; Höppner, A.; Ferrer, M.; Gohlke, H.; Smits, S.H.J.; Jaeger, K.E. A Novel Polyester Hydrolase from the Marine Bacterium Pseudomonas Aestusnigri-Structural and Functional Insights. Front. Microbiol. 2020, 11, 114. [CrossRef]

98. Sánchez, D.; Mulet, M.; Rodríguez, A.C.; David, Z.; Lalucat, J.; García-Valdés, E. Pseudomonas Aestusnigri Sp. Nov. Isolated from Crude Oil-Contaminated Intertidal Sand Samples after the Prestige Oil Spill. Syst. Appl. Microbiol. 2014, 37, 89-94. [CrossRef]

99. Molitor, R.; Bollinger, A.; Kubicki, S.; Loeschcke, A.; Jaeger, K.-E.; Thies, S. Agar Plate-Based Screening Methods for the Identification of Polyester Hydrolysis by Pseudomonas Species. Microb. Biotechnol. 2020, 13, 274-284. [CrossRef]

100. Gomila, M.; Mulet, M.; Lalucat, J.; García-Valdés, E. Draft Genome Sequence of the Marine Bacterium Pseudomonas Aestusnigri VGXO14(T). Genome Announc. 2017, 5, e00765-17. [CrossRef]

101. Bollinger, A.; Thies, S.; Katzke, N.; Jaeger, K.-E. The Biotechnological Potential of Marine Bacteria in the Novel Lineage of Pseudomonas Pertucinogena. Microb. Biotechnol. 2020, 13, 19-31. [CrossRef]

102. Lin, H.; Truhlar, D.G. QM/MM: What Have We Learned, Where Are We, and Where Do We Go from Here? Theor. Chem. Acc. 2007, 117, 185-199. [CrossRef]

103. Magalhães, R.P.; Fernandes, H.S.; Sousa, S.F. Modelling Enzymatic Mechanisms with QM/MM Approaches: Current Status and Future Challenges. Isr. J. Chem. 2020, 60, 655-666. [CrossRef]

104. Senn, H.M.; Thiel, W. QM/MM Methods for Biomolecular Systems. Angew. Chem. Int. Ed. 2009, 48, 1198-1229. [CrossRef] [PubMed]

105. Sulaiman, S.; You, D.-J.; Kanaya, E.; Koga, Y.; Kanaya, S. Crystal Structure and Thermodynamic and Kinetic Stability of Metagenome-Derived LC-Cutinase. Biochemistry 2014, 53, 1858-1869. [CrossRef]

106. Tournier, V.; Topham, C.M.; Gilles, A.; David, B.; Folgoas, C.; Moya-Leclair, E.; Kamionka, E.; Desrousseaux, M.-L.; Texier, H.; Gavalda, S.; et al. An Engineered PET Depolymerase to Break down and Recycle Plastic Bottles. Nature 2020, 580, 216-219. [CrossRef] [PubMed] 
107. Shirke, A.N.; White, C.; Englaender, J.A.; Zwarycz, A.; Butterfoss, G.L.; Linhardt, R.J.; Gross, R.A. Stabilizing Leaf and Branch Compost Cutinase (LCC) with Glycosylation: Mechanism and Effect on PET Hydrolysis. Biochemistry 2018, 57, 1190-1200. [CrossRef] [PubMed]

108. Yan, F.; Wei, R.; Cui, Q.; Bornscheuer, U.T.; Liu, Y.J. Thermophilic Whole-Cell Degradation of Polyethylene Terephthalate Using Engineered Clostridium Thermocellum. Microb. Biotechnol. 2020, 14, 374-385. [CrossRef]

109. Kleeberg, I.; Hetz, C.; Kroppenstedt, R.M.; Müller, R.J.; Deckwer, W.D. Biodegradation of Aliphatic-Aromatic Copolyesters by Thermomonospora Fusca and Other Thermophilic Compost Isolates. Appl. Environ. Microbiol. 1998, 64, 1731-1735. [CrossRef] [PubMed]

110. Chen, S.; Tong, X.; Woodard, R.W.; Du, G.; Wu, J.; Chen, J. Identification and Characterization of Bacterial Cutinase. J. Biol. Chem. 2008, 283, 25854-25862. [CrossRef]

111. Lao, G.; Ghangas, G.S.; Jung, E.D.; Wilson, D.B. DNA Sequences of Three Beta-1,4-Endoglucanase Genes from Thermomonospora Fusca. J. Bacteriol. 1991, 173, 3397-3407. [CrossRef]

112. Dresler, K.; Van Den Heuvel, J.; Müller, R.J.; Deckwer, W.D. Production of a Recombinant Polyester-Cleaving Hydrolase from Thermobifida Fusca in Escherichia Coli. Bioprocess Biosyst. Eng. 2006, 29, 169-183. [CrossRef]

113. Kleeberg, I.; Welzel, K.; VandenHeuvel, J.; Müller, R.J.; Deckwer, W.D. Characterization of a New Extracellular Hydrolase from Thermobifida Fusca Degrading Aliphatic-Aromatic Copolyesters. Biomacromolecules 2005, 6, 262-270. [CrossRef]

114. Then, J.; Wei, R.; Oeser, T.; Barth, M.; Belisário-Ferrari, M.R.; Schmidt, J.; Zimmermann, W. Ca ${ }^{2+}$ and $\mathrm{Mg}^{2+}$ Binding Site Engineering Increases the Degradation of Polyethylene Terephthalate Films by Polyester Hydrolases from Thermobifida Fusca. Biotechnol. J. 2015, 10, 592-598. [CrossRef] [PubMed]

115. Dong, Q.; Yuan, S.; Wu, L.; Su, L.; Zhao, Q.; Wu, J.; Huang, W.; Zhou, J. Structure-Guided Engineering of a Thermobifida Fusca Cutinase for Enhanced Hydrolysis on Natural Polyester Substrate. Bioresour. Bioprocess. 2020, 7, 37. [CrossRef]

116. Alisch-Mark, M.; Herrmann, A.; Zimmermann, W. Increase of the Hydrophilicity of Polyethylene Terephthalate Fibres by Hydrolases from Thermomonospora Fusca and Fusarium Solani f. Sp. Pisi. Biotechnol. Lett. 2006, 28, 681-685. [CrossRef] [PubMed]

117. Su, L.; Woodard, R.W.; Chen, J.; Wu, J. Extracellular Location of Thermobifida Fusca Cutinase Expressed in Escherichia Coli BL21(DE3) without Mediation of a Signal Peptide. Appl. Environ. Microbiol. 2013, 79, 4192-4198. [CrossRef] [PubMed]

118. Yang, Y.; Malten, M.; Grote, A.; Jahn, D.; Deckwer, W.-D. Codon Optimized Thermobifida Fusca Hydrolase Secreted by Bacillus Megaterium. J. Anat. 2006, 189 Pt 3, 503-505.

119. Sinsereekul, N.; Wangkam, T.; Thamchaipenet, A.; Srikhirin, T.; Eurwilaichitr, L.; Champreda, V. Recombinant Expression of BTA Hydrolase in Streptomyces Rimosus and Catalytic Analysis on Polyesters by Surface Plasmon Resonance. Appl. Microbiol. Biotechnol. 2010, 86, 1775-1784. [CrossRef]

120. Sousa, S.F.; Ribeiro, A.J.M.; Coimbra, J.T.S.; Neves, R.P.P.; Martins, S.A.; Moorthy, N.S.H.N.; Fernandes, P.A.; Ramos, M.J. Protein-Ligand Docking in the New Millennium-A Retrospective of 10 Years in the Field. Curr. Med. Chem. 2013, 20, 2296-2314. [CrossRef]

121. Sousa, S.F.; Fernandes, P.A.; Ramos, M.J. Protein-Ligand Docking: Current Status and Future Challenges. Proteins Struct. Funct. Bioinform. 2006, 65, 15-26. [CrossRef]

122. Kitchen, D.B.; Decornez, H.; Furr, J.R.; Bajorath, J. Docking and scoring in virtual screening for drug discovery: Methods and applications. Nat. Rev. Drug Discov. 2004, 3, 935-949. [CrossRef]

123. Halperin, I.; Ma, B.; Wolfson, H.; Nussinov, R. Principles of Docking: An Overview of Search Algorithms and a Guide to Scoring Functions. Proteins: Struct. Funct. Bioinform. 2002, 443, 409-443. [CrossRef]

124. Ferreira, L.G.; Santos, R.N.; Oliva, G.; Andricopulo, A.D. Molecular Docking and Structure-Based Drug Design Strategies. Molecules 2015, 20, 13384-13421. [CrossRef] [PubMed]

125. Serrano, C.; Teixeira, C.S.S.; Cooper, D.N.; Carneiro, J.; Lopes-Marques, M.; Stenson, P.D.; Amorim, A.; Prata, M.J.; Sousa, S.F.; Azevedo, L. Compensatory Epistasis Explored by Molecular Dynamics Simulations. Hum. Genet. 2021, 140, 1329-1342. [CrossRef] [PubMed]

126. Hospital, A.; Goñi, J.R.; Orozco, M.; Gelpí, J.L. Molecular Dynamics Simulations: Advances and Applications. Adv. Appl. Bioinform. Chem. 2015, 8, 37-47. [PubMed]

127. Lazim, R.; Suh, D.; Choi, S. Advances in Molecular Dynamics Simulations and Enhanced Sampling Methods for the Study of Protein Systems. Int. J. Mol. Sci. 2020, 21, 6339. [CrossRef] [PubMed]

128. Karplus, M.; McCammon, J.A. Molecular Dynamics Simulations of Biomolecules. Nat. Struct. Biol. 2002, 9, 646-652. [CrossRef]

129. Klepeis, J.L.; Lindorff-Larsen, K.; Dror, R.O.; Shaw, D.E. Long-Timescale Molecular Dynamics Simulations of Protein Structure and Function. Curr. Opin. Struct. Biol. 2009, 19, 120-127. [CrossRef]

130. Hollingsworth, S.A.; Dror, R.O. Molecular Dynamics Simulation for All. Neuron 2018, 99, 1129-1143. [CrossRef]

131. Pati, A.; Sikorski, J.; Nolan, M.; Lapidus, A.; Copeland, A.; Glavina Del Rio, T.; Lucas, S.; Chen, F.; Tice, H.; Pitluck, S.; et al. Complete Genome Sequence of Saccharomonospora Viridis Type Strain (P101T). Stand. Genom. Sci. 2009, 1, 141-149. [CrossRef]

132. Miyakawa, T.; Mizushima, H.; Ohtsuka, J.; Oda, M.; Kawai, F.; Tanokura, M. Structural Basis for the Ca ${ }^{2+}$-Enhanced Thermostability and Activity of PET-Degrading Cutinase-like Enzyme from Saccharomonospora Viridis AHK190. Appl. Microbiol. Biotechnol. 2015, 99, 4297-4307. [CrossRef] 
133. Numoto, N.; Kamiya, N.; Bekker, G.-J.; Yamagami, Y.; Inaba, S.; Ishii, K.; Uchiyama, S.; Kawai, F.; Ito, N.; Oda, M. Structural Dynamics of the PET-Degrading Cutinase-like Enzyme from Saccharomonospora Viridis AHK190 in Substrate-Bound States Elucidates the $\mathrm{Ca}^{2+}$-Driven Catalytic Cycle. Biochemistry 2018, 57, 5289-5300. [CrossRef]

134. Oda, M.; Yamagami, Y.; Inaba, S.; Oida, T.; Yamamoto, M.; Kitajima, S.; Kawai, F. Enzymatic Hydrolysis of PET: Functional Roles of Three $\mathrm{Ca}^{2+}$ Ions Bound to a Cutinase-like Enzyme, Cut190*, and Its Engineering for Improved Activity. Appl. Microbiol. Biotechnol. 2018, 102, 10067-10077. [CrossRef]

135. Senga, A.; Numoto, N.; Yamashita, M.; Iida, A.; Ito, N.; Kawai, F.; Oda, M. Multiple Structural States of Ca ${ }^{2+}-$ Regulated PET Hydrolase, Cut190, and Its Correlation with Activity and Stability. J. Biochem. 2021, 169, 207-213. [CrossRef]

136. Emori, M.; Numoto, N.; Senga, A.; Bekker, G.-J.; Kamiya, N.; Kobayashi, Y.; Ito, N.; Kawai, F.; Oda, M. Structural Basis of Mutants of PET-Degrading Enzyme from Saccharomonospora Viridis AHK190 with High Activity and Thermal Stability. Proteins Struct. Funct. Bioinform. 2021, 89, 502-511. [CrossRef] [PubMed]

137. Kawabata, T.; Oda, M.; Kawai, F. Mutational Analysis of Cutinase-like Enzyme, Cut190, Based on the 3D Docking Structure with Model Compounds of Polyethylene Terephthalate. J. Biosci. Bioeng. 2017, 124, 28-35. [CrossRef]

138. Hantani, Y.; Imamura, H.; Yamamoto, T.; Senga, A.; Yamagami, Y.; Kato, M.; Kawai, F.; Oda, M. Functional Characterizations of Polyethylene Terephthalate-Degrading Cutinase-like Enzyme Cut190 Mutants Using Bis(2-Hydroxyethyl) Terephthalate as the Model Substrate. AIMS Biophys. 2018, 5, 290-302. [CrossRef]

139. Herrero Acero, E.; Ribitsch, D.; Steinkellner, G.; Gruber, K.; Greimel, K.; Eiteljoerg, I.; Trotscha, E.; Wei, R.; Zimmermann, W.; Zinn, M.; et al. Enzymatic Surface Hydrolysis of PET: Effect of Structural Diversity on Kinetic Properties of Cutinases from Thermobifida. Macromolecules 2011, 44, 4632-4640. [CrossRef]

140. Ribitsch, D.; Acero, E.H.; Greimel, K.; Eiteljoerg, I.; Trotscha, E.; Freddi, G.; Schwab, H.; Guebitz, G.M. Characterization of a New Cutinase from Thermobifida Alba for PET-Surface Hydrolysis. Biocatal. Biotransformation 2012, 30, 2-9. [CrossRef]

141. Roth, C.; Wei, R.; Oeser, T.; Then, J.; Föllner, C.; Zimmermann, W.; Sträter, N. Structural and Functional Studies on a Thermostable Polyethylene Terephthalate Degrading Hydrolase from Thermobifida Fusca. Appl. Microbiol. Biotechnol. 2014, 98, 7815-7823. [CrossRef] [PubMed]

142. Ribitsch, D.; Hromic, A.; Zitzenbacher, S.; Zartl, B.; Gamerith, C.; Pellis, A.; Jungbauer, A.; Lyskowski, A.; Steinkellner, G.; Gruber, K.; et al. Small Cause, Large Effect: Structural Characterization of Cutinases from Thermobifida Cellulosilytica. Biotechnol. Bioeng. 2017, 114, 2481-2488. [CrossRef]

143. Gamerith, C.; Zartl, B.; Pellis, A.; Guillamot, F.; Marty, A.; Acero, E.H.; Guebitz, G.M. Enzymatic Recovery of Polyester Building Blocks from Polymer Blends. Process Biochem. 2017, 59, 58-64. [CrossRef]

144. Barth, M.; Oeser, T.; Wei, R.; Then, J.; Schmidt, J.; Zimmermann, W. Effect of Hydrolysis Products on the Enzymatic Degradation of Polyethylene Terephthalate Nanoparticles by a Polyester Hydrolase from Thermobifida Fusca. Biochem. Eng. J. 2015, 93, 222-228. [CrossRef]

145. Wei, R.; Oeser, T.; Schmidt, J.; Meier, R.; Barth, M.; Then, J.; Zimmermann, W. Engineered Bacterial Polyester Hydrolases Efficiently Degrade Polyethylene Terephthalate Due to Relieved Product Inhibition. Biotechnol. Bioeng. 2016, 113, 1658-1665. [CrossRef]

146. Barth, M.; Wei, R.; Oeser, T.; Then, J.; Schmidt, J.; Wohlgemuth, F.; Zimmermann, W. Enzymatic Hydrolysis of Polyethylene Terephthalate Films in an Ultrafiltration Membrane Reactor. J. Memb. Sci. 2015, 494, 182-187. [CrossRef]

147. Then, J.; Wei, R.; Oeser, T.; Gerdts, A.; Schmidt, J.; Barth, M.; Zimmermann, W. A Disulfide Bridge in the Calcium Binding Site of a Polyester Hydrolase Increases Its Thermal Stability and Activity against Polyethylene Terephthalate. FEBS Open Bio 2016, 6, 425-432. [CrossRef]

148. Herrero Acero, E.; Ribitsch, D.; Dellacher, A.; Zitzenbacher, S.; Marold, A.; Steinkellner, G.; Gruber, K.; Schwab, H.; Guebitz, G.M. Surface Engineering of a Cutinase from Thermobifida Cellulosilytica for Improved Polyester Hydrolysis. Biotechnol. Bioeng. 2013, 110, 2581-2590. [CrossRef] [PubMed]

149. Ribitsch, D.; Yebra, A.O.; Zitzenbacher, S.; Wu, J.; Nowitsch, S.; Steinkellner, G.; Greimel, K.; Doliska, A.; Oberdorfer, G.; Gruber, C.C.; et al. Fusion of Binding Domains to Thermobifida Cellulosilytica Cutinase to Tune Sorption Characteristics and Enhancing PET Hydrolysis. Biomacromolecules 2013, 14, 1769-1776. [CrossRef]

150. Ribitsch, D.; Herrero Acero, E.; Przylucka, A.; Zitzenbacher, S.; Marold, A.; Gamerith, C.; Tscheließnig, R.; Jungbauer, A.; Rennhofer, H.; Lichtenegger, H.; et al. Enhanced Cutinase-Catalyzed Hydrolysis of Polyethylene Terephthalate by Covalent Fusion to Hydrophobins. Appl. Environ. Microbiol. 2015, 81, 3586-3592. [CrossRef]

151. Pellis, A.; Gamerith, C.; Ghazaryan, G.; Ortner, A.; Herrero Acero, E.; Guebitz, G.M. Ultrasound-Enhanced Enzymatic Hydrolysis of Poly(Ethylene Terephthalate). Bioresour. Technol. 2016, 218, 1298-1302. [CrossRef] [PubMed]

152. Gott, K.P.; Burgess, L.W.; Balmas, V.; Duff, J. Mycogeography of Fusarium: Fusarium Species in Soils from Palm Valley, Central Australia. Australas. Plant Pathol. 1994, 23, 112-117. [CrossRef]

153. Dimarogona, M.; Nikolaivits, E.; Kanelli, M.; Christakopoulos, P.; Sandgren, M.; Topakas, E. Structural and Functional Studies of a Fusarium Oxysporum Cutinase with Polyethylene Terephthalate Modification Potential. Biochim. Biophys. Acta 2015, 1850, 2308-2317. [CrossRef]

154. Nikolaivits, E.; Kokkinou, A.; Karpusas, M.; Topakas, E. Microbial Host Selection and Periplasmic Folding in Escherichia Coli Affect the Biochemical Characteristics of a Cutinase from Fusarium Oxysporum. Protein Expr. Purif. 2016, 127, 1-7. [CrossRef]

155. Kanelli, M.; Vasilakos, S.; Nikolaivits, E.; Ladas, S.; Christakopoulos, P.; Topakas, E. Surface Modification of Poly(Ethylene Terephthalate) (PET) Fibers by a Cutinase from Fusarium Oxysporum. Process Biochem. 2015, 50, 1885-1892. [CrossRef] 
156. Maheshwari, R.; Bharadwaj, G.; Bhat, M.K. Thermophilic Fungi: Their Physiology and Enzymes. Microbiol. Mol. Biol. Rev. 2000, 64, 461-488. [CrossRef]

157. Ronkvist, Å.M.; Xie, W.; Lu, W.; Gross, R.A. Cutinase-Catalyzed Hydrolysis of Poly(Ethylene Terephthalate). Macromolecules 2009, 42, 5128-5138. [CrossRef]

158. de Castro, A.M.; Carniel, A.; Nicomedes Junior, J.; da Conceição Gomes, A.; Valoni, É. Screening of Commercial Enzymes for Poly(Ethylene Terephthalate) (PET) Hydrolysis and Synergy Studies on Different Substrate Sources. J. Ind. Microbiol. Biotechnol. 2017, 44, 835-844. [CrossRef] [PubMed]

159. Kold, D.; Dauter, Z.; Laustsen, A.K.; Brzozowski, A.M.; Turkenburg, J.P.; Nielsen, A.D.; Koldsø, H.; Petersen, E.; Schiøtt, B.; De Maria, L.; et al. Thermodynamic and Structural Investigation of the Specific SDS Binding of Humicola Insolens Cutinase. Protein Sci. 2014, 23, 1023-1035. [CrossRef] [PubMed]

160. Kazenwadel, C.; Eiben, S.; Maurer, S.; Beuttler, H.; Wetzl, D.; Hauer, B.; Koschorreck, K. Thiol-Functionalization of Acrylic Ester Monomers Catalyzed by Immobilized Humicola Insolens Cutinase. Enzym. Microb. Technol. 2012, 51, 9-15. [CrossRef]

161. UniProt: The Universal Protein Knowledgebase in 2021. Nucleic Acids Res. 2021, 49, D480-D489. [CrossRef] [PubMed]

162. Kobayashi, S.; Makino, A. Enzymatic Polymer Synthesis: An Opportunity for Green Polymer Chemistry. Chem. Rev. 2009, 109, 5288-5353. [CrossRef]

163. Fabbri, F.; Bertolini, F.A.; Guebitz, G.M.; Pellis, A. Biocatalyzed Synthesis of Flavor Esters and Polyesters: A Design of Experiments (DoE) Approach. Int. J. Mol. Sci. 2021, 22, 8493. [CrossRef] [PubMed]

164. Feder, D.; Gross, R.A. Exploring Chain Length Selectivity in HIC-Catalyzed Polycondensation Reactions. Biomacromolecules 2010, 11, 690-697. [CrossRef] [PubMed]

165. Carniel, A.; Valoni, É.; Nicomedes, J.; da Conceição Gomes, A.; de Castro, A.M. Lipase from Candida Antarctica (CALB) and Cutinase from Humicola Insolens Act Synergistically for PET Hydrolysis to Terephthalic Acid. Process Biochem. 2017, 59, 84-90. [CrossRef]

166. Greimel, K.; Marold, A.; Sohar, C.; Feola, R.; Temel, A.; Schoenbacher, T.; Herrero Acero, E.; Guebitz, G.M. Enzymatic Hydrolysis of Polyester Based Coatings. React. Funct. Polym. 2013, 73, 1335-1339. [CrossRef]

167. Quartinello, F.; Vajnhandl, S.; Volmajer Valh, J.; Farmer, T.J.; Vončina, B.; Lobnik, A.; Herrero Acero, E.; Pellis, A.; Guebitz, G.M. Synergistic Chemo-Enzymatic Hydrolysis of Poly(Ethylene Terephthalate) from Textile Waste. Microb. Biotechnol. 2017, 10, 1376-1383. [CrossRef] [PubMed]

168. Carniel, A.; da Conceição Gomes, A.; Coelho, M.A.Z.; de Castro, A.M. Process Strategies to Improve Biocatalytic Depolymerization of Post-Consumer PET Packages in Bioreactors, and Investigation on Consumables Cost Reduction. Bioprocess Biosyst. Eng. 2021, 44, 507-516. [CrossRef] [PubMed]

169. de Queiros Eugenio, E.; Campisano, I.S.P.; de Castro, A.M.; Coelho, M.A.Z.; Langone, M.A.P. Experimental and Mathematical Modeling Approaches for Biocatalytic Post-Consumer Poly(Ethylene Terephthalate) Hydrolysis. J. Biotechnol. 2021, 341 , 76-85. [CrossRef] [PubMed]

170. Quartinello, F.; Kremser, K.; Vecchiato, S.; Schoen, H.; Vielnascher, R.; Ploszczanski, L.; Pellis, A.; Guebitz, G.M. Increased Flame Retardancy of Enzymatic Functionalized PET and Nylon Fabrics via DNA Immobilization. Front. Chem. 2019, 7, 685. [CrossRef] [PubMed]

171. de Castro, A.M.; Carniel, A.; Stahelin, D.; Chinelatto Junior, L.S.; de Angeli Honorato, H.; de Menezes, S.M.C. High-Fold Improvement of Assorted Post-Consumer Poly(Ethylene Terephthalate) (PET) Packages Hydrolysis Using Humicola Insolens Cutinase as a Single Biocatalyst. Process Biochem. 2019, 81, 85-91. [CrossRef]

172. Su, L.; Hong, R.; Kong, D.; Wu, J. Enhanced Activity towards Polyacrylates and Poly(Vinyl Acetate) by Site-Directed Mutagenesis of Humicola Insolens Cutinase. Int. J. Biol. Macromol. 2020, 162, 1752-1759. [CrossRef] [PubMed]

173. Shirke, A.N.; Butterfoss, G.L.; Saikia, R.; Basu, A.; de Maria, L.; Svendsen, A.; Gross, R.A. Engineered Humicola Insolens Cutinase for Efficient Cellulose Acetate Deacetylation. Biotechnol. J. 2017, 12, 1700188. [CrossRef]

174. Martinez, C.; De Geus, P.; Lauwereys, M.; Matthyssens, G.; Cambillau, C. Fusarium Solani Cutinase Is a Lipolytic Enzyme with a Catalytic Serine Accessible to Solvent. Nature 1992, 356, 615-618. [CrossRef]

175. Summerell, B.A.; Laurence, M.H.; Liew, E.C.Y.; Leslie, J.F. Biogeography and Phylogeography of Fusarium: A Review. Fungal Divers. 2010, 44, 3-13. [CrossRef]

176. LIN, T.-S.; KOLATTUKUDY, P.E. Structural Studies on Cutinase, a Glycoprotein Containing Novel Amino Acids and Glucuronic Acid Amide at the N Terminus. Eur. J. Biochem. 1980, 106, 341-351. [CrossRef]

177. Martinez, C.; Nicolas, A.; van Tilbeurgh, H.; Egloff, M.P.; Cambillau, C.; Cudrey, C.; Verger, R. Cutinase, a Lipolytic Enzyme with a Preformed Oxyanion Hole. Biochemistry 1994, 33, 83-89. [CrossRef] [PubMed]

178. Nicolas, A.; Egmond, M.; Verrips, C.T.; De Vlieg, J.; Longhi, S.; Cambillau, C.; Martinez, C. Contribution of Cutinase Serine 42 Side Chain to the Stabilization of the Oxyanion Transition State. Biochemistry 1996, 35, 398-410. [CrossRef] [PubMed]

179. Longhi, S.; Nicolas, A.; Creveld, L.; Egmond, M.; Verrips, C.T.; De Vlieg, J.; Martinez, C.; Cambillau, C. Dynamics of Fusarium Solani Cutinase Investigated through Structural Comparison among Different Crystal Forms of Its Variants. Proteins Struct. Funct. Genet. 1996, 26, 442-458. [CrossRef]

180. Longhi, S.; Mannesse, M.; Verheij, H.M.; De Haas, G.H.; Egmond, M.; Knoops-Mouthuy, E.; Cambillau, C. Crystal Structure of Cutinase Covalently Inhibited by a Triglyceride Analogue. Protein Sci. 1997, 6, 275-286. [CrossRef] 
181. Longhi, S.; Czjzek, M.; Lamzin, V.; Nicolas, A.; Cambillau, C. Atomic Resolution (1.0 ̊̊) Crystal Structure of Fusarium Solani Cutinase: Stereochemical Analysis11Edited by R. Huber. J. Mol. Biol. 1997, 268, 779-799. [CrossRef] [PubMed]

182. Rutten, L.; Wieczorek, B.; Mannie, J.-P.B.A.; Kruithof, C.A.; Dijkstra, H.P.; Egmond, M.R.; Lutz, M.; Klein Gebbink, R.J.M.; Gros, P.; van Koten, G. Solid-State Structural Characterization of Cutinase-ECE-Pincer-Metal Hybrids. Chem.-A Eur. J. 2009, 15, 4270-4280. [CrossRef] [PubMed]

183. Heumann, S.; Eberl, A.; Pobeheim, H.; Liebminger, S.; Fischer-Colbrie, G.; Almansa, E.; Cavaco-Paulo, A.; Gübitz, G.M. New Model Substrates for Enzymes Hydrolysing Polyethyleneterephthalate and Polyamide Fibres. J. Biochem. Biophys. Methods 2006, 69, 89-99. [CrossRef] [PubMed]

184. Araújo, R.; Silva, C.; O’Neill, A.; Micaelo, N.; Guebitz, G.; Soares, C.M.; Casal, M.; Cavaco-Paulo, A. Tailoring Cutinase Activity towards Polyethylene Terephthalate and Polyamide 6,6 Fibers. J. Biotechnol. 2007, 128, 849-857. [CrossRef]

185. Eberl, A.; Heumann, S.; Brückner, T.; Araujo, R.; Cavaco-Paulo, A.; Kaufmann, F.; Kroutil, W.; Guebitz, G.M. Enzymatic Surface Hydrolysis of Poly(Ethylene Terephthalate) and Bis(Benzoyloxyethyl) Terephthalate by Lipase and Cutinase in the Presence of Surface Active Molecules. J. Biotechnol. 2009, 143, 207-212. [CrossRef] [PubMed]

186. Goto, S.; Sugiyama, J.; Iizuka, H. A Taxonomic Study of Antarctic Yeasts. Mycologia 1969, 61, 748-774. [CrossRef] [PubMed]

187. Heldt-Hansen, H.P.; Ishii, M.; Patkar, S.A.; Hansen, T.T.; Eigtved, P. A New Immobilized Positional Nonspecific Lipase for Fat Modification and Ester Synthesis. In Biocatalysis in Agricultural Biotechnology; ACS Symposium Series; American Chemical Society: Washington, DC, USA, 1989; Volume 389, pp. 11-158.

188. Uppenberg, J.; Ohmer, N.; Norin, M.; Hult, K.; Kleywegt, G.J.; Patkar, S.; Waagen, V.; Anthonsen, T.; Jones, T.A. Crystallographic and Molecular-Modeling Studies of Lipase B from Candida Antarctica Reveal a Stereospecificity Pocket for Secondary Alcohols. Biochemistry 1995, 34, 16838-16851. [CrossRef] [PubMed]

189. Strzelczyk, P.; Bujacz, G.D.; Kiełbasiński, P.; Błaszczyk, J. Crystal and Molecular Structure of Hexagonal Form of Lipase B from Candida Antarctica. Acta Biochim. Pol. 2016, 63, 103-109. [CrossRef] [PubMed]

190. Anderson, E.M.; Larsson, K.M.; Kirk, O. One Biocatalyst-Many Applications: The Use of Candida Antarctica B-Lipase in Organic Synthesis. Biocatal. Biotransformation 1998, 16, 181-204. [CrossRef]

191. Stauch, B.; Fisher, S.J.; Cianci, M. Open and Closed States of Candida Antarctica Lipase B: Protonation and the Mechanism of Interfacial Activation. J. Lipid Res. 2015, 56, 2348-2358. [CrossRef]

192. The Sequence, Crystal Structure Determination and Refinement of Two Crystal Forms of Lipase B from Candida Antarctica. Int. J. Biol. Macromol. 1994, 140, 761-770.

193. Park, A.; Kim, S.; Park, J.; Joe, S.; Min, B.; Oh, J.; Song, J.; Park, S.Y.; Park, S.; Lee, H. Structural and Experimental Evidence for the Enantiomeric Recognition toward a Bulky Sec-Alcohol by Candida Antarctica Lipase B. ACS Catal. 2016, 6, 7458-7465. [CrossRef]

194. Qian, Z.; Horton, J.R.; Cheng, X.; Lutz, S. Structural Redesign of Lipase B from Candida Antarctica by Circular Permutation and Incremental Truncation. J. Mol. Biol. 2009, 393, 191-201. [CrossRef]

195. Xie, Y.; An, J.; Yang, G.; Wu, G.; Zhang, Y.; Cui, L.; Feng, Y. Enhanced Enzyme Kinetic Stability by Increasing Rigidity within the Active Site. J. Biol. Chem. 2014, 289, 7994-8006. [CrossRef] [PubMed]

196. Cen, Y.; Singh, W.; Arkin, M.; Moody, T.S.; Huang, M.; Zhou, J.; Wu, Q.; Reetz, M.T. Artificial Cysteine-Lipases with High Activity and Altered Catalytic Mechanism Created by Laboratory Evolution. Nat. Commun. 2019, 10, 3198. [CrossRef] [PubMed]

197. Xu, J.; Cen, Y.; Singh, W.; Fan, J.; Wu, L.; Lin, X.; Zhou, J.; Huang, M.; Reetz, M.T.; Wu, Q. Stereodivergent Protein Engineering of a Lipase to Access All Possible Stereoisomers of Chiral Esters with Two Stereocenters. J. Am. Chem. Soc. 2019, 141, 7934-7945. [CrossRef]

198. Silvestrini, L.; Cianci, M. Principles of Lipid-Enzyme Interactions in the Limbus Region of the Catalytic Site of Candida Antarctica Lipase B. Int. J. Biol. Macromol. 2020, 158, 358-363. [CrossRef] [PubMed]

199. Korpecka, J.; Heumann, S.; Billig, S.; Zimmermann, W.; Zinn, M.; Ihssen, J.; Cavaco-Paulo, A.; Guebitz, G.M. Hydrolysis of Cutin by PET-Hydrolases. Macromol. Symp. 2010, 296, 342-346. [CrossRef]

200. Vertommen, M.; Nierstrasz, V.A.; van der Veer, M.; Warmoeskerken, M.M. Enzymatic Surface Modification of Poly(Ethylene Terephthalate). J. Biotechnol. 2005, 120, 376-386. [CrossRef] [PubMed]

201. Hong, S.Y.; Yoo, Y.J. Activity Enhancement of Candida Antarctica Lipase B by Flexibility Modulation in Helix Region Surrounding the Active Site. Appl. Biochem. Biotechnol. 2013, 170, 925-933. [CrossRef]

202. Park, H.J.; Park, K.; Kim, Y.H.; Yoo, Y.J. Computational Approach for Designing Thermostable Candida Antarctica Lipase B by Molecular Dynamics Simulation. J. Biotechnol. 2014, 192, 66-70. [CrossRef]

203. Le, Q.A.T.; Joo, J.C.; Yoo, Y.J.; Kim, Y.H. Development of Thermostable Candida Antarctica Lipase B through Novel in Silico Design of Disulfide Bridge. Biotechnol. Bioeng. 2012, 109, 867-876. [CrossRef]

204. Suen, W.; Zhang, N.; Xiao, L.; Madison, V.; Zaks, A. Improved Activity and Thermostability of Candida Antarctica Lipase B by DNA Family Shuffling. Protein Eng. Des. Sel. 2004, 17, 133-140. [CrossRef]

205. Zhang, N.; Suen, W.; Windsor, W.; Xiao, L.; Madison, V.; Zaks, A. Improving Tolerance of Candida Antarctica Lipase B towards Irreversible Thermal Inactivation through Directed Evolution. Protein Eng. Des. Sel. 2003, 16, 599-605. [CrossRef]

206. Rotticci, D.; Rotticci-Mulder, J.C.; Denman, S.; Norin, T.; Hult, K. Improved Enantioselectivity of a Lipase by Rational Protein Engineering. ChemBioChem 2001, 2, 766-770. [CrossRef]

207. Magnusson, A.O.; Rotticci-Mulder, J.C.; Santagostino, A.; Hult, K. Creating Space for Large Secondary Alcohols by Rational Redesign of Candida Antarctica Lipase B. ChemBioChem 2005, 6, 1051-1056. [CrossRef] [PubMed] 
208. Idris, A.; Bukhari, A. Immobilized Candida Antarctica Lipase B: Hydration, Stripping off and Application in Ring Opening Polyester Synthesis. Biotechnol. Adv. 2012, 30, 550-563. [CrossRef] [PubMed]

209. Maniar, D.; Jiang, Y.; Woortman, A.J.J.; van Dijken, J.; Loos, K. Furan-Based Copolyesters from Renewable Resources: Enzymatic Synthesis and Properties. ChemSusChem 2019, 12, 990-999. [CrossRef] [PubMed]

210. Thumarat, U.; Kawabata, T.; Nakajima, M.; Nakajima, H.; Sugiyama, A.; Yazaki, K.; Tada, T.; Waku, T.; Tanaka, N.; Kawai, F. Comparison of Genetic Structures and Biochemical Properties of Tandem Cutinase-Type Polyesterases from Thermobifida Alba AHK119. J. Biosci. Bioeng. 2015, 120, 491-497. [CrossRef]

211. Kitadokoro, K.; Matsui, S.; Osokoshi, R.; Nakata, K.; Kamitani, S. Expression, Purification and Crystallization of Thermostable Mutant of Cutinase Est1 from Thermobifida alba. Adv. Biosci. Biotechnol. 2018, 9, 215-223. [CrossRef]

212. Tsiklinsky, P. Sur Les Mucedinees Thermophiles. Ann. Inst. Pasteur 1899, 13, 501-505.

213. Arima, K.; Liu, W.-H.; Beppu, T. Studies on the Lipase of Thermophilic Fungus Humicola Lanuginosa. Agric. Biol. Chem. 1972, 36, 893-895. [CrossRef]

214. Chew, Y.H.; Chua, L.S.; Cheng, K.K.; Sarmidi, M.R.; Aziz, R.A.; Lee, C.T. Kinetic Study on the Hydrolysis of Palm Olein Using Immobilized Lipase. Biochem. Eng. J. 2008, 39, 516-520. [CrossRef]

215. Tangkam, K.; Weber, N.; Wiege, B. Solvent-Free Lipase-Catalyzed Preparation of Diglycerides from Co-Products of Vegetable Oil Refining. Grasas Y Aceites 2008, 59, 245-253.

216. Sivalingam, G.; Chattopadhyay, S.; Madras, G. Solvent Effects on the Lipase Catalyzed Biodegradation of Poly (??-Caprolactone) in Solution. Polym. Degrad. Stab. 2003, 79, 413-418. [CrossRef]

217. Reddy, J.R.C.; Vijeeta, T.; Karuna, M.S.L.; Rao, B.V.S.K.; Prasad, R.B.N. Lipase-Catalyzed Preparation of Palmitic and Stearic Acid-Rich Phosphatidylcholine. J. Am. Oil Chem. Soc. 2005, 82, 727-730. [CrossRef]

218. Brueckner, T.; Eberl, A.; Heumann, S.; Rabe, M.; Guebitz, G.M. Enzymatic and Chemical Hydrolysis of Poly(Ethylene Terephthalate) Fabrics. J. Polym. Sci. Part A Polym. Chem. 2008, 46, 6435-6443. [CrossRef]

219. Lawson, D.M.; Brzozowski, A.M.; Rety, S.; Verma, C.; Dodson, G.G. Probing the Nature of Substrate Binding in Humicola Lanuginosa Lipase through X-Ray Crystallography and Intuitive Modelling. Protein Eng. Des. Sel. 1994, 7, 543-550. [CrossRef]

220. Derewenda, U.; Swenson, L.; Wei, Y.; Green, R.; Kobos, P.M.; Joerger, R.; Haas, M.J.; Derewenda, Z.S. Conformational Lability of Lipases Observed in the Absence of an Oil-Water Interface: Crystallographic Studies of Enzymes from the Fungi Humicola Lanuginosa and Rhizopus Delemar. J. Lipid Res. 1994, 35, 524-534. [CrossRef]

221. Holmquist, M.; Clausen, I.G.; Patkar, S.; Svendsen, A.; Hult, K. Probing a Functional Role of Glu87 and Trp89 in the Lid OfHumicola Lanuginosa Lipase through Transesterification Reactions in Organic Solvent. J. Protein Chem. 1995, 14, $217-224$. [CrossRef] [PubMed]

222. Martinelle, M.; Holmquist, M.; Clausen, I.G.; Patkar, S.; Svendsen, A.; Hult, K. The Role of Glu87 and Trp89 in the Lid of Humicola Lanuginosa Lipase. Protein Eng. Des. Sel. 1996, 9, 519-524. [CrossRef]

223. Holmquist, M.; Martinelle, M.; Clausen, I.G.; Patkar, S.; Svendsen, A.; Hult, K. Trp89 in the Lid OfHumicola Lanuginosa Lipase Is Important for Efficient Hydrolysis of Tributyrin. Lipids 1994, 29, 599-603. [CrossRef]

224. Brzozowski, A.M.; Savage, H.; Verma, C.S.; Turkenburg, J.P.; Lawson, D.M.; Svendsen, A.; Patkar, S. Structural Origins of the Interfacial Activation in Thermomyces (Humicola) Lanuginosa Lipase. Biochemistry 2000, 39, 15071-15082. [CrossRef] [PubMed]

225. Yapoudjian, S.; Ivanova, M.G.; Brzozowski, A.M.; Patkar, S.A.; Vind, J.; Svendsen, A.; Verger, R. Binding of Thermomyces (Humicola) Lanuginosa Lipase to the Mixed Micelles of Cis-Parinaric Acid/NaTDC. Eur. J. Biochem. 2002, 269, 1613-1621. [CrossRef] [PubMed]

226. Kumar, M.; Mukherjee, J.; Sinha, M.; Kaur, P.; Sharma, S.; Gupta, M.N.; Singh, T.P. Enhancement of Stability of a Lipase by Subjecting to Three Phase Partitioning (TPP): Structures of Native and TPP-Treated Lipase from Thermomyces Lanuginosa. Sustain. Chem. Process. 2015, 3, 14. [CrossRef]

227. Skjold-Jørgensen, J.; Vind, J.; Moroz, O.V.; Blagova, E.; Bhatia, V.K.; Svendsen, A.; Wilson, K.S.; Bjerrum, M.J. Controlled Lid-Opening in Thermomyces Lanuginosus Lipase- An Engineered Switch for Studying Lipase Function. Biochim. Biophys. Acta Proteins Proteom. 2017, 1865, 20-27. [CrossRef]

228. de Wijn, R.; Hennig, O.; Roche, J.; Engilberge, S.; Rollet, K.; Fernandez-Millan, P.; Brillet, K.; Betat, H.; Mörl, M.; Roussel, A.; et al. A Simple and Versatile Microfluidic Device for Efficient Biomacromolecule Crystallization and Structural Analysis by Serial Crystallography. IUCrJ 2019, 6, 454-464. [CrossRef]

229. McPherson, A.; Larson, S.B.; Kalasky, A. The Crystal Structures of Thermomyces (Humicola) Lanuginosa Lipase in Complex with Enzymatic Reactants. Curr. Enzym. Inhib. 2020, 16, 199-213. [CrossRef]

230. Omar, I.C.; Nishio, N.; Nagai, S. Production of a Thermostable Lipase by Humicola Lanuginosa Grown on Sorbitol-Corn Steep Liquor Medium. Agric. Biol. Chem. 1987, 51, 2145-2151. [CrossRef]

231. Peters, G.H.; Svendsen, A.; Langberg, H.; Vind, J.; Patkar, S.A.; Toxvaerd, S.; Kinnunen, P.K.J. Active Serine Involved in the Stabilization of the Active Site Loop in the Humicola Lanuginosa Lipase. Biochemistry 1998, 37, 12375-12383. [CrossRef] [PubMed]

232. Oeser, T.; Wei, R.; Baumgarten, T.; Billig, S.; Föllner, C.; Zimmermann, W. High Level Expression of a Hydrophobic Poly(Ethylene Terephthalate)-Hydrolyzing Carboxylesterase from Thermobifida Fusca KW3 in Escherichia Coli BL21(DE3). J. Biotechnol. 2010, 146, 100-104. [CrossRef] [PubMed]

233. Billig, S.; Oeser, T.; Birkemeyer, C.; Zimmermann, W. Hydrolysis of Cyclic Poly(Ethylene Terephthalate) Trimers by a Carboxylesterase from Thermobifida Fusca KW3. Appl. Microbiol. Biotechnol. 2010, 87, 1753-1764. [CrossRef] 
234. Barth, M.; Honak, A.; Oeser, T.; Wei, R.; Belisário-Ferrari, M.R.; Then, J.; Schmidt, J.; Zimmermann, W. A Dual Enzyme System Composed of a Polyester Hydrolase and a Carboxylesterase Enhances the Biocatalytic Degradation of Polyethylene Terephthalate Films. Biotechnol. J. 2016, 11, 1082-1087. [CrossRef]

235. Belisário-Ferrari, M.R.; Wei, R.; Schneider, T.; Honak, A.; Zimmermann, W. Fast Turbidimetric Assay for Analyzing the Enzymatic Hydrolysis of Polyethylene Terephthalate Model Substrates. Biotechnol. J. 2019, 14, 1800272. [CrossRef]

236. Qiu, L.; Yin, X.; Liu, T.; Zhang, H.; Chen, G.; Wu, S. Biodegradation of Bis(2-Hydroxyethyl) Terephthalate by a Newly Isolated Enterobacter Sp. HY1 and Characterization of Its Esterase Properties. J. Basic Microbiol. 2020, 60, 699-711. [CrossRef]

237. Xi, X.; Ni, K.; Hao, H.; Shang, Y.; Zhao, B.; Qian, Z. Secretory Expression in Bacillus Subtilis and Biochemical Characterization of a Highly Thermostable Polyethylene Terephthalate Hydrolase from Bacterium HR29. Enzym. Microb. Technol. 2021, $143,109715$. [CrossRef]

238. Kato, S.; Sakai, S.; Hirai, M.; Tasumi, E.; Nishizawa, M.; Suzuki, K.; Takai, K. Long-Term Cultivation and Metagenomics Reveal Ecophysiology of Previously Uncultivated Thermophiles Involved in Biogeochemical Nitrogen Cycle. Microbes Environ. 2018, 33, 107-110. [CrossRef]

239. Eggert, T.; Pencreac'h, G.; Douchet, I.; Verger, R.; Jaeger, K.-E. A Novel Extracellular Esterase from Bacillus Subtilis and Its Conversion to a Monoacylglycerol Hydrolase. Eur. J. Biochem. 2000, 267, 6459-6469. [CrossRef] [PubMed]

240. Jabloune, R.; Khalil, M.; Ben Moussa, I.E.; Simao-Beaunoir, A.-M.; Lerat, S.; Brzezinski, R.; Beaulieu, C. Enzymatic Degradation of P-Nitrophenyl Esters, Polyethylene Terephthalate, Cutin, and Suberin by Sub1, a Suberinase Encoded by the Plant Pathogen Streptomyces Scabies. Microbes Environ. 2020, 35, ME19086. [CrossRef] [PubMed]

241. Komeil, D.; Simao-Beaunoir, A.-M.; Beaulieu, C. Detection of Potential Suberinase-Encoding Genes in Streptomyces Scabiei Strains and Other Actinobacteria. Can. J. Microbiol. 2013, 59, 294-303. [CrossRef] [PubMed]

242. Lambert, C.; Léonard, N.; De Bolle, X.; Depiereux, E. ESyPred3D: Prediction of Proteins 3D Structures. Bioinformatics 2002, 18, 1250-1256. [CrossRef]

243. Yoon, M.-Y.; Kellis, J.; Poulose, A.J. Enzymatic Modification of Polyester. AATCC Rev. 2002, 2, 33-36.

244. Danso, D.; Schmeisser, C.; Chow, J.; Zimmermann, W.; Wei, R.; Leggewie, C.; Li, X.; Hazen, T.; Streit, W.R. New Insights into the Function and Global Distribution of Polyethylene Terephthalate (PET)-Degrading Bacteria and Enzymes in Marine and Terrestrial Metagenomes. Appl. Environ. Microbiol. 2018, 84, e02773-17. [CrossRef]

245. Wei, R.; Oeser, T.; Then, J.; Kühn, N.; Barth, M.; Schmidt, J.; Zimmermann, W. Functional Characterization and Structural Modeling of Synthetic Polyester-Degrading Hydrolases from Thermomonospora Curvata. AMB Express 2014, 4, 1-10. [CrossRef] [PubMed]

246. Henssen, A. Beiträge Zur Morphologie Und Systematik Der Thermophilen Actinomyceten. Arch. Mikrobiol. 1957, $26,373-414$. [CrossRef] [PubMed]

247. Zhang, Z.; Wang, Y.; Ruan, J. Reclassification of Thermomonospora and Microtetraspora. Int. J. Syst. Bacteriol. 1998, 48 Pt 2, 411-422. [CrossRef]

248. Chertkov, O.; Sikorski, J.; Nolan, M.; Lapidus, A.; Lucas, S.; Del Rio, T.G.; Tice, H.; Cheng, J.-F.; Goodwin, L.; Pitluck, S.; et al. Complete Genome Sequence of Thermomonospora Curvata Type Strain (B9). Stand. Genom. Sci. 2011, 4, 13-22. [CrossRef] [PubMed]

249. Islam, S.; Apitius, L.; Jakob, F.; Schwaneberg, U. Targeting Microplastic Particles in the Void of Diluted Suspensions. Environ. Int. 2019, 123, 428-435. [CrossRef]

250. Schmidt, J.; Wei, R.; Oeser, T.; Dedavid e Silva, L.A.; Breite, D.; Schulze, A.; Zimmermann, W. Degradation of Polyester Polyurethane by Bacterial Polyester Hydrolases. Polymers 2017, 9, 65. [CrossRef]

251. Liebminger, S.; Eberl, A.; Sousa, F.; Heumann, S.; Fischer-Colbrie, G.; Cavaco-Paulo, A.; Guebitz, G.M. Hydrolysis of PET and Bis-(Benzoyloxyethyl) Terephthalate with a New Polyesterase from Penicillium Citrinum. Biocatal. Biotransform. 2007, 25, 171-177. [CrossRef]

252. da Costa, A.M.; de Oliveira Lopes, V.R.; Vidal, L.; Nicaud, J.M.; de Castro, A.M.; Coelho, M.A.Z. Poly(Ethylene Terephthalate) (PET) Degradation by Yarrowia Lipolytica: Investigations on Cell Growth, Enzyme Production and Monomers Consumption. Process Biochem. 2020, 95, 81-90. [CrossRef]

253. Roberts, C.; Edwards, S.; Vague, M.; León-Zayas, R.; Scheffer, H.; Chan, G.; Swartz, N.A.; Mellies, J.L. Environmental Consortium Containing Pseudomonas and Bacillus Species Synergistically Degrades Polyethylene Terephthalate Plastic. MSphere 2020, 5, 1-20. [CrossRef] [PubMed]

254. Shirke, A.N.; Basore, D.; Butterfoss, G.L.; Bonneau, R.; Bystroff, C.; Gross, R.A. Toward Rational Thermostabilization of Aspergillus Oryzae Cutinase: Insights into Catalytic and Structural Stability. Proteins Struct. Funct. Bioinform. 2016, 84, 60-72. [CrossRef]

255. Zhiqiang, L.; Gosser, Y.; Baker, P.J.; Ravee, Y.; Ziying, L.; Alemu, G.; Huiguang, L.; Butterfoss, G.L.; Kong, X.P.; Gross, R.; et al. Structural and Functional Studies of Aspergillus Oryzae Cutinase: Enhanced Thermostability and Hydrolytic Activity of Synthetic Ester and Polyester Degradation. J. Am. Chem. Soc. 2009, 131, 15711-15716.

256. Almeida, E.L.; Rincón, A.F.C.; Jackson, S.A.; Dobson, A.D.W. In Silico Screening and Heterologous Expression of a Polyethylene Terephthalate Hydrolase (PETase)-Like Enzyme (SM14est) With Polycaprolactone (PCL)-Degrading Activity, From the Marine Sponge-Derived Strain Streptomyces Sp. SM14. Front. Microbiol. 2019, 10, 2187. [CrossRef] [PubMed] 
257. Huang, Y.-C.; Chen, G.-H.; Chen, Y.-F.; Chen, W.-L.; Yang, C.-H. Heterologous Expression of Thermostable Acetylxylan Esterase Gene from Thermobifida Fusca and Its Synergistic Action with Xylanase for the Production of Xylooligosaccharides. Biochem. Biophys. Res. Commun. 2010, 400, 718-723. [CrossRef] [PubMed]

258. Hu, X.; Thumarat, U.; Zhang, X.; Tang, M.; Kawai, F. Diversity of Polyester-Degrading Bacteria in Compost and Molecular Analysis of a Thermoactive Esterase from Thermobifida Alba AHK119. Appl. Microbiol. Biotechnol. 2010, 87, 771-779. [CrossRef] [PubMed]

259. Kitadokoro, K.; Thumarat, U.; Nakamura, R.; Nishimura, K.; Karatani, H.; Suzuki, H.; Kawai, F. Crystal Structure of Cutinase Est119 from Thermobifida Alba AHK119 That Can Degrade Modified Polyethylene Terephthalate at 1.76 ̊ Resolution. Polym. Degrad. Stab. 2012, 97, 771-775. [CrossRef]

260. Kitadokoro, K.; Kakara, M.; Matsui, S.; Osokoshi, R.; Thumarat, U.; Kawai, F.; Kamitani, S. Structural Insights into the Unique Polylactate-Degrading Mechanism of Thermobifida Alba Cutinase. FEBS J. 2019, 286, 2087-2098. [CrossRef] 